\title{
Nivel de concordancia de los perfiles nutricionales de Latinoamérica con las Guías Alimentarias para la Población Argentina
}

U N I V E R S I D A D NA C I O NA L D E L A P L A T A

F A C ULT A D DE CIENCIAS MÉDICAS

DEPARTAMENTO DE POSTGRADO

INSTITUTO DE DESARROLLO E INVESTIGACIONES PEDIÁTRICAS (IDIP)

"DR. FERNANDO E VITERI"

Hospital Sor María Ludovica / La Plata

Ministerio de Salud - Comisión de Investigaciones Científicas /

Provincia de Buenos Aires

MAESTRÍA EN NUTRICIÓN HUMANA

Producción Científica: Tesina

Tesista: María Victoria Tiscornia

Director: Lorena Allemandi

Codirector: Horacio F. Gonzalez 


\section{Índice}

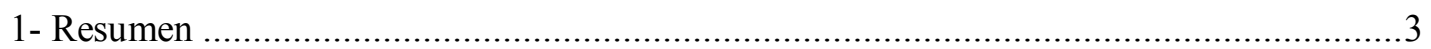

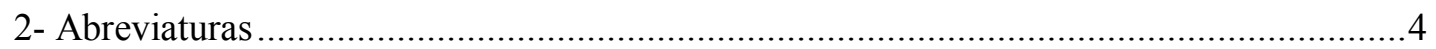

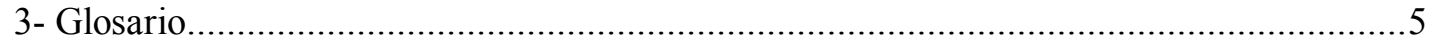

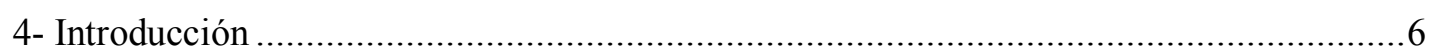

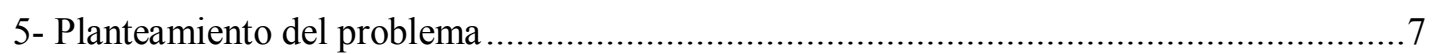

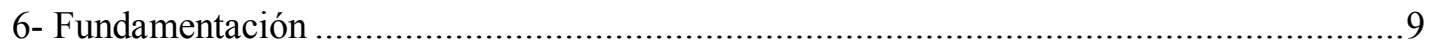

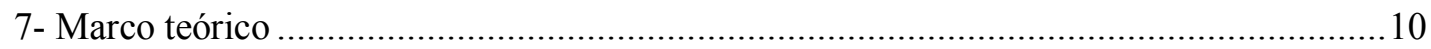

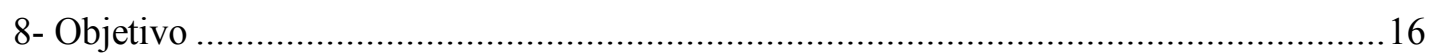

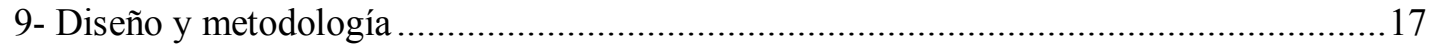

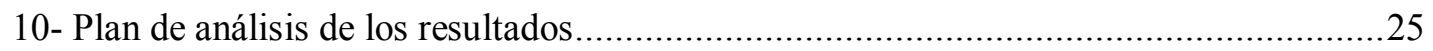

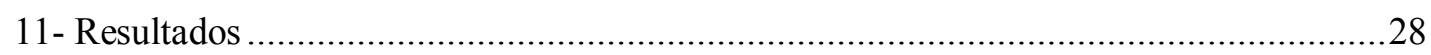

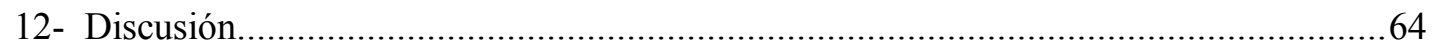

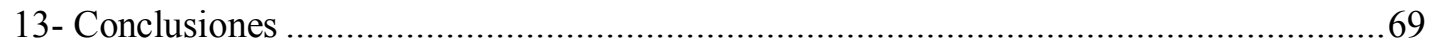

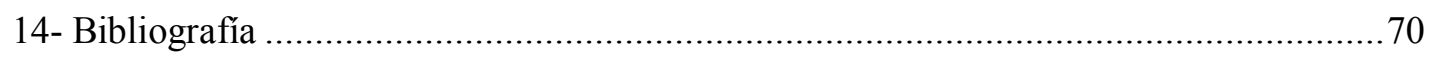

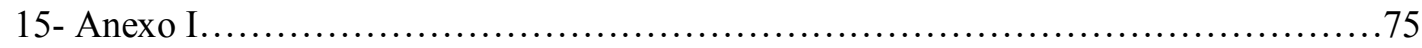

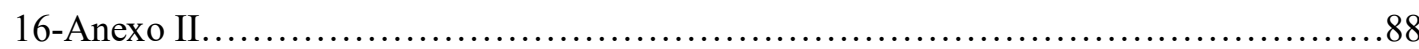

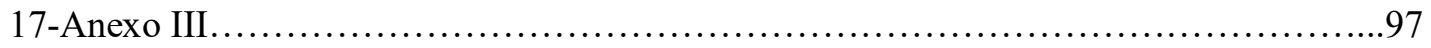




\section{1- Resumen}

Introducción: En Latinoamérica hay múltiples perfiles nutricionales utilizados en políticas de etiquetado frontal, sin embargo Argentina no cuenta con un perfil nacional. La Organización Mundial de la Salud (OMS) recomienda validar el contenido de los perfiles nutricionales tomando como referencia a las Guías Alimentarias del país en donde se aplican. De este modo, las autoridades nacionales pueden usar los perfiles nutricionales como herramienta para promover las recomendaciones alimentarias y nutricionales a través de distintas políticas alimentarias $^{1} 4556$. Objetivo Evaluar el nivel de concordancia de los perfiles nutricionales latinoamericanos con las Guías Alimentarias para la Población Argentina (GAPA). Diseño y metodología: Estudio observacional analítico de corte transversal. Se seleccionaron los perfiles nutricionales de la región utilizados en políticas de sistemas de etiquetado frontal. Se evaluó la calidad nutricional de un listado de alimentos locales según los perfiles nutricionales y se comparó con los mensajes de las GAPA. Se analizó el nivel de acuerdo con el el Índice de Concordancia, Índice Kappa y Índice Gwet's. Resultado: Se evaluaron un total de 1703 alimentos clasificados en 8 grupos y 48 categorías de alimentos. Los perfiles con mayor nivel de acuerdo con las GAPA son el perfil de la Organización Panamericana de la Salud (OPS) (Gwet's $\mathrm{AC} 1=0,663$ ), y el perfil de Uruguay (Gwet's $\mathrm{AC} 1=0,651$ ) con un acuerdo sustancial, luego el perfil de Chile (Gwet's $\mathrm{AC} 1=0,558$ ) y el perfil de Ecuador (Gwet's $\mathrm{AC} 1=0,410$ ) con un acuerdo moderado y por último el perfil de Perú (Gwet's AC1=0,373) y el perfil de Bolivia (Gwet's $\mathrm{AC} 1=0,350)$ con un acuerdo discreto. Conclusiones; Este fue el primer estudio realizado en Argentina para determinar aquellos perfiles que mejor se adaptan a las recomendaciones alimentarias-nutricionales de Argentina. Los resultados indican que la aplicación del perfil de OPS y el perfil de Uruguay en el contexto de Argentina complementan adecuadamente las GAPA a fin de ser considerados en el diseño de futuras políticas públicas para la prevención de Enfermedades No Transmisibles (ECNT).

Palabras clave: Valor Nutritivo, Enfermedad Crónica, Guías Alimentarias, Políticas Públicas de Salud, Etiquetado de Alimentos 


\section{2- Abreviaturas}

GAPA Guias Alimentarias para la Población Argentina

GABA Guias Alimentaria basadas en alimentos

ECNT Enfermedades Crónicas No Transmisibles

OMS Organización Mundial de la Salud

OPS Organización Panamericana de la Salud

FAO Organización de las Naciones Unidas para la Alimentación y la Agricultura

VD Valor Diario 


\section{3- Glosario}

Perfiles nutricionales: La ciencia de categorizar o clasificar los alimentos según su composición nutricional por razones relacionadas con la prevención de enfermedades y la promoción de la salud $^{52} 3$

Etiquetado frontal: Información que se presenta de manera gráfica en la cara frontal de los productos con el objetivo de garantizar una información veraz, simple y clara al consumidor respecto del contenido nutricional de los alimentos y bebidas no alcohólicas con el fin de mejorar la toma de decisiones en relación al consumo de alimentos. Esta información complementa la información nutricional y de ingredientes ${ }^{4}$.

Guías Alimentarias Basadas en Alimentos: Directrices basadas en evidencia científica para ayudar a las personas a lograr un patrón de alimentación saludable. Adquieren un rol trascendental como herramienta para lograr las recomendaciones nutricionales traduciendo las metas nutricionales en alimentos mediante un patrón de consumo saludable ${ }^{5}$

Ambiente obesogénico: Entorno que promueve una alta ingesta calórica y un comportamiento sedentario condicionando la presencia y el grado del sobrepeso y/o de la obesidad ${ }^{67}$. 


\section{4- Introducción}

La necesidad de diseñar e implementar políticas para la prevención de Enfermedades No Transmisibles (ECNT) ha dado lugar al desarrollo de distintos perfiles nutricionales para clasificar a los alimentos según su calidad nutricional y promover una alimentación saludable ${ }^{2}$.

De acuerdo con la Organización Mundial de la Salud (OMS), es necesario realizar una validación y comparación de los perfiles existentes para que éstos sean efectivos y adecuados ${ }^{8}$. La primera fase de validación consiste en evaluar que las clasificaciones de los perfiles nutricionales se correspondan con las recomendaciones de las Guías Alimentarias de cada país donde se aplican. De este modo, las autoridades nacionales pueden usar los perfiles nutricionales como herramienta para promover las recomendaciones alimentarias y nutricionales a través de distintas políticas alimentarias ${ }^{4} 6$

Argentina aún no cuenta con un perfil de nutrientes, lo cual representa una barrera para la implementación de políticas alimentarias como sistemas de etiquetado frontal en los envases de alimentos. Este es el primer estudio que evalúa los perfiles nutricionales latinoamericanos utilizados en políticas de etiquetado frontal en una muestra de alimentos locales y su concordancia con las Guias Alimentarias para la Población Argentina (GAPA). 


\section{5- Planteamiento del problema}

El sobrepeso, la obesidad y las ECNT asociadas se han convertido en uno de los principales problemas de salud pública en el mundo, representando más del $60 \%$ de todas las causas de muerte ${ }^{9}$. Se estima que, en 2014, 41 millones de niños menores de 5 años presentaban sobrepeso u obesidad ${ }^{7}$. La región de Latinoamérica tiene una de las prevalencias más altas, un 7,2\% de niños menores de 5 años y $58 \%$ de los adultos afectados por sobrepeso, siendo un fenómeno más extendido en mujeres ${ }^{10}$.

Argentina no es la excepción, mostrando una prevalencia en aumento en todos los grupos etarios. Según la Tercera Encuesta Nacional de Factores de Riesgo 2013 en la población mayor de 18 años la prevalencia de exceso de peso es de 57,9\%, mientras que en 2009 el valor había sido de 53,5\% y de 49,1\% en 2005 ${ }^{11}$. La Encuesta Mundial de Salud Escolar realizada en 2007 y 2012 a adolescentes de 13 a 15 años, mostró un aumento del sobrepeso del 24,5\% al 28,6\% y de la obesidad del 4,4\% al 5,9\% ${ }^{12}$. Además, los resultados de la Encuesta Nacional de Nutrición y Salud de 2004-2005 señalaron una prevalencia de obesidad del 10,4\% en niños y niñas de 6 meses a 6 años de edad ${ }^{13}$.

Una persona con sobrepeso u obesidad tiene mayor riesgo de sufrir diabetes, hipertensión, enfermedades cardiovasculares, algunos tipos de cáncer, apnea del sueño, problemas osteoarticulares, entre otros ${ }^{14}$. Cada año mueren, como mínimo, 2,8 millones de personas como resultado de la obesidad o sobrepeso ${ }^{15}$. Además tiene costos altos para el sistema de salud ${ }^{16} \mathrm{y}$ costos educacionales, laborales, ambientales, en la productividad del trabajo, edad de jubilación, autoestima, entre otros ${ }^{17}$.

En el informe sobre "Dieta, nutrición y prevención de enfermedades crónicas"18 , la OMS puso de manifiesto la relación entre la alimentación y las enfermedades crónicas de acuerdo con la evidencia científica. Este informe señala el papel potencial de una dieta rica en nutrientes como grasas totales, grasas saturadas, grasas trans, azúcares, y sodio, cuando se consumen excesivamente, en el desarrollo de las ECNT.

$\mathrm{Su}$ origen multifactorial incluye cambios en los patrones alimentarios, la desregulación del mercado, un rápido crecimiento económico, el sedentarismo, la percepción de falta de tiempo, y un mayor consumo de productos ultra-procesados altos en azúcar y grasa,entre otros ${ }^{10} 19$.

Los productos ultra-procesados son formulaciones industriales elaboradas a partir de sustancias derivadas de los alimentos o sintetizadas de otras fuentes orgánicas, con pocos alimentos enteros o ninguno. Vienen listos para consumirse o para calentar y, por lo tanto, requieren poca o ninguna preparación culinaria ${ }^{20}$. Según un informe de la Organización Panamericana de la Salud (OPS)/OMS, entre 2000 y 2013 las ventas en Latinoamérica de dichos productos aumentaron en casi $50 \%$ mientras que las ventas de las bebidas azucaradas se duplicaron. Con una venta anual de $185 \mathrm{~kg}$ per cápita de de alimentos y bebidas 
ultraprocesados, Argentina ocupa el tercer lugar en América Latina (luego de México, con 212,2 $\mathrm{kg}$ y de Chile, con 201,9 kg); y el $14^{\circ}$ lugar a nivel mundial ${ }^{20}$.

La oferta de productos procesados ricos en carbohidratos refinados, azúcares agregados, sodio y grasas saturadas tiene un impacto negativo en la salud de la población por su baja calidad nutricional y alto valor calórico. La evidencia demuestra la relación de estos nutrientes críticos con el aumento de la obesidad y las ECNT relacionadas ${ }^{21222324}$. Su consumo en forma desmedida serían la causa dietética principal del incremento del sobrepeso y las ECNT asociadas en el mundo. Dichos productos, apoyados por estrategias comerciales y publicitarias, favorecen el desplazamiento del consumo de frutas, verduras, cereales integrales y comidas caseras en respuesta a las necesidades del nuevo consumidor, principalmente de perfil urbano. La baja calidad en la alimentación actual es un problema de salud pública en todo el mundo que se hace evidente especialmente desde los años 80 cuando la proporción de este tipo de productos industrializados aumenta dentro de los sistemas y patrones alimentarios ${ }^{25}$.

Estos cambios recientes en la dieta de las poblaciones se refieren como "transición nutricional" y se encuentran asociados a un "entorno obesogénico", es decir un ambiente que influye y condiciona la presencia y el grado del sobrepeso y/o de la obesidad ${ }^{6}$.

La OMS, OPS y Organización de las Naciones Unidas para la Alimentación y la Agricultura (FAO) recomiendan a sus Estados Miembros adoptar una serie de intervenciones costoefectivas para transformar el ambiente obesogénico y hacer que el consumo de alimentos saludables y la actividad física sean la opción más fácil. Entre estas medidas se recomienda estimular la producción, la venta y el consumo de alimentos saludables, y regular la producción o desincentivar el consumo de productos ultra-procesados con alto contenido de azúcar, sal y grasas, a través de medidas fiscales, regulación de la publicidad y el etiquetado frontal de advertencia nutricional ${ }^{710}$.

La necesidad de diseñar e implementar este tipo de políticas para la prevención de ECNT, ha dado lugar al desarrollo de distintos perfiles nutricionales para clasificar a los alimentos según su calidad nutricional y promover una alimentación saludable. La OMS define a los perfiles como "la ciencia de clasificar o categorizar los alimentos de acuerdo con su composición nutricional por razones relacionadas con la prevención de enfermedades o la promoción de la salud"26.

Diferentes organismos gubernamentales y no gubernamentales y agencias del sistema de Naciones Unidas recomiendan la adopción de perfiles nutricionales ${ }^{72728}$. La evidencia señala que se han desarrollado al menos 94 perfiles nutricionales a nivel mundial, con distintos objetivos de aplicación y criterios nutricionales ${ }^{29} 30$. En Argentina aún no se ha adoptado un perfil de nutrientes, lo cual representa una barrera para la implementación de políticas alimentarias como sistemas de etiquetado frontal en los envases de alimentos. 


\section{6- Fundamentación}

Dada la alta prevalencia de sobrepeso y obesidad en Argentina es urgente la promoción de políticas que transformen el ambiente obesogénico actual en oportunidades para promover un consumo mayor de alimentos nutritivos, entre ellas la implementación obligatoria de un etiquetado frontal en los alimentos y bebidas envasados.

El objetivo del etiquetado frontal es garantizar una información veraz, simple, rápidamente visible y clara, respecto del contenido nutricional de los alimentos con el fin de promover elecciones saludables y de este modo contribuir a lograr un patrón de consumo saludable. Esta medida requiere la existencia de un perfil nutricional sólido y con robustez científica que clasifique a los alimentos de forma congruente con los mensajes de las guias alimentarias ${ }^{826}$.

Este estudio aporta evidencia local necesaria para la adopción de un perfil nutricional a nivel nacional que sirva como herramienta para el diseño e implementación de políticas de etiquetado frontal. 


\section{7- Marco teórico}

Perfiles Nutricionales y sistemas de etiquetado frontal

La necesidad de diseñar e implementar políticas que transformen el ambiente obesogénico actual en oportunidades para promover un consumo mayor de alimentos nutritivos, ha dado lugar al desarrollo de distintos perfiles nutricionales, definidos por la OMS como "la ciencia de categorizar o clasificar los alimentos según su composición nutricional por razones relacionadas con la prevención de enfermedades y la promoción de la salud ${ }^{235}$.

Durante los últimos años se han desarrollado numerosos perfiles nutricionales como herramienta para una amplia gama de política que se diferencian en los objetivos inmediatos de su aplicación, la metodología de evaluación empleada, el método de clasificación de los alimentos, y en la unidad de referencia empleada ${ }^{29} 30$.

Se usan como instrumentos en la regulación del uso de claims de nutrición y salud y de sistemas de etiquetado frontal en los envases de alimentos, en el uso de políticas fiscales para incentivar el consumo de alimentos saludables y desincentivar el consumo de alimentos no saludables, en la regulación de la publicidad de alimentos no saludables dirigida a niños, en la provisión de alimentos en instituciones para promover entornos saludables, y en campañas educativas. Cualquiera de estas medidas regulatorias requiere la existencia de criterios objetivos que categoricen a los alimentos según su calidad ${ }^{30}$.

Los perfiles nutricionales pueden ser utilizados para una función específica como es el caso del perfil nutricional creado por de la oficina regional de Europa de la OMS para regular la publicidad de alimentos no saludables dirigida a niños, o para múltiples funciones como por ejemplo el perfil nutricional desarrollado por la OPS como herramienta para el diseño e implementación de diversas estrategias reglamentarias relacionadas con la prevención y el control de la obesidad y el sobrepeso ${ }^{27} 28$.

Uno de los usos más comunes de los perfiles de nutrientes en los países de Latinoamérica ha sido el de los sistemas de etiquetado frontal en los alimentos. Esta política consiste en traducir los perfiles nutricionales de los alimentos y las bebidas en formas gráficas simples, fácil de encontrar y de entender para ayudar a los consumidores a comprender mejor la composición de nutrientes de los alimentos y, sobre la base de este entendimiento, identificar los alimentos que son opciones más saludables ${ }^{31}$. Su implementación promueve una selección informada de alimentos y desmotiva el consumo de productos con alto contenido de grasa, azúcar y sal, colaborando a reducir su impacto negativo en el estado de salud de la población ${ }^{32}$.

Los principios del CODEX, establecen que el etiquetado nutricional debe facilitar información al consumidor sobre los alimentos para que pueda elegir su alimentación con discernimiento. Establece que la expresión de datos debe ser clara, bien visible, indeleble y fácil de leer para el consumidor. La puesta en marcha del etiquetado frontal permite dar cumplimiento pleno a los principios del CODEX ${ }^{33}$. Además en el 2017 se conformó un grupo de 
trabajo electrónico para trabajar sobre un documento con orientaciones para el uso del etiquetado nutricional en el Frente del Envase ${ }^{34}$.

A nivel global, hay múltiples experiencias de carácter voluntario como por ejemplo en el Reino Unido, Australia, Nueva Zelanda, Francia, entre otros. En materia regulatoria la región de Latinoamérica es la más avanzada con leyes implementadas en México, Chile, Perú y Ecuador y varios países con iniciativas en proceso como Bolivia, Uruguay y Brasil ${ }^{35}$.

Existen distintos formatos de sistemas de etiquetado frontal que se clasifican según el grado de información que se brinda al consumidor y el nivel de interpretación requerido para identificar cuán saludable es un producto.

Algunos autores los clasifican en sistemas de resumen y en sistemas basados en nutrientes específicos ${ }^{36}$. Los sistemas de resumen combinan los niveles de nutrientes para dar una calificación general del valor nutricional de un producto. La mayoría de consiste en logos que indican cuando un producto es "saludable" en base a un perfil de nutrientes. Algunos ejemplos son el sistema nórdico (Keyhole), el logo choices international, entre otros ${ }^{37}$. Otros posicionan al producto en una escala de color que representa la calidad nutricional como el sistema Nutriscore (o 5-Colour Nutrition Label) ${ }^{38}$.

Los sistemas basados en nutrientes específicos muestran información específica para un número limitado de nutrientes críticos. Por ejemplo, Chile introdujo recientemente una etiqueta de advertencia obligatoria en alimentos con alto contenido de calorías, azúcares, sodio o grasas saturadas $^{39}$. El Reino Unido tiene un sistema voluntario de "semáforo" que utiliza colores (rojo, amarillo y verde) para clasificar el contenido de grasas totales, grasas saturadas, azúcares y sal (sodio) como contenido alto, medio o bajo en un alimento ${ }^{40}$.

Otros autores clasifican a los sistemas de etiquetado frontal en sistemas directivos, semidirectivos y no directivos ${ }^{41}$. Los sistemas no directivos, como el sistema de ingesta diaria recomendada (o GDA por sus siglas en inglés), únicamente contienen información sobre el contenido de nutrientes por porción de alimento, no permitiéndole a la población mejorar su comprensión de la información nutricional. Los sistemas semi-directivos como el semáforo, le indican al consumidor el contenido de nutrientes utilizando un código de color y texto explicativo. Estos sistemas no realizan un juicio de valor sobre cuán saludables son los alimentos y requieren que los consumidores elaboren un juicio global a partir de los niveles de cada uno de los nutrientes presentados. Finalmente, los sistemas directivos, tales como el sistema de advertencias, identifican productos poco saludables, sin presentar mayor detalle sobre el contenido de nutrientes ${ }^{42}$.

La evidencia muestra que los sistemas más efectivos para la identificación de los productos más saludables por parte de los consumidores son los sistemas basados en nutrientes específicos respecto a los sistemas de resumen ${ }^{36}$, y los sistemas semi-directivos y directivos respecto a los no directivos ${ }^{42} 434445$. 
Guías Alimentarias

En la Declaración Mundial sobre Nutrición y el Plan de Acción para la Nutrición aprobada en la Conferencia Internacional sobre Nutrición de $1992^{46}$ se determinó como prioritario el desarrollo y la implementación de Guías Alimentarias Basadas en Alimentos (GABA) cuyas directrices fueron publicadas en 1996 por $\mathrm{FAO} / \mathrm{OMS}^{47}$.

La OMS define a las GABA como directrices basadas en evidencia científica para ayudar a las personas a lograr un patrón de alimentación saludable. Adquieren un rol trascendental como herramienta para lograr las recomendaciones nutricionales traduciendo las metas nutricionales en alimentos mediante un patrón de consumo saludable 5 . Ya han sido desarrolladas en más de 100 países para asesorar al público en general sobre alimentos, grupos de alimentos y patrón de consumo para promover la salud general y prevenir la obesidad y las ECNT ${ }^{48}$.

En Argentina las GAPA son de carácter nacional, se dirigen a la población general mayor a 2 años y tienen como objetivo traducir las metas nutricionales establecidas para la población en mensajes prácticos que contemplen factores epidemiológicos, sociales, económicos y culturales que alientan a mejorar el perfil de hábitos y consumo de alimentos de la población. La primer versión se elabora en el 2000 y fueron actualizadas en el 2015 luego de un proceso de revisión exhaustivo con participación de actores intersectoriales. Los cambios realizados en la última actualización se basan en la situación alimentario - nutricional y epidemiológica de todo el país. que muestra un aumento en la prevalencia de sobrepeso y obesidad y otras ECNT relacionadas a un patrón de consumo conformado de forma predominante con alimentos con alto contenido en nutrientes críticos como azúcar, sodio y grasas.

No se dirigen sólo al ámbito individual, sino también al ámbito gubernamental y al de la industria de alimentos. Son un insumo fundamental para la formulación de políticas relacionadas con la alimentación y la nutrición. Las políticas de salud y agroalimentarias deberían hacer sinergia para que la población pueda lograr el patrón alimentario recomendado por las GAPA. Además deberían orientar a la industria en la formulación de la calidad nutricional de los alimentos y en el diseño del etiquetado nutricional.

Las GAPA traducen los mensajes sobre una alimentación saludable mediante 10 mensajes y una gráfica de alimentación saludable que la complementan. Los mensajes son: 1) Incorporar a diario alimentos de todos los grupos y realizar al menos 30 minutos de actividad física, 2) Tomar a diario 8 vasos de agua segura, 3) Consumir a diario 5 porciones de frutas y verduras en variedad de tipos y colores, 4) Reducir el uso de sal y el consumo de alimentos con alto contenido de sodio, 5) Limitar el consumo de bebidas azucaradas y de alimentos con elevado contenido de grasas, azúcar y sal, 6) Consumir diariamente leche, yogur o queso, preferentemente descremados, 7) Al consumir carnes quitarle la grasa visible, aumentar el consumo de pescado e incluir huevo, 8) Consumir legumbres, cereales preferentemente integrales, papa, batata, choclo o mandioca, 9) Consumir aceite crudo como condimento, frutas 
secas o semillas, 10) El consumo de bebidas alcohólicas debe ser responsable. Los niños, adolescentes y mujeres embarazadas no deben consumirlas.

La "Gráfica de la Alimentación Diaria" incluye a los alimentos agrupados de acuerdo a los nutrientes que los componen principalmente y representa la síntesis de la frecuencia diaria de consumo recomendada para cada grupo de alimento.: Grupo: 1 Frutas y Verduras; Grupo: 2 Legumbres, cereales, papa, pan y pastas; Grupo: 3 leche, yogur y queso; Grupo: 4 carnes y huevos, Grupo 5: aceite, frutas secas y semillas; Grupo 6: Alimentos de consumo opcional. Para el diseño de la gráfica se tuvo en cuenta para cada grupo el espacio proporcional que representa las porciones diarias recomendadas. Dentro del mismo grupo, los alimentos son intercambiables entre sí, contribuyendo a la variedad.

El patrón alimentario recomendado por las GAPA clasifica a los alimentos en dos grandes grupos denominados "alimentos protectores" y "alimentos de consumo opcional" según su calidad nutricional y su frecuencia de consumo recomendada. Los alimentos protectores incluyen los grupos del 1 al 5 y son aquellos alimentos que aportan nutrientes esenciales y que consumidos de manera adecuada y proporcionada ayudan a vivir con mejor salud. Los alimentos de consumo opcional incluidos en el grupo 6 son aquellos que se deben consumir en pequeñas cantidades y en menor frecuencia de consumo, por su alta cantidad de calorías y por su baja calidad nutricional. Su aporte calórico debería representar un máximo de $15 \%$ de las calorías totales. El consumo en exceso de estos alimentos predispone a la obesidad, hipertensión, diabetes, enfermedades cardiovasculares y aumenta el riesgo de desarrollar caries, cáncer. Incluye bebidas azucaradas, galletitas, snacks, golosinas, cereales de desayuno azucarados, entre otros.

Además las GAPA realizan recomendaciones en base al nivel de procesamiento de los alimentos según el sistema NOVA ${ }^{49}$, alentando el consumo de las comidas caseras y saludables a base de alimentos naturales o mínimamente procesados por sobre los alimentos procesados y ultra procesados.

\section{$\underline{\text { Relación de los perfiles nutricionales con las Guías Alimentarias }}$}

La OMS conformó un grupo de expertos para proporcionar orientación en el desarrollo o adaptación de modelos de perfil de nutrientes. Recomienda validar los perfiles nutricionales que existen hasta ahora para evaluar que sean sean efectivos y adecuados para el contexto en el que se van a utilizar. El objetivo que los perfiles sean coherentes con y consistentes con los mensajes de nutrición de salud pública para lograr, en última instancia, mejorar la nutrición y la salud pública ${ }^{50} 8$.

Se sugieren distintos enfoques para para validar un sistema de perfiles de nutrientes: el análisis de la clasificación de los alimentos frente a un punto de referencia que puede ser un acuerdo de expertos o las GABA; la evaluación de la capacidad del perfil de nutrientes para 
medir adecuadamente la calidad de la dieta de los individuos, y la asociación prospectiva del índice dietético individual con el estado nutricional y los resultados de salud ${ }^{8}$

La primer fase corresponde a evaluar la clasificación de los alimentos con relación a las recomendaciones nutricionales. Al igual que las guías alimentarias, los perfiles nutricionales deben contribuir a través de su aplicación a cumplir las ingestas de nutrientes recomendadas y se deben adaptar al patrón alimentario de cada país y a los problemas particulares de salud pública relacionados. Las guías alimentarias son recomendaciones para una alimentación saludable enmarcada en términos de alimentos y un patrón de alimentación. Los perfiles nutricionales clasifican los alimentos en función de su composición nutricional y a través de su uso en políticas alimentarias contribuyen potencialmente a lograr las recomendaciones nutricionales. Por lo tanto, es constructivo que los perfiles nutricionales se correspondan con los mensajes y con el modelo de patrón alimentario propuestos por las guías alimentarias en las regiones o países en los que se aplican ${ }^{8}$. Es decir que los alimentos promovidos por las guías, también deberían resultar promovidos por el perfil de nutrientes y los considerados como alimentos "a limitar" o a moderar su consumo según los perfiles deberían corresponderse con aquellos alimentos que las guías recomiendan consumir con frecuencia ocasional o moderada.

La coherencia las políticas es importante para proporcionar mensajes alimentarios consistentes y lograr de esta manera un mayor impacto en la salud pública, promoviendo la credibilidad y la sostenibilidad de ambas políticas ${ }^{51}$.

La literatura con respecto a la validación de perfiles nutricionales es limitada. Hasta la fecha el perfil más evaluado para su aplicación en otros países es el perfil de nutrientes del Reino Unido desarrollado en 2005 por un equipo de investigación de Oxford con el objetivo de regular la publicidad destinada a los niños y validado por la Agencia de Normas Alimentarias del Reino Unido $^{52}{ }^{53}$. Actualmente se utiliza en la regulación de la publicidad de alimentos en Irlanda, uso de claims nutricionales en Australia, Nueva Zelanda ${ }^{54}$ y Sudáfrica $^{55}$; y en políticas de etiquetado frontal en Australia ${ }^{51}$, Nueva Zelanda ${ }^{56}$ y Francia $^{57}$.

Previo a su aplicación, los países evaluaron su pertinencia en la clasificación de los alimentos con relación a sus GABA. En el Reino Unido y en Sudáfrica el perfil presenta un acuerdo sustancial con sus respectivas GABAs con un coeficiente kappa 0,69 y 0,73 respectivamente ${ }^{558}$. En Francia detectaron que dicho perfil permite clasificar los grupos alimentarios de manera congruente con las recomendaciones nutricionales de salud pública: las frutas y verduras y los productos a base de cereales se clasifican en las primeras clases de la puntuación (mejor calidad nutricional), mientras que los snacks dulces y salados se clasifican más bien en las últimas clases (menor calidad nutricional). A partir de los resultados se realizaron adaptaciones en el perfil para algunas categorías de alimentos como los frutos secos, bebidas, materias grasas y quesos a fin de garantizar una mejor concordancia con las recomendaciones nutricionales de salud pública ${ }^{596061}$. 
En Nueva Zelanda y Australia se ha realizado un proceso similar al de Francia, se evaluó el perfil de nutrientes sobre más de 10.000 alimentos locales y se realizaron las modificaciones necesarias para lograr que el perfil del Reino Unido clasifique satisfactoriamente los alimentos de todas las categorías de manera acorde a las recomendaciones de las guías alimentarias para ser utilizado como heraamienta para ${ }^{62} 63$. El sistema de etiquetado frontal denominado "Health Star Rating System" se implementó en ambos países en Junio 2014 de manera voluntaria.

Luego de cuatro años de implementación de la politica de etiquetado frontal en Australia, un estudio revisó la consistencia del perfil con sus GABA. Se evaluó la calidad nutricional de una muestra de 65.660 alimentos envasados según sus GABA y según el perfil de nutrientes, clasificando a los alimentos en alimentos "a promover" y "a limitar". Se observó una porcentaje de acuerdo del $86.6 \%$. De los productos que presentaron desacuerdo, se determinó un 83\% como fallas de las GABA en la clasificación de los productos, y un 7.0\% como fallas del perfil de nutrientes. Se establecieron recomendaciones específicas para fortalecer ambas políticas ${ }^{51}$.

En Candá validaron el perfil nutricional de los claims nutricionales para ser utilizado en un sistema de etiquetado frontal. El perfil utiliza como punto de corte el 15\% del valor diario (VD) de la ingesta diaria recomendada de nutrientes para indicar si un alimento tiene "alto contenido" en un nutriente a promover como el calcio. Para validar si el mismo punto de corte sirve para una política de etiquetado frontal que indique si un alimento tiene "alto contenido" en nutrientes "a limitar" como sodio, azúcar y grasa, se aplicó el perfil con distintos puntos de corte (10 al $25 \%$ del VD) en una base de datos de composición de alimentos de aproximadamente 350 alimentos indicadores, comprobando que el umbral del 15\% es el de mayor coherencia con el patrón de alimentación saludable recomendado por las GABAs de Canadá. Los alimentos que deberían consumirse con mayor frecuencia (como frutas y verduras), no tendrían que mostrar un advertencia, mientras que la mayoria de los alimentos que se recomienda limitar su consumo (como gaseosas, galletitas, helados y salchichas), probablemente mostrarían uno o más advertencias ${ }^{4}$. Además, se realizó un análisis de dietas simuladas conformadas por alimentos frecuentemente consumidos y compatibles con la GABA de Canadá, que demostró que elegir alimentos por debajo del $15 \%$ del VD por porción ayudaría a los canadienses a mantener su ingesta de nutrientes críticos, como el sodio, por debajo de los niveles que contribuyen a las enfermedades crónicas relacionadas con la dieta ${ }^{65}$. 


\section{8- Objetivo}

Evaluar el nivel de concordancia de los perfiles nutricionales de la región de Latinoamérica con las Guías Alimentarias para la Población Argentina. 


\section{9- Diseño y metodología}

Diseño

Estudio observacional analítico de corte transversal. El universo incluyó los perfiles nutricionales desarrollados en la región de Latinoamérica.

Unidad de análisis

Cada uno de los perfiles nutricionales que cumplieron con los siguientes criterios de inclusión:

- Desarrollados para la región de Latinoamérica;

- Desarrollados con el objetivo de servir de herramienta en el diseño e implementación de políticas de sistemas de etiquetado frontal semi-directivos y directivos de carácter obligatorio;

- Que se encuentren definidos en normativas sancionadas y/o implementadas o en proyectos de decreto que se encuentren en consulta pública.

El estudio incluye dos actividades principales:

1) Mapeo de perfiles nutricionales de la región de Latinoamérica durante el mes de mayo y noviembre del año 2017.

2) Desarrollo del listado de alimentos locales con su respectiva información nutricional y evaluación de la calidad nutricional con los perfiles nutricionales y las GAPA.

A continuación se detallan los aspectos metodológicos de cada actividad desarrollada.

\section{1) Mapeo de perfiles nutricionales de la región de Latinoamérica}

\section{Recolección de datos}

Los perfiles nutricionales desarrollados en la región de Latinoamérica con el objetivo de servir de herramienta en el diseño e implementación de políticas de etiquetado frontal.

Con el propósito de identificar los perfiles se relevaron las siguientes fuentes secundarias: leyes, reglamentaciones, proyectos de ley existentes en la región, manuales de Organizaciones Internacionales de Salud Pública (OMS y OPS) con información acerca de perfiles nutricionales diseñados en la región de América Latina. Además se seleccionaron artículos científicos que describieron a los perfiles identificados.

La búsqueda se realizó a través de páginas oficiales de gobierno, y a través de motores de búsqueda como Google, Google Scholar y PubMed.

La búsqueda de información se realizó entre el mes de mayo y noviembre del año 2017.

\section{Definición operacional de las variables}

Para cada perfil se analizaron las siguientes variables seleccionadas en base al modelo "Funnel" desarrollado para tal fin ${ }^{66}$. 
a. País/Región: Los países/regiones en donde se utiliza y/o se utilizará el perfil. Variable abierta.

b. Organismo/Entidad: nombre del organismo/entidad que desarrolla el perfil (OPS, OMS, ONGs, instituciones académicas, etc.). Variable abierta.

c. Intervención de política pública: Los perfiles se utilizan como herramienta en políticas públicas. Según la política en la que se usen se pueden clasificar en: Producto (reformulación), Plaza (oferta en escuelas), Promoción (etiquetado frontal, uso de claims), Precio (políticas de subsidios/impuestos). Algunos perfiles tienen más de un propósito. Variable categórica: Producto/Plaza/ Promoción/Precio.

d. Normativa: Regulación donde se definen los perfiles y el sistema de etiquetado frontal. Variable abierta.

e. Estado normativo: pueden estar sancionadas y/o implementadas o en proyectos de decreto que se encuentren en consulta pública. Variable abierta.

f. Carácter/Uso: los perfiles se aplican sobre la base de una utilización voluntaria u obligatoria. Variable categórica: voluntario/obligatorio.

g. Clasificación según directividad: Existen varios formatos de sistemas de etiquetado frontal los cuales difieren en el grado en que instruyen al consumidor sobre cuán saludable es un producto. Los sistemas no directivos, como el sistema de ingesta diaria recomendada (o GDA por sus siglas en inglés), únicamente contienen información sobre el contenido de nutrientes por porción de alimento, no permitiéndole a la población mejorar su comprensión de la información nutricional. Los sistemas semi-directivos como el semáforo, le indican al consumidor el contenido de nutrientes utilizando un código de color y texto explicativo. Estos sistemas no realizan un juicio de valor sobre cuán saludables son los alimentos y requieren que los consumidores elaboren un juicio global a partir de los niveles de cada uno de los nutrientes presentados. Finalmente, los sistemas directivos, tales como el sistema de advertencias, identifican productos poco saludables, sin presentar mayor detalle sobre el contenido de nutrientes ${ }^{41}$. Variable categórica: directivo/semi-directivo/no directivo

h. Símbolo: Cada sistema de etiquetado frontal está representado por un símbolo específico o un logotipo (ejemplo: semáforo, advertencias,). Variable categórica: semáforo/advertencia.

i. Tipo de alimentos a los que se aplica el perfil: Los perfiles se pueden aplicar en alimentos según su grado de procesamiento/agregado de ingredientes. La mayoría se aplican a los alimentos procesados con agregado de los nutrientes críticos en el procesamiento, excluyendo a los alimentos naturales o mínimamente procesados. Variable categórica: todos los alimentos/solo procesados.

j. Enfoque por categorías o transversal: El enfoque por categorias es cuando el perfil tiene una aplicación que varía según la categoría de alimentos (distintos límites y/o distintos nutrientes) y el enfoque transversal se aplica por igual a todos los alimentos.Variable categórica: categoria/transversal. 
k. Enfoque por umbrales (threshold model) o sistema de puntuación (scoring model): los perfiles pueden utilizar dos métodos de medición, puntuación o umbrales. Los sistemas de puntuación asignan una puntuación global al alimento obtenido al combinar el puntaje de nutrientes/ingredientes "a limitar" y el puntaje de nutrientes/ingredientes a promover. En el método de medición de umbral, define para cada nutriente/ingrediente un umbral. Variable categórica: umbral/puntuación.

1. Cantidad de resultados: El resultado del perfil puede ser doble (alto/cantidad excesivas o bajo/no contiene cantidad excesiva) o triple (alto/medio/bajo). Variable categórica: doble/triple.

$\mathrm{m}$. Enfoque de riesgo o beneficio: un i puede incluir ingredientes/nutrientes a promover (enfoque de beneficio) y/o "a limitar" (enfoque de riesgo). Los ingredientes/nutrientes a promover contribuyen positivamente a la salud y los ingredientes/nutrientes "a limitar" tiene un impacto negativo en la salud. Algunos perfiles usan exclusivamente ingredientes/nutrientes a promover o "a limitar", mientras que otros sistemas usan una combinación de ambos. Variable categórica: riesgo/beneficio/ambos.

n. Nutrientes/ingredientes: Los perfiles pueden incluir diferentes nutrientes $y / 0$ ingredientes como azúcares, sodio, grasas saturadas, grasas trans, grasas totales, calorías, etc. Variable abierta.

o. Unidad de referencia: Algunos perfiles utilizan una unidad estándar como $100 \mathrm{~g}$-ml, porción o total de calorías del alimento. Variable categórica: 100 g/porción/total de calorías del alimento.

p. Líquido/sólido: Los perfiles pueden establecer límites distintos según el producto se consuma sólido o líquido. Variable dicotómica: sí/no. (si: límites distintos para líquido y para sólido, no: límites iguales para líquido y para sólido)

q. Gradualidad: Algunos perfiles se aplican con diferentes niveles de gradualidad en un periodo de tiempo determinado, progresando los límites hacia valores más exigentes. Variable dicotómica: sí/no. (si: límites con gradualidad, no: límites sin gradualidad)

Algunas normativas establecen propuestas de gradualidad en sus puntos de corte. En este estudio se tomará el 1er nivel de gradualidad propuesto o el que se esté implementando actualmente.

2) Desarrollo del listado de alimentos locales con su respectiva información nutricional y evaluación de la calidad nutricional con los perfiles nutricionales y las GAPA.

Población accesible. Muestra. Selección y tamaño de la muestra

Se unificó la base de datos de todos los productos con información nutricional comprendidos en las bases de composición de alimentos de FIC Argentina ( $\mathrm{n}=2001)$. En el caso de aquellas categorías de alimentos que no estaban incluidas en la base de datos de FIC Argentina (Legumbres, Hortalizas feculentas, Frutas, Carnes, Huevo, Leche entera, Leche parcialmente descremada, Aceite, Frutos secos, Semillas, Azúcares libres, Soda, Aguas 
Minerales, Infusiones) se tomó como referencia los productos y la información nutricional disponible en las Tablas de Composición Nutricional Argenfoods $(n=18)^{67}$.

Se clasificaron los productos según los grupos de alimentos establecidos en las GAPA: 1)legumbres, cereales, papa, pan y pastas; 2)frutas y verduras; 3)carnes y huevo; 4)leche, yogur y quesos; 5)aceite crudo, frutos secos y semillas; 6)alimentos de consumo opcional. Además se agregaron 2 grupos para los productos que no se incluían en los grupos mencionados en las GAPA: 7) bebidas sin azúcar y 8) comidas pre-elaboradas (Combinación de alimentos pertenecientes a distintos grupos) (Tabla 1).

Tabla 1: Grupos y categorías de alimentos GAPA. 2016.

\begin{tabular}{|c|c|c|}
\hline Grupo de alimento & Categorías de alimentos & Ejemplos de alimentos incluidos \\
\hline \multirow{10}{*}{$\begin{array}{c}\text { Legumbres, } \\
\text { cereales, papa, pan } \\
\text { y pastas }\end{array}$} & Legumbres & lentejas, garbanzos, porotos, soja, arvejas \\
\hline & Legumbres enlatadas & \\
\hline & $\begin{array}{l}\text { Cereales refinados: arroz, } \\
\text { fideos, pastas }\end{array}$ & arroz, fideos, pastas \\
\hline & Cereales integrales & Arroz integral, avena, germen de trigo \\
\hline & Pan blanco & $\begin{array}{l}\text { Lactal sin salvado, pan de pancho, pan de hamburguesa, } \\
\text { pan francés }\end{array}$ \\
\hline & Pan integral & Lactal con salvado, semillas y cereales \\
\hline & Hortalizas feculentas & Papa, batata, choclo y mandioca \\
\hline & Pastas rellenas & Ravioles, sorrentinos, capelettis \\
\hline & $\begin{array}{l}\text { Premezclas panificados y } \\
\text { cereales }\end{array}$ & pan, puré de papa, masa de pizza, ñoquis \\
\hline & Amasados & $\begin{array}{l}\text { Refrigerados y congelados: panqueques, tapas de tartas, } \\
\text { tapas de empanadas, pan, masa de pizza }\end{array}$ \\
\hline \multirow[t]{5}{*}{ Frutas y verduras } & Frutas & $\begin{array}{l}\text { Frutas como único ingrediente sin aditivos, frescas o } \\
\text { congeladas. }\end{array}$ \\
\hline & Verduras & $\begin{array}{l}\text { Verduras como único ingrediente sin aditivos, frescas o } \\
\text { congeladas. }\end{array}$ \\
\hline & Verduras y frutas enlatadas & \\
\hline & Verduras en salmuera & aceitunas, pepinos \\
\hline & Comidas listas de verdura & Medallones verdura, rellenos de verdura \\
\hline \multirow[t]{4}{*}{ Carnes y huevo } & Carnes & carne de cerdo, ave, cordero, pescados, mariscos, etc \\
\hline & Huevo & \\
\hline & Carnes enlatadas & pescados y mariscos \\
\hline & Carnes procesadas & $\begin{array}{l}\text { Nuggets de pollo, hamburguesas, enlatados, salmueras, } \\
\text { chacinados, salchichas, bastoncitos de pescado, } \\
\text { fiambres, carnes y pescados salados, encurtidos, } \\
\text { ahumados o curados, conservas de pescado en aceite. }\end{array}$ \\
\hline $\begin{array}{l}\text { Leche, yogur y } \\
\text { quesos }\end{array}$ & Leche entera & \\
\hline
\end{tabular}




\begin{tabular}{|c|c|c|}
\hline & $\begin{array}{l}\text { Leche parcialmente } \\
\text { descremada }\end{array}$ & \\
\hline & Yogur entero & \\
\hline & $\begin{array}{l}\text { Yogur parcialmente } \\
\text { descremado }\end{array}$ & \\
\hline & $\begin{array}{l}\text { Queso duro, semiduro y } \\
\text { saborizados }\end{array}$ & \\
\hline & $\begin{array}{l}\text { Quesos blandos (no } \\
\text { saborizados) }\end{array}$ & \\
\hline \multirow{3}{*}{$\begin{array}{l}\text { Aceite crudo, frutos } \\
\text { secos y semillas }\end{array}$} & Aceite & Oliva, girasol, maíz \\
\hline & Frutos secos & Nuez, almendras, maní, otros \\
\hline & Semillas & Girasol, calabaza, lino, chía, otros \\
\hline \multirow{15}{*}{$\begin{array}{c}\text { Alimentos de } \\
\text { consumo opcional }\end{array}$} & Alimentos grasos & Mayonesa, salsa golf, crema, manteca \\
\hline & Azúcares libres & Azúcar, dulces, mermeladas \\
\hline & Alimentos dulces & Golosinas, chocolates, helados, alfajores \\
\hline & Galletitas & Dulces, saladas, de agua \\
\hline & Postres azucarados & a base de leche, deshidratados, gelatinas \\
\hline & Bebidas azucaradas & Gaseosas, aguas saborizadas, jugos, etc. \\
\hline & Panificados dulces o salados & Budines, tortas \\
\hline & $\begin{array}{l}\text { Premezclas panificados } \\
\text { dulces }\end{array}$ & torta fritas, biscochuelos \\
\hline & Barras de cereal con azúcar & Frutal, con chocolate, otros \\
\hline & $\begin{array}{l}\text { Cereales de desayuno con } \\
\text { azúcar }\end{array}$ & Copos de maíz, con fibra, infantiles, otros \\
\hline & $\begin{array}{llll}\text { Snacks } & \text { y productos } & \text { de } \\
\text { copetín } & & & \end{array}$ & $\begin{array}{l}\text { Papas fritas, palitos, galletitas snack, frutos secos con } \\
\text { sal/azúcar }\end{array}$ \\
\hline & Comidas rápidas & $\begin{array}{l}\text { Platos de pasta, hamburguesas, pizza, tortilla papa, } \\
\text { empanadas, tacos, papas fritas congeladas (todos } \\
\text { envasados, no caseros), milanesas de soja, hamburguesas } \\
\text { de soja }\end{array}$ \\
\hline & Aderezos & Ketchup, mostaza, pasta de aceituna, salsa de soja, otros \\
\hline & Salsas comerciales & enlatadas, en sachet o deshidratadas \\
\hline & $\begin{array}{l}\text { Caldos, saborizadores y } \\
\text { sopas comerciales }\end{array}$ & \\
\hline \multirow[t]{4}{*}{ Bebidas sin azúcar } & Soda & \\
\hline & Aguas minerales & \\
\hline & Infusiones & te, mate, café \\
\hline & Bebidas edulcoradas & Gaseosas, aguas saborizadas, jugos, etc. \\
\hline $\begin{array}{l}\text { Comidas pre- } \\
\text { elaboradas }\end{array}$ & & $\begin{array}{l}\text { tartas, carnes con verduras, sándwiches (todos envasados, } \\
\text { no caseros) }\end{array}$ \\
\hline
\end{tabular}

Fuente: Elaboración propia en base a las GAPA 2016. 
Se realizó una limpieza de la base de datos en donde se eliminaron los productos sin el dato de unidad de porción, los productos que no pertenecían a los grupos y categorías de alimentos de las GAPA ( $\mathrm{n}=134)$, y los productos duplicados en las distintas recolecciones (misma marca, nombre e información nutricional) $(\mathrm{n}=182)$. En el caso de productos con misma marca y nombre pero distinta información nutricional, se dejó el producto con fecha de recolección más actual.

Figura 1: Flujograma de conformación de la muestra total.

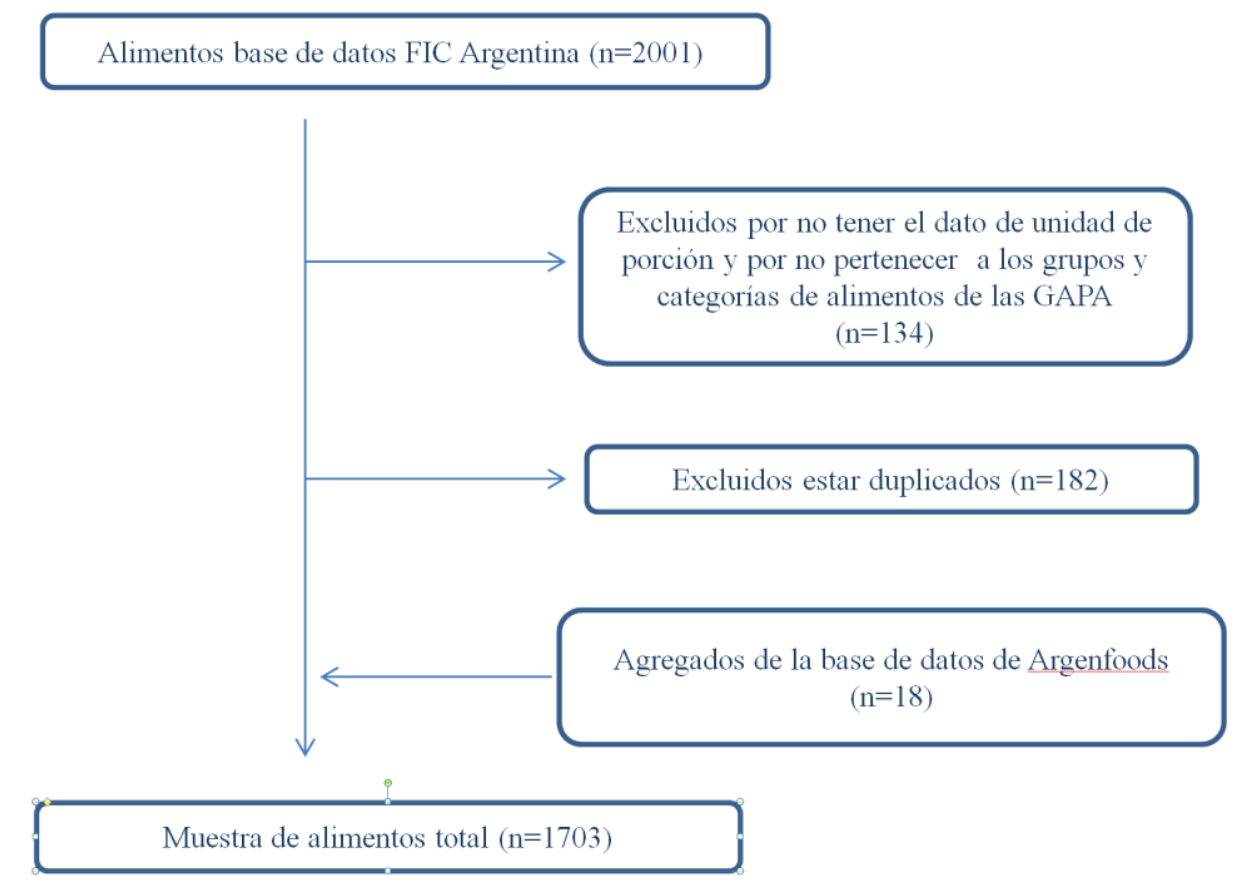

Fuente: Elaboración Propia.

\section{Definición operacional de las variables y categorías}

Calidad nutricional del producto: se ha evaluado a partir de la clasificación de las GAPA y la clasificación de los perfiles nutricionales.

- Clasificación GAPA: las GAPA traducen las metas alimentario-nutricionales para la población en mensajes prácticos que permiten clasificar a los alimentos en "alimentos a promover", "de consumo moderado" o "a limitar" en base a la situación alimentario- nutricional y epidemiológica de todo el país 5 .

Para realizar dicha clasificación se ha desarrollado un protocolo que ha sido revisado y validado por licenciados en nutrición expertos en salud pública (Lic. Andrea Graciano, Lic. Ignacio Agustin Mendez Gonzalez y Magister Celeste Nessier) (Ver ANEXO I).

Variable categórica: Alimentos a promover: 1; Alimentos de consumo moderado:2; Alimentos a limitar: 3

Alimentos a promover: Alimentos que conforman el grupo de alimentos protectores, y dentro del grupo al cual pertenecen se recomienda preferentemente su consumo debido a su 
calidad nutricional superior. Ejemplo: cereales integrales, leche descremada, frutas y verduras frescas, agua, etc.

Alimentos de consumo moderado: Alimentos que conforman el grupo de alimentos protectores, y dentro del grupo al cual pertenecen no se prioriza su consumo debido a su calidad nutricional inferior. Ejemplo: cereales refinados, leche entera, frutas y verduras enlatadas, bebidas edulcoradas, etc.

Alimentos a limitar: Alimentos que se recomienda reducir su consumo y aquellos que conforman el grupo de alimentos de consumo opcional. Ejemplo: galletitas, postres azucarados, verduras en salmuera, bebidas azucaradas, etc.

- Clasificación según perfiles nutricionales: Los Perfiles Nutricionales son herramientas que permiten clasificar a los alimentos según su calidad nutricional en alimentos ${ }^{10}$. Se utilizaron perfiles que sirven de herramienta en sistemas de etiquetado frontal de advertencia y en sistemas de semáforo.

Los perfiles de los sistemas de etiquetado frontal de advertencia clasifican a los alimentos en:

Alimentos a promover: cuando todos los nutrientes críticos se encuentran por debajo del límite alto establecido por el perfil.

Alimentos a limitar: Cuando al menos un nutriente crítico supera el límite alto establecido por el perfil.

Sin dato: Cuando al menos un nutriente crítico no presenta el dato excepto para azúcares totales y libres*.

Los perfiles de los sistemas de etiquetado frontal de semáforo clasifican a los alimentos en:

Alimentos a promover: cuando todos los nutrientes críticos se encuentran por debajo de los límites (alto y medio) establecidos por el perfil.

Alimentos de consumo moderado: cuando todos los nutrientes críticos se encuentran por debajo de los límites "alto" y al menos un nutriente supera el límite medio establecidos por el perfil.

Alimentos a limitar: Cuando al menos un nutriente crítico supera el límite alto establecido por el perfil.

Sin dato*: Cuando al menos un nutriente crítico no presenta el dato excepto para azúcares totales y libres.

\section{$\underline{\text { Recolección de datos }}$}

\footnotetext{
* Azucares totales y libres sin dato: Solo se considera sin dato si el producto pertenece a una categoría de alimentos donde los productos suelen tener alto contenido de azúcares como yogur entero, mermeladas y dulces, alimentos dulces, galletitas, postres azucarados, bebidas azucaradas, panificados dulces o salados, premezclas panificados dulces, barras de cereal con azúcar, cereales de desayuno con azúcar y aderezos y no se declara el azúcar.
} 
La base de datos de FIC Argentina se desarrolló como parte de tres investigaciones: a) "Estudio para conocer el contenido de sodio y grasas trans de los alimentos procesados de Argentina"68, como parte de una iniciativa colaborativa internacional liderada por The George Institute $^{69}$, Australia para monitorear el contenido nutricional de los alimentos procesados en todo el mundo. b) "Estudio sobre la publicidad de alimentos no saludables dirigida a niños en la TV argentina"70; c) "Estudio de Rotulado facultativo y técnicas de marketing dirigidas a niños en envases de alimentos procesados de Argentina" ${ }^{, 11}$.

La recolección de datos para conformar la base se realizó entre el 2014 y el 2016 en cadenas de supermercados de Buenos Aires seleccionadas por ser las cadenas líderes que operan en el país. Para la recolección de datos se tomaron fotografías de todos los productos expuestos en las góndolas o exhibidores del supermercado visitado (técnica de barrido) incluidos dentro de las categorías seleccionadas. Se incluyeron todas las marcas y presentaciones disponibles de los productos dentro de los grupos de alimentos seleccionados: cereales y derivados; panes y productos de panadería; productos cárnicos y derivados; yogures y quesos; postres a base de leche; chocolates; comidas rápidas; conservas de frutas y verduras; snacks y aperitivos; emulsiones de aceites, salsas y aderezos; bebidas no alcohólicas; bebidas energizantes; y condimentos.

Toda la información recolectada fue ingresada en la base de datos. La composición nutricional de los alimentos evaluados fue obtenida a través de la información nutricional presente en el rotulado de los alimentos envasados.

En Argentina la declaración de azúcares totales es opcional y no se declaran los azúcares libres, por lo tanto la mayoría de los productos de la muestra no tiene el dato de este nutriente. En el caso de los azúcares totales, en las categorías de bebidas azucaradas y de alimentos dulces (golosinas) se consideró el contenido de carbohidratos declarado en el rótulo nutricional como equivalente al de azúcares totales.

El contenido de azúcares libres se calculó en toda la muestra a partir del dato de azúcares totales declarado según metodología propuesta por $\mathrm{OPS}^{27}$, que considera el siguiente porcentaje de azúcares libres a partir del total de azúcares totales declarado en el rótulo nutricional:

- 100\% del azúcar total corresponde a azúcares libres en alimentos dulces (bebidas azucaradas, cereales de desayuno, alfajores, chocolates, galletitas dulces y saladas)

- $100 \%$ del azúcar total corresponde a azúcares libres en productos que naturalmente no contienen azúcar: aderezos (kétchup y mayonesa), comidas rápidas (milanesas de soja y verdura, hamburguesa de soja, pastas, pizzas y arroces listos), salsa lista, carnes procesadas.

- $75 \%$ en barras de cereal con frutas.

- $75 \%$ en postres azucarados con leche en sus ingredientes.

- $75 \%$ panificados dulces: tapas de empanadas, panes lactales, pastas rellenas, puré de papa. 


\section{0- Plan de análisis de los resultados}

Se realizó un análisis descriptivo de los datos presentándose en tablas simples y de doble entrada. Para el procesamiento de datos y el análisis estadístico se utilizó el SPSS statistics 20.0 y el software Stata. Las principales variables que se analizaron para cada uno de los perfiles fueron: nivel de exigencia y nivel de acuerdo, utilizadas en numerosos estudios similares para evaluar perfiles nutricionales ${ }^{72} 73747576$.

\section{Nivel de exigencia}

El nivel de exigencia se mide por el porcentaje de alimentos "a limitar". Se incluyeron los alimentos "Sin dato" de la muestra para realizar dicha comparación.

Esta variable permite estimar el nivel de exigencia de los perfiles y las GAPA y determinar cuáles son más estrictos, es decir que clasifican a una mayor proporción de alimentos como "a limitar".

Se comparó la frecuencia porcentual de la clasificación de la calidad nutricional de los alimentos según GAPA y según los perfiles en el total de la muestra, por grupo y categoría de alimentos.

Además se analizó el nivel de exigencia de los perfiles por umbral de nutriente crítico en el total de la muestra y por categoría de alimentos. Esta variable estima cuáles perfiles son más estrictos por umbral de nutriente crítico, es decir clasifican a una mayor proporción de alimentos como "Alto en" determinado nutriente crítico. Se excluye a las GAPA de este último análisis porque, a diferencia de los perfiles, no establecen umbrales específicos para cada nutriente.

\section{Nivel de acuerdo}

Para medir la variación o acuerdo entre la clasificación de la calidad nutricional de los alimentos según GAPA y según perfiles nutricionales, se ha utilizado como primera aproximación el índice de concordancia expresado a través del porcentaje de acuerdo entre clasificaciones, el cual se calcula contabilizando las cantidad de coincidencias entre el perfil y las GAPA sobre el total de alimentos evaluados. Para evaluar con más precisión los niveles de acuerdo, se ha acompañado el porcentaje de concordancia con el Índice kappa de Cohen ${ }^{77}$ y el Gwet's $\mathrm{AC} 1^{78}$. El Índice Kappa es una medida estadística que ajusta el efecto del azar en la concordancia observada. Dicho índice presenta dificultades en su interpretación en situaciones de alta/baja prevalencia, por lo tanto se optó por la incorporación del Gwet's AC1 permitiendo una mejor evaluación del nivel de acuerdo. En la sección de resultados solo se reportará el Índice Gwet's AC1 para la lectura de las Tablas por tener mayor robustez científica ${ }^{79} 80$.

Se ha calculado el Intervalo de Confianza en todos los casos excepto cuando había pocos datos para estimar el error estándar.

No se ha calculado el Índice Kappa en los casos con menos de 30 comparaciones, debido a que a menor cantidad de casos mayor es la imprecisión de la estimación, y cuando se presentaba una única clasificación para un grupo de alimentos por parte de las GAPA o de los perfiles. 
No se ha calculado el Gwet's AC1 en los casos en que sólo una casilla está completa (es una constante) y cuando hay una combinación entre un número de muestra bajo y casillas vacías debido a que no se puede hacer el cálculo de las probabilidades para la interpretación probabilística.

La interpretación de los coeficientes se realizará mediante el procedimiento de estandarización sugerido por Gwet ${ }^{81}$, que consiste en una estimación probabilística del rango de la escala Landis y $\operatorname{Koch}^{82}$ (Tabla 1) que estandariza la interpretación de varios coeficientes y supera los problemas de interpretación dados por las dificultades de interpretación del Índice kappa antes citadas y de la variación estadística de la estimación relacionada con el grupo evaluado.

Tabla 2: Interpretación del nivel de acuerdo de Landis y Koch utilizado en las variables incluidas en el estudio

\begin{tabular}{|l|l|}
\hline Interpretación & Nivel de acuerdo \\
\hline$<0$ & Sin acuerdo \\
\hline $0.01-0.20$ & Insignificante \\
\hline $0.21-0.40$ & Discreto \\
\hline $0.41-0.60$ & Moderado \\
\hline $0.61-0.80$ & Sustancial \\
\hline $0.81-0.99$ & Casi perfecto \\
\hline
\end{tabular}

Fuente: Landis y Koch, 1977.

Los productos "Sin dato" se han descartado de la muestra para el análisis del nivel de acuerdo, esto conlleva como limitación una sobreestimación de los productos "alimitar". Sin embargo, el impacto sobre la estimación no es relevante porque: 1) la distribución de los productos "sin dato" es por azar, se distribuyen de forma aleatoria. Se debe principalmente a errores en el ingreso de la información de la etiqueta nutricional de los envases a la base de datos, por ser una foto ilegible o por error de tipeo. Por lo cual no se espera un error sistemático que modifique el índice de acuerdo; 2) el total de productos sin dato representa menos del $15 \%$ de la muestra, 3) el tamaño muestral es suficiente para lograr una estimación correcta.

Las GAPA y los perfiles de sistemas de etiquetado frontal de semáforo presentan 3 resultados de calidad nutricional, mientras que los perfiles de sistemas de etiquetado frontal de advertencias presentan 2 resultados posibles. Las Tablas 3 y 4 muestran los términos equivalentes de los resultados de las GAPA con los resultados de los perfiles nutricionales para la clasificación de la calidad nutricional de los alimentos a fin de poder realizar el análisis del nivel de acuerdo. 
Tabla 3: Equivalencia de la clasificación de la calidad nutricional de las GAPA y los Perfiles de sistemas e etiquetado frontal de semáforo

\begin{tabular}{|l|l|}
\hline GAPA & $\begin{array}{l}\text { Perfiles de sistemas de } \\
\text { semáforo }\end{array}$ \\
\hline A promover & A promover \\
\hline Consumo moderado & Consumo moderado \\
\hline A limitar & A limitar \\
\hline
\end{tabular}

Fuente: Elaboración propia

Tabla 4: Equivalencia de la clasificación de la calidad nutricional de las GAPA y los Perfiles de sistemas de etiquetado frontal de advertencias.

\begin{tabular}{|l|l|}
\hline GAPA & $\begin{array}{l}\text { Perfiles de sistemas de } \\
\text { advertencia }\end{array}$ \\
\hline $\begin{array}{l}\text { A promover y consumo } \\
\text { moderado }\end{array}$ & A promover \\
\hline A limitar & A limitar \\
\hline
\end{tabular}

Fuente: Elaboración propia 


\section{1- Resultados}

Los resultados del mapeo de perfiles nutricionales de la región de Latinoamérica se encuentran en el ANEXO II. Se incluyeron un total de seis perfiles nutricionales de sistemas de etiquetado frontal: los perfiles de Chile, Uruguay, Perú, y OPS desarrollados para sistemas de advertencias y los perfiles de Ecuador y Bolivia desarrollados para sistemas de semáforo.

A continuación se detallan los resultados del análisis de la calidad nutricional según las GAPA y los perfiles nutricionales incluidos en este análisis.

En primer lugar, se describe la clasificación de la muestra en grupos y categorías de alimentos. Luego, se presentan los resultados del análisis de la calidad nutricional de los perfiles nutricionales y su comparación con las GAPA en el total de la muestra, y por último a nivel de los grupos y categorías de alimentos.

Análisis descriptivo de la muestra total: Clasificación en grupos y categorías de alimentos

Se analizó la calidad nutricional según las GAPA y según los perfiles nutricionales en una muestra total de 1703 alimentos clasificados en 8 grupos y 48 categorías de alimentos (Tabla 1). Los grupos están conformados por legumbres, cereales, papa, pan y pastas ( $\mathrm{n}=185,10,9 \%)$; frutas y verduras $(n=53,3,1 \%)$; carnes y huevo $(n=138,8,1 \%)$; leche, yogur y quesos $(n=205$, $12 \%)$; aceite crudo, frutos secos y semillas $(n=8,0,5 \%)$; alimentos de consumo opcional $(\mathrm{n}=10161,62,3 \%)$; bebidas sin azúcar $(\mathrm{n}=42,2,5 \%)$; y comidas pre-elaboradas $(\mathrm{n}=11,0,6 \%)$.

Tabla 1: Clasificación en grupos y categorías de alimentos de la muestra total $(n=1703)$

\begin{tabular}{|c|c|c|c|}
\hline \multirow{2}{*}{ Grupo de alimento } & \multirow{2}{*}{$\begin{array}{c}\text { Categorías } \\
\text { de alimentos }\end{array}$} & \multicolumn{2}{|c|}{ Muestra total } \\
\hline & & $\mathrm{n}$ & $\%$ \\
\hline \multirow{10}{*}{$\begin{array}{l}\text { Legumbres, cereales, } \\
\text { papa, pan y pastas }\end{array}$} & Legumbres & 2 & $0,1 \%$ \\
\hline & Legumbres enlatadas & 7 & $0,4 \%$ \\
\hline & $\begin{array}{l}\text { Cereales refinados: } \\
\text { arroz, fideos, pastas }\end{array}$ & 37 & $2,2 \%$ \\
\hline & Cereales integrales & 15 & $0,9 \%$ \\
\hline & Pan blanco & 48 & $2,8 \%$ \\
\hline & Pan integral & 12 & $0,7 \%$ \\
\hline & Hortalizas feculentas & 2 & $0,1 \%$ \\
\hline & Pastas rellenas & 22 & $1,3 \%$ \\
\hline & $\begin{array}{c}\text { Premezclas panificados } \\
\text { y cereales }\end{array}$ & 12 & $0,7 \%$ \\
\hline & Amasados & 28 & $1,6 \%$ \\
\hline \multirow{3}{*}{ Frutas y verduras } & Frutas & 2 & $0,1 \%$ \\
\hline & Verduras & 10 & $0,6 \%$ \\
\hline & $\begin{array}{c}\text { Verduras y frutas } \\
\text { enlatadas }\end{array}$ & 25 & $1,5 \%$ \\
\hline
\end{tabular}




\begin{tabular}{|c|c|c|c|}
\hline & Verduras en salmuera & 13 & $0,8 \%$ \\
\hline & $\begin{array}{c}\text { Comidas listas de } \\
\text { verdura }\end{array}$ & 3 & $0,2 \%$ \\
\hline \multirow{4}{*}{ Carnes y huevo } & Carnes & 4 & $0,2 \%$ \\
\hline & Huevo & 1 & $0,1 \%$ \\
\hline & Carnes enlatadas & 16 & $0,9 \%$ \\
\hline & Carnes procesadas & 117 & $6,9 \%$ \\
\hline \multirow{6}{*}{ Leche, yogur y quesos } & Leche entera & 2 & $0,1 \%$ \\
\hline & $\begin{array}{l}\text { Leche parcialmente } \\
\text { descremada }\end{array}$ & 2 & $0,1 \%$ \\
\hline & Yogur entero & 7 & $0,4 \%$ \\
\hline & $\begin{array}{l}\text { Yogur parcialmente } \\
\text { descremado }\end{array}$ & 4 & $0,2 \%$ \\
\hline & $\begin{array}{l}\text { Queso duro, semiduro y } \\
\text { saborizados }\end{array}$ & 117 & $6,9 \%$ \\
\hline & Quesos blandos & 73 & $4,3 \%$ \\
\hline \multirow{3}{*}{$\begin{array}{l}\text { Aceite crudo, frutos } \\
\text { secos y semillas }\end{array}$} & Aceite & 2 & $0,1 \%$ \\
\hline & Frutos secos & 4 & $0,2 \%$ \\
\hline & Semillas & 2 & $0,1 \%$ \\
\hline \multirow{15}{*}{$\begin{array}{l}\text { Alimentos de consumo } \\
\text { opcional }\end{array}$} & Alimentos grasos & 38 & $2,2 \%$ \\
\hline & Azúcares libres & 3 & $0,2 \%$ \\
\hline & Alimentos dulces & 159 & $9,3 \%$ \\
\hline & Galletitas & 291 & $17,1 \%$ \\
\hline & Postres azucarados & 78 & $4,6 \%$ \\
\hline & Bebidas azucaradas & 101 & $5,9 \%$ \\
\hline & $\begin{array}{c}\text { Panificados dulces o } \\
\text { salados }\end{array}$ & 51 & $3,0 \%$ \\
\hline & $\begin{array}{c}\text { Premezclas panificados } \\
\text { dulces }\end{array}$ & 16 & $0,9 \%$ \\
\hline & $\begin{array}{l}\text { Barras de cereal con } \\
\text { azúcar }\end{array}$ & 12 & $0,7 \%$ \\
\hline & $\begin{array}{l}\text { Cereales de desayuno } \\
\text { con azúcar }\end{array}$ & 63 & $3,7 \%$ \\
\hline & $\begin{array}{l}\text { Snacks y productos de } \\
\text { copetin }\end{array}$ & 80 & $4,7 \%$ \\
\hline & Comidas rápidas & 55 & $3,2 \%$ \\
\hline & Aderezos & 57 & $3,3 \%$ \\
\hline & Salsas comerciales & 18 & $1,1 \%$ \\
\hline & $\begin{array}{l}\text { Caldos, saborizadores y } \\
\text { sopas comerciales }\end{array}$ & 39 & $2,3 \%$ \\
\hline
\end{tabular}




\begin{tabular}{|c|c|c|c|}
\multirow{4}{*}{ Bebidas sin azúcar } & Soda & 1 & $0,1 \%$ \\
\cline { 2 - 4 } & Aguas minerales & 7 & $0,4 \%$ \\
\cline { 2 - 4 } & Infusiones & 1 & $0,1 \%$ \\
\cline { 2 - 4 } & Bebidas edulcoradas & 33 & $1,9 \%$ \\
\hline \multirow{2}{*}{ Comidas pre-elaboradas } & Comidas pre-preparadas & 11 & $0,6 \%$ \\
\hline \multicolumn{2}{|c|}{ Total } & 1703 & $100,0 \%$ \\
\hline
\end{tabular}

Fuente: Elaboración propia

Análisis de la calidad nutricional en el total de la muestra

Nivel de exigencia de los perfiles respecto a las GAPA en el total de la muestra

Del total de la muestra, $71,2 \%(\mathrm{n}=1213)$ de los productos son clasificados como "a limitar" según las GAPA Estas poseen un nivel de exigencia mayor que el perfil de Ecuador (54,4\%, n=927), el perfil de Perú (52,9\%, n=901), y el perfil de Bolivia (51,3\%, n=874). Sin embargo, las GAPA tienen un nivel de exigencia menor en comparación con el perfil de OPS $(83,4 \%$, $\mathrm{n}=1421)$, Chile $(79,3 \%, \mathrm{n}=1350)$ y Uruguay $(76,3 \%, \mathrm{n}=1299)$, (Tabla 2$)$.

Tabla 2: Nivel de exigencia de las GAPA y los perfiles nutricionales seleccionados en el total de la muestra $(\mathrm{n}=1703)$

\begin{tabular}{|c|c|c|c|c|c|c|c|c|c|c|c|c|c|c|}
\hline & \multicolumn{2}{|c|}{$\begin{array}{l}\text { Clasificaci } \\
\text { ón GAPA }\end{array}$} & \multicolumn{2}{|c|}{$\begin{array}{l}\text { Clasificaci } \\
\text { ón OPS }\end{array}$} & \multicolumn{2}{|c|}{$\begin{array}{l}\text { Clasificaci } \\
\text { ón Uruguay }\end{array}$} & \multicolumn{2}{|c|}{$\begin{array}{l}\text { Clasificaci } \\
\text { ón Chile }\end{array}$} & \multicolumn{2}{|c|}{$\begin{array}{l}\text { Clasificaci } \\
\text { ón Perú }\end{array}$} & \multicolumn{2}{|c|}{$\begin{array}{l}\text { Clasificaci } \\
\text { ón Bolivia }\end{array}$} & \multicolumn{2}{|c|}{$\begin{array}{l}\text { Clasificaci } \\
\text { ón Ecuador }\end{array}$} \\
\hline & $\mathrm{n}$ & $\%$ & $\mathrm{n}$ & $\%$ & $\mathrm{n}$ & $\%$ & $\mathrm{n}$ & $\%$ & $\mathrm{n}$ & $\%$ & $\mathrm{n}$ & $\%$ & $\mathrm{n}$ & $\%$ \\
\hline $\begin{array}{l}\text { A } \\
\text { Promov } \\
\text { er }\end{array}$ & 490 & $\begin{array}{c}28,8 \\
\%\end{array}$ & 136 & $8,0 \%$ & 255 & $\begin{array}{c}15,0 \\
\%\end{array}$ & 284 & $\begin{array}{c}16,7 \\
\%\end{array}$ & 587 & $\begin{array}{c}34,5 \\
\%\end{array}$ & 89 & $5,2 \%$ & 76 & $4,5 \%$ \\
\hline $\begin{array}{l}\text { Consum } \\
\text { o } \\
\text { moderad } \\
\text { o } \\
\end{array}$ & $\mathrm{n} / \mathrm{c}$ & $\mathrm{n} / \mathrm{c}$ & $\mathrm{n} / \mathrm{c}$ & $\mathrm{n} / \mathrm{c}$ & $\mathrm{n} / \mathrm{c}$ & $\mathrm{n} / \mathrm{c}$ & $\mathrm{n} / \mathrm{c}$ & $\mathrm{n} / \mathrm{c}$ & $\mathrm{n} / \mathrm{c}$ & $\mathrm{n} / \mathrm{c}$ & 658 & $\begin{array}{c}38,6 \\
\%\end{array}$ & 576 & $\begin{array}{c}33,8 \\
\%\end{array}$ \\
\hline $\begin{array}{l}\text { A } \\
\text { Limitar }\end{array}$ & $\begin{array}{c}121 \\
3\end{array}$ & $\begin{array}{c}71,2 \\
\%\end{array}$ & $\begin{array}{c}142 \\
1\end{array}$ & $\begin{array}{c}83,4 \\
\%\end{array}$ & $\begin{array}{c}129 \\
9\end{array}$ & $\begin{array}{c}76,3 \\
\%\end{array}$ & $\begin{array}{c}135 \\
0\end{array}$ & $\begin{array}{c}79,3 \\
\%\end{array}$ & 901 & $\begin{array}{c}52,9 \\
\%\end{array}$ & 874 & $\begin{array}{c}51,3 \\
\%\end{array}$ & 927 & $\begin{array}{c}54,4 \\
\%\end{array}$ \\
\hline $\begin{array}{l}\text { Sin } \\
\text { dato* }\end{array}$ & 0 & $0,0 \%$ & 146 & $8,6 \%$ & 149 & $8,7 \%$ & 69 & $4,1 \%$ & 215 & $\begin{array}{c}12,6 \\
\%\end{array}$ & 82 & $4,8 \%$ & 124 & $7,3 \%$ \\
\hline Total & $\begin{array}{c}170 \\
3\end{array}$ & $\begin{array}{c}100 \\
\%\end{array}$ & $\begin{array}{c}170 \\
3\end{array}$ & $\begin{array}{c}100 \\
\%\end{array}$ & $\begin{array}{c}170 \\
3\end{array}$ & $\begin{array}{c}100 \\
\%\end{array}$ & $\begin{array}{c}170 \\
3 \\
\end{array}$ & $\begin{array}{c}100 \\
\%\end{array}$ & $\begin{array}{c}170 \\
3\end{array}$ & $\begin{array}{c}100 \\
\%\end{array}$ & $\begin{array}{c}170 \\
3 \\
\end{array}$ & $\begin{array}{c}100 \\
\%\end{array}$ & $\begin{array}{c}170 \\
3 \\
\end{array}$ & $\begin{array}{c}100 \\
\%\end{array}$ \\
\hline
\end{tabular}

* Alimentos que no pudieron clasificarse por falta de información.

Fuente: Elaboración propia

Nivel de exigencia por umbral de nutriente critico en los perfiles en el total de la muestra

La Tabla 3 muestra que el perfil de OPS y el perfil de Uruguay son los más exigentes de los perfiles nutricionales evaluados en todos los umbrales de nutrientes, con excepción del umbral de energía que no lo incluyen.

Al analizar los productos con cada perfil se pudo observar que en cuanto al umbral de sodio, el 48\% (n=813) de los alimentos lo supera según el perfil de OPS, el 34\% (n=582) según el 


\begin{tabular}{|c|c|c|c|c|c|c|c|c|c|c|c|}
\hline \multirow[t]{2}{*}{ Perfil } & \multicolumn{2}{|c|}{$\begin{array}{c}\text { Promov } \\
\text { er }\end{array}$} & \multicolumn{2}{|c|}{ moderado } & \multicolumn{2}{|c|}{ Limitar } & & \multirow[t]{2}{*}{ ón } & & & \multirow{2}{*}{$\begin{array}{c}\text { ón } \\
\text { probabilísti } \\
\text { ca }\end{array}$} \\
\hline & $\mathrm{n}$ & $\%$ & $\mathrm{n}$ & $\%$ & $\mathrm{n}$ & $\%$ & & & & & \\
\hline OPS & & & & & & & $\begin{array}{c}\% \\
\text { acuerdo }\end{array}$ & 77 & 74 & 79 & Sustancial \\
\hline A Promover & 118 & 26 & $\mathrm{n} / \mathrm{c}^{* *}$ & $\mathrm{n} / \mathrm{c}$ & 18 & 2 & Kappa & 0,284 & $\begin{array}{c}0,23 \\
3 \\
\end{array}$ & $\begin{array}{c}0,33 \\
5 \\
\end{array}$ & Discreto \\
\hline A Limitar & 344 & 74 & $\mathrm{n} / \mathrm{c}$ & $\mathrm{n} / \mathrm{c}$ & $\begin{array}{c}107 \\
7 \\
\end{array}$ & $\begin{array}{l}9 \\
8 \\
\end{array}$ & $\begin{array}{l}\text { Gwet's } \\
\text { AC1 }\end{array}$ & 0,663 & $\begin{array}{c}0,62 \\
6 \\
\end{array}$ & 0,7 & Sustancial \\
\hline Total & 462 & & & & $\begin{array}{c}109 \\
5 \\
\end{array}$ & & $\mathrm{n}=$ & \multicolumn{2}{|l|}{1557} & & \\
\hline Uruguay & & & & & & & $\begin{array}{c}\% \\
\text { acuerdo }\end{array}$ & 77 & 75 & 80 & Sustancial \\
\hline A Promover & 187 & 40 & $\mathrm{n} / \mathrm{c}$ & $\mathrm{n} / \mathrm{c}$ & 68 & 6 & Kappa & 0,367 & $\begin{array}{c}0,31 \\
0 \\
\end{array}$ & $\begin{array}{c}0,41 \\
6 \\
\end{array}$ & Discreto \\
\hline A Limitar & 286 & 60 & $\mathrm{n} / \mathrm{c}$ & $\mathrm{n} / \mathrm{c}$ & $\begin{array}{c}101 \\
3 \\
\end{array}$ & $\begin{array}{l}9 \\
4 \\
\end{array}$ & \begin{tabular}{|l} 
Gwet's \\
AC1 \\
\end{tabular} & 0,651 & $\begin{array}{c}0,61 \\
3 \\
\end{array}$ & $\begin{array}{c}0,68 \\
9 \\
\end{array}$ & Sustancial \\
\hline Total & 473 & & & & \begin{tabular}{|c|}
108 \\
1 \\
\end{tabular} & & $\mathrm{n}=$ & \multicolumn{2}{|l|}{1554} & & \\
\hline Chile & & & & & & & $\begin{array}{c}\% \\
\\
\text { acuerdo }\end{array}$ & 71 & 69 & 74 & Sustancial \\
\hline A Promover & 148 & 31 & $\mathrm{n} / \mathrm{c}$ & $\mathrm{n} / \mathrm{c}$ & 136 & $\begin{array}{l}1 \\
2 \\
\end{array}$ & Kappa & 0,211 & $\begin{array}{c}0,16 \\
0\end{array}$ & $\begin{array}{c}0,26 \\
3\end{array}$ & $\begin{array}{l}\text { Insignifica } \\
\text { nte }\end{array}$ \\
\hline A Limitar & 330 & 69 & $\mathrm{n} / \mathrm{c}$ & $\mathrm{n} / \mathrm{c}$ & $\begin{array}{c}102 \\
0 \\
\end{array}$ & $\begin{array}{l}8 \\
8 \\
\end{array}$ & $\begin{array}{l}\text { Gwet's } \\
\text { AC1 } \\
\end{array}$ & 0,558 & $\begin{array}{c}0,51 \\
7 \\
\end{array}$ & \begin{tabular}{|c|}
0,59 \\
9 \\
\end{tabular} & Moderado \\
\hline Total & 478 & & & & $\begin{array}{c}115 \\
6 \\
\end{array}$ & & $\mathrm{n}=$ & \multicolumn{2}{|l|}{1634} & & \\
\hline Perú & & & & & & & $\begin{array}{c}\% \\
\text { acuerdo } \\
\end{array}$ & 65 & 62 & 67 & Sustancial \\
\hline A Promover & 266 & 56 & $\mathrm{n} / \mathrm{c}$ & $\mathrm{n} / \mathrm{c}$ & 321 & $\begin{array}{l}3 \\
2 \\
\end{array}$ & Kappa & 0,223 & $\begin{array}{c}0,17 \\
2 \\
\end{array}$ & \begin{tabular}{|c|}
0,27 \\
5 \\
\end{tabular} & $\begin{array}{l}\text { Insignifica } \\
\text { nte } \\
\end{array}$ \\
\hline A Limitar & 205 & 44 & $\mathrm{n} / \mathrm{c}$ & $\mathrm{n} / \mathrm{c}$ & 696 & $\begin{array}{l}6 \\
8 \\
\end{array}$ & $\begin{array}{l}\text { Gwet's } \\
\text { AC1 }\end{array}$ & 0,373 & $\begin{array}{c}0,32 \\
4 \\
\end{array}$ & $\begin{array}{c}0,42 \\
2 \\
\end{array}$ & Discreto \\
\hline Total & 471 & & & & $\begin{array}{c}101 \\
7 \\
\end{array}$ & & $\mathrm{n}=$ & \multicolumn{2}{|l|}{1488} & & \\
\hline \multicolumn{12}{|l|}{ Ecuador } \\
\hline A Promover & 34 & 25 & 36 & 12 & 6 & 1 & $\begin{array}{c}\% \\
\text { acuerdo }\end{array}$ & 55 & 53 & 58 & Moderado \\
\hline $\begin{array}{l}\text { Consumo } \\
\text { moderado }\end{array}$ & 49 & 36 & 118 & 39 & 409 & $\begin{array}{l}3 \\
6 \\
\end{array}$ & Kappa & 0,117 & $\begin{array}{c}0,07 \\
6 \\
\end{array}$ & $\begin{array}{c}0,15 \\
8 \\
\end{array}$ & $\begin{array}{l}\text { Insignifica } \\
\text { nte }\end{array}$ \\
\hline A Limitar & 52 & 39 & 150 & 49 & 725 & $\begin{array}{l}6 \\
4 \\
\end{array}$ & $\begin{array}{l}\text { Gwet's } \\
\text { AC1 }\end{array}$ & 0,410 & $\begin{array}{c}0,37 \\
4 \\
\end{array}$ & $\begin{array}{c}0,44 \\
6 \\
\end{array}$ & Moderado \\
\hline Total & 135 & & 304 & & $\begin{array}{c}114 \\
0 \\
\end{array}$ & & $\mathrm{n}=$ & 1579 & & & \\
\hline \multicolumn{12}{|l|}{ Bolivia } \\
\hline A Promover & 37 & 27 & 45 & 14 & 7 & 1 & $\begin{array}{c}\% \\
\text { acuerdo }\end{array}$ & 51 & 49 & 54 & Moderado \\
\hline $\begin{array}{l}\text { Consumo } \\
\text { moderado }\end{array}$ & 48 & 35 & 133 & 40 & 477 & $\begin{array}{l}4 \\
2 \\
\end{array}$ & Kappa & 0,083 & $\begin{array}{c}0,04 \\
4 \\
\end{array}$ & \begin{tabular}{|c|}
0,12 \\
3 \\
\end{tabular} & $\begin{array}{l}\text { Insignifica } \\
\text { nte } \\
\end{array}$ \\
\hline A Limitar & 54 & 39 & 155 & 47 & 665 & $\begin{array}{l}5 \\
8 \\
\end{array}$ & $\begin{array}{l}\text { Gwet's } \\
\text { AC1 }\end{array}$ & 0,350 & $\begin{array}{c}0,31 \\
5 \\
\end{array}$ & $\begin{array}{c}0,38 \\
6 \\
\end{array}$ & Discreto \\
\hline Total & 139 & & 333 & & $\begin{array}{c}114 \\
9 \\
\end{array}$ & & $\mathrm{n}=$ & 1621 & & & \\
\hline
\end{tabular}

* En la lectura de la Tabla se reporta solo el Índice Gwet's AC1 por tener mayor robustez científica (Ver metodología)

**No corresponde. 
Fuente: Elaboración propia

Análisis de la calidad nutricional de cada perfil y su comparación con las GAPA por grupos y categorías de alimentos.

\section{Perfil OPS y GAPA}

Como se mencionó en el apartado anterior el Perfil de OPS tiene un nivel de acuerdo sustancial con las GAPA (Gwet's AC1=0,663) (Tabla 3). En la Tabla 5 se observa un mayor acuerdo en el grupo alimentos de consumo opcional con un acuerdo casi perfecto (Gwet's AC1= $0,920)$ y en el grupo carnes y huevos con un acuerdo sustancial (Gwet's $A C 1=0,847)$. Un menor acuerdo se encontró en el grupo de frutas y verduras con un acuerdo discreto (Gwet's AC1= 0,218), en el grupo de legumbres, cereales, papa, pan y pastas con un acuerdo insignificante (Gwet's $\mathrm{AC} 1=0,091)$ y en los grupos de leche, yogures y quesos (Gwet's $\mathrm{AC} 1=-0,923$ ) y bebidas sin azúcar (Gwet's $\mathrm{ACl}=-0,227)$ en donde no se encontró acuerdo. En los grupos donde no se pudo calcular el Índice Gwet's AC1 ni el Kappa, el porcentaje de acuerdo muestra un acuerdo insignificante en el grupo comidas pre-elaboradas y un acuerdo perfecto en el grupo aceite crudo, frutos secos y semillas.

Tabla 5: Nivel de acuerdo entre el perfil de OPS y las GAPA por grupos de alimentos $(\mathrm{n}=1557)$

\begin{tabular}{|c|c|c|c|c|c|c|c|c|c|c|c|}
\hline \multirow{3}{*}{$\begin{array}{l}\text { Grupo de } \\
\text { alimento }\end{array}$} & \multirow{3}{*}{$\begin{array}{l}\text { Muest } \\
\text { ra } \\
\text { total } \\
\text { (n) }\end{array}$} & \multirow{3}{*}{$\begin{array}{c}\text { Clasificac } \\
\text { ion Perfil } \\
\text { OPS }\end{array}$} & \multicolumn{4}{|c|}{ Clasificación GAPA } & \multicolumn{5}{|c|}{ Nivel de acuerdo* } \\
\hline & & & \multicolumn{2}{|c|}{$\begin{array}{c}\text { A } \\
\text { promover }\end{array}$} & \multicolumn{2}{|c|}{ A limitar } & \multirow{2}{*}{ Indicador } & \multirow{2}{*}{$\begin{array}{l}\text { Estimaci } \\
\text { ón }\end{array}$} & \multirow{2}{*}{\multicolumn{2}{|c|}{ IC 95\% }} & \multirow{2}{*}{$\begin{array}{c}\text { Interpretac } \\
\text { ión } \\
\text { probabilíst } \\
\text { ica }\end{array}$} \\
\hline & & & $\mathrm{N}$ & $\%$ & $\mathrm{~N}$ & $\%$ & & & & & \\
\hline \multirow{3}{*}{$\begin{array}{l}\text { Legumbres, } \\
\text { cereales, } \\
\text { papa, pan y } \\
\text { pastas }\end{array}$} & \multirow{3}{*}{179} & $\begin{array}{l}\text { A } \\
\text { promover }\end{array}$ & 70 & $44 \%$ & 0 & $0 \%$ & $\begin{array}{c}\% \\
\text { acuerdo }\end{array}$ & 51 & 43 & 58 & Moderado \\
\hline & & A limitar & 88 & $56 \%$ & 21 & $\begin{array}{c}100 \\
\% \\
\end{array}$ & Kappa & 0,157 & $\begin{array}{c}0,08 \\
8 \\
\end{array}$ & $\begin{array}{c}0,22 \\
6 \\
\end{array}$ & $\begin{array}{l}\text { Insignifica } \\
\text { nte }\end{array}$ \\
\hline & & & & & & & $\begin{array}{c}\text { Gwet's } \\
\text { ACl }\end{array}$ & 0,091 & $\begin{array}{c}- \\
0,07 \\
0\end{array}$ & $\begin{array}{c}0,25 \\
2\end{array}$ & $\begin{array}{l}\text { Insignifica } \\
\text { nte }\end{array}$ \\
\hline \multirow{3}{*}{$\begin{array}{l}\text { Frutas y } \\
\text { verduras }\end{array}$} & \multirow{3}{*}{53} & $\begin{array}{l}\text { A } \\
\text { promover }\end{array}$ & 19 & $48 \%$ & 0 & $0 \%$ & $\begin{array}{c}\% \\
\text { acuerdo }\end{array}$ & 60 & 47 & 74 & Moderado \\
\hline & & A limitar & 21 & $53 \%$ & 13 & $\begin{array}{c}100 \\
\% \\
\end{array}$ & Kappa & 0,307 & $\begin{array}{c}0,13 \\
6 \\
\end{array}$ & $\begin{array}{c}0,47 \\
9 \\
\end{array}$ & Discreto \\
\hline & & & & & & & $\begin{array}{l}\text { Gwet's } \\
\text { AC1 }\end{array}$ & 0,218 & $\begin{array}{c}- \\
0,05 \\
8\end{array}$ & $\begin{array}{c}0,49 \\
3\end{array}$ & Discreto \\
\hline \multirow{3}{*}{$\begin{array}{c}\text { Carnes y } \\
\text { huevo }\end{array}$} & \multirow{3}{*}{124} & $\begin{array}{l}\text { A } \\
\text { promover }\end{array}$ & 5 & $29 \%$ & 3 & $3 \%$ & $\begin{array}{c}\% \\
\text { acuerdo }\end{array}$ & 88 & 80 & 96 & $\begin{array}{l}\text { Casi } \\
\text { Perfecto }\end{array}$ \\
\hline & & A limitar & 12 & $71 \%$ & $\begin{array}{c}10 \\
4\end{array}$ & $97 \%$ & Kappa & 0,386 & $\begin{array}{c}0,14 \\
4\end{array}$ & $\begin{array}{c}0,62 \\
9\end{array}$ & Discreto \\
\hline & & & & & & & $\begin{array}{l}\text { Gwet's } \\
\text { AC1 }\end{array}$ & 0,847 & $\begin{array}{c}0,75 \\
1\end{array}$ & $\begin{array}{c}0,94 \\
3\end{array}$ & $\begin{array}{l}\text { Casi } \\
\text { Perfecto }\end{array}$ \\
\hline \multirow{2}{*}{$\begin{array}{c}\text { Leche, yogur } \\
\text { y quesos }\end{array}$} & \multirow{2}{*}{193} & $\begin{array}{l}\text { A } \\
\text { promover }\end{array}$ & 6 & $3 \%$ & 0 & $\begin{array}{c}\mathrm{X}^{* *} \\
*\end{array}$ & $\begin{array}{c}\% \\
\text { acuerdo }\end{array}$ & 3 & 0 & 6 & $\begin{array}{l}\text { Insignifica } \\
\text { nte }\end{array}$ \\
\hline & & A limitar & $\begin{array}{c}18 \\
7 \\
\end{array}$ & $97 \%$ & 0 & $\mathrm{x}$ & Kappa & $\mathrm{x}$ & $\mathrm{x}$ & $\mathrm{x}$ & $\mathrm{x}$ \\
\hline
\end{tabular}




\begin{tabular}{|c|c|c|c|c|c|c|c|c|c|c|c|}
\hline & & & & & & & $\begin{array}{l}\text { Gwet's } \\
\text { AC1 }\end{array}$ & $-0,923$ & $\begin{array}{c}- \\
0,99 \\
4\end{array}$ & 0,85 & $\begin{array}{l}\text { Sin } \\
\text { Acuerdo }\end{array}$ \\
\hline \multirow{3}{*}{$\begin{array}{c}\text { Aceite } \\
\text { crudo, frutos } \\
\text { secos y } \\
\text { semillas }\end{array}$} & \multirow{3}{*}{8} & $\begin{array}{l}\text { A } \\
\text { promover }\end{array}$ & 8 & $\begin{array}{c}100 \\
\%\end{array}$ & 0 & $\mathrm{x}$ & $\begin{array}{c}\% \\
\text { acuerdo }\end{array}$ & 100 & $\mathrm{x}$ & $\mathrm{x}$ & Perfecto \\
\hline & & A limitar & 0 & $0 \%$ & 0 & $\mathrm{x}$ & Kappa & $\mathrm{x}$ & $\mathrm{x}$ & $\mathrm{x}$ & $\mathrm{x}$ \\
\hline & & & & & & & $\begin{array}{c}\text { Gwet's } \\
\text { AC1 }\end{array}$ & $\mathrm{x}$ & $\mathrm{x}$ & $\mathrm{x}$ & $\mathrm{x}$ \\
\hline \multirow{3}{*}{$\begin{array}{l}\text { Alimentos } \\
\text { de consumo } \\
\text { opcional }\end{array}$} & \multirow{3}{*}{954} & $\begin{array}{l}\text { A } \\
\text { promover }\end{array}$ & 0 & $\mathrm{x}$ & 15 & $2 \%$ & $\begin{array}{c}\% \\
\text { acuerdo }\end{array}$ & 98 & 96 & 100 & $\begin{array}{l}\text { Casi } \\
\text { Perfecto }\end{array}$ \\
\hline & & A limitar & 0 & $\mathrm{x}$ & $\begin{array}{c}93 \\
9\end{array}$ & $98 \%$ & Kappa & $\mathrm{x}$ & $\mathrm{x}$ & $\mathrm{x}$ & $\mathrm{x}$ \\
\hline & & & & & & & $\begin{array}{c}\text { Gwet's } \\
\text { AC1 }\end{array}$ & 0,984 & $\begin{array}{c}0,96 \\
3\end{array}$ & 1.00 & $\begin{array}{l}\text { Casi } \\
\text { Perfecto }\end{array}$ \\
\hline \multirow{3}{*}{$\begin{array}{l}\text { Bebidas sin } \\
\text { azúcar }\end{array}$} & \multirow{3}{*}{35} & $\begin{array}{l}\text { A } \\
\text { promover }\end{array}$ & 10 & $29 \%$ & 0 & $\mathrm{x}$ & $\begin{array}{c}\% \\
\text { acuerdo }\end{array}$ & 29 & 12 & 45 & Discreto \\
\hline & & A limitar & 25 & $71 \%$ & 0 & $\mathrm{x}$ & Kappa & $\mathrm{x}$ & $\mathrm{x}$ & $\mathrm{x}$ & $\mathrm{x}$ \\
\hline & & & & & & & $\begin{array}{c}\text { Gwet's } \\
\text { AC1 }\end{array}$ & $-0,227$ & $\begin{array}{c}- \\
0,62 \\
1\end{array}$ & $\begin{array}{c}0,16 \\
6\end{array}$ & $\begin{array}{l}\text { Sin } \\
\text { Acuerdo }\end{array}$ \\
\hline \multirow{3}{*}{$\begin{array}{l}\text { Comidas } \\
\text { pre- } \\
\text { elaboradas }\end{array}$} & \multirow{3}{*}{11} & $\begin{array}{l}\text { A } \\
\text { promover }\end{array}$ & 0 & $0 \%$ & 0 & $\mathrm{x}$ & $\begin{array}{c}\% \\
\text { acuerdo }\end{array}$ & 0 & $\mathrm{x}$ & $\mathrm{x}$ & $\begin{array}{l}\text { Sin } \\
\text { acuerdo }\end{array}$ \\
\hline & & A limitar & 11 & $\begin{array}{c}100 \\
\%\end{array}$ & 0 & $\mathrm{x}$ & Kappa & $\mathrm{x}$ & $\mathrm{x}$ & $\mathrm{x}$ & $\mathrm{x}$ \\
\hline & & & & & & & $\begin{array}{c}\text { Gwet's } \\
\text { AC1 }\end{array}$ & $\mathrm{x}$ & $\mathrm{x}$ & $\mathrm{x}$ & $\mathrm{x}$ \\
\hline $\begin{array}{l}\text { Muestra } \\
\text { Total** }\end{array}$ & 1557 & & & & & & & & & & \\
\hline
\end{tabular}

* En la lectura de la Tabla se reporta solo el Índice Gwet's AC1 por tener mayor robustez científica (Ver metodología)

** Los productos de la categoría sin dato se han descartado de la muestra total para el análisis del nivel de acuerdo (Ver metodología)

***No se ha podido realizar el cálculo por la naturaleza de los datos.

Fuente: Elaboración Propia

En cuanto al nivel de exigencia, las GAPA poseen un nivel de exigencia menor que el perfil de OPS (71,2\%vs $83,4 \%$ ) (Tabla 2). En la Tabla 6 se observa que a nivel de los grupos de alimentos, las GAPA son menos exigentes que el perfil de OPS en todos los grupos excepto en el grupo carnes y huevos (87\% GAPA vs $84,1 \%$ OPS), en el grupo alimentos de consumo opcional (100\% GAPA vs $88,5 \%$ OPS), y en el grupo de aceite crudo, frutos secos y semillas ( $0 \%$ GAPA y $0 \%$ OPS).

En el grupo de legumbres, cereales, papa, pan y pastas, el perfil de OPS coincide con las GAPA en limitar la mayoría de las pastas rellenas $(95,5 \%)$ por su alto contenido en sodio y grasas totales, saturadas y trans pero además limita productos de las siguientes categorías: $100 \%$ legumbres enlatadas y $8,1 \%$ cereales refinados por su alto contenido de sodio, $81,3 \%$ pan blanco y $83,3 \%$ pan integral por su alto contenido en sodio y/o azúcares y $66,7 \%$ premezclas de panificados y cereales, y $75 \%$ amasados por su alto contenido en sodio y grasas totales, grasas saturadas, y/o trans. (Tabla 1 ANEXO III y Tabla 6) 
En el grupo de frutas y verduras el perfil de OPS coincide con las GAPA en limitar todas las verduras en salmuera pero además limita productos de las siguientes categorías: $72 \%$ de verduras y frutas enlatadas por el alto contenido de sodio, grasas totales, saturadas y/o trans y $100 \%$ de las comidas listas de verdura por el alto contenido en sodio y/o azúcares. (Tabla 1 ANEXO III y Tabla 6)

En el grupo de leche, yogur y quesos, difieren en la clasificación de las categorías de quesos, las GAPA promueven su consumo mientras que el perfil de OPS clasifica a limitar a un $95 \%$ de los quesos duros, semiduros y saborizados y un $89 \%$ de los quesos blandos, ambos por su alto contenido en sodio, grasas saturadas, grasas totales y grasas trans (Tabla 1 ANEXO III y Tabla 6)

En el grupo de bebidas sin azúcar las GAPA promueven su consumo mientras que el perfil de OPS lo clasifica como un grupo "a limitar" en un 59,5\% de los productos pertenecientes a la categoría de bebidas edulcoradas por tener un alto contenido de sodio y en azúcar (Tabla 1 ANEXO III y Tabla 6).

En comidas pre-elaboradas el perfil de OPS difiere con las GAPA en la clasificación de todos los productos incluidos, clasificándolos "a limitar" por su alto contenido en grasas saturadas, grasas totales, grasas trans y/o sodio (Tabla 1 ANEXO III y Tabla 6).

Tabla 6: Nivel de exigencia del perfil de OPS y las GAPA por grupos y por categorías de alimentos $(\mathrm{n}=1703)$

\begin{tabular}{|c|c|c|c|c|c|c|c|c|}
\hline \multirow[t]{2}{*}{ Grupo de alimento } & \multirow[t]{2}{*}{$\begin{array}{l}\text { Categoría de } \\
\text { alimentos }\end{array}$} & \multirow[t]{2}{*}{$\mathrm{n}$} & & \multicolumn{2}{|c|}{$\begin{array}{l}\text { Clasificación } \\
\text { GAPA }\end{array}$} & \multicolumn{3}{|c|}{ Clasificación Perfil OPS } \\
\hline & & & & $\begin{array}{c}\text { A } \\
\text { Promover }\end{array}$ & $\begin{array}{c}\text { A } \\
\text { Limitar }\end{array}$ & $\begin{array}{c}\text { A } \\
\text { Promover }\end{array}$ & $\begin{array}{c}\text { A } \\
\text { Limitar }\end{array}$ & $\begin{array}{c}\text { Sin } \\
\text { dato* }\end{array}$ \\
\hline \multirow{17}{*}{$\begin{array}{c}\text { Legumbres, } \\
\text { cereales, papa, pan } \\
\text { y pastas }\end{array}$} & \multirow[t]{2}{*}{ Legumbres } & \multirow[t]{2}{*}{2} & $\mathrm{n}$ & 2 & 0 & 2 & 0 & 0 \\
\hline & & & $\%$ & $100,0 \%$ & $0,0 \%$ & $100,0 \%$ & $0,0 \%$ & $0,0 \%$ \\
\hline & \multirow{2}{*}{$\begin{array}{l}\text { Legumbres } \\
\text { enlatadas }\end{array}$} & \multirow[t]{2}{*}{7} & $\mathrm{n}$ & 7 & 0 & 0 & 7 & 0 \\
\hline & & & $\%$ & $100,0 \%$ & $0,0 \%$ & $0,0 \%$ & $100,0 \%$ & $0,0 \%$ \\
\hline & \multirow{2}{*}{$\begin{array}{l}\text { Cereales refinados: } \\
\text { arroz, fideos, pastas }\end{array}$} & \multirow[t]{2}{*}{37} & $\mathrm{n}$ & 37 & 0 & 33 & 3 & 1 \\
\hline & & & $\%$ & $100,0 \%$ & $0,0 \%$ & $89,2 \%$ & $8,1 \%$ & $2,7 \%$ \\
\hline & \multirow[t]{2}{*}{ Cereales integrales } & \multirow[t]{2}{*}{15} & $\mathrm{n}$ & 15 & 0 & 15 & 0 & 0 \\
\hline & & & $\%$ & $100,0 \%$ & $0,0 \%$ & $100,0 \%$ & $0,0 \%$ & $0,0 \%$ \\
\hline & \multirow[t]{2}{*}{ Pan blanco } & \multirow[t]{2}{*}{48} & $\mathrm{n}$ & 48 & 0 & 9 & 39 & 0 \\
\hline & & & $\%$ & $100,0 \%$ & $0,0 \%$ & $18,8 \%$ & $81,3 \%$ & $0,0 \%$ \\
\hline & \multirow{2}{*}{ Pan integral } & \multirow[t]{2}{*}{12} & $\mathrm{n}$ & 12 & 0 & 1 & 10 & 1 \\
\hline & & & $\%$ & $100,0 \%$ & $0,0 \%$ & $8,3 \%$ & $83,3 \%$ & $8,3 \%$ \\
\hline & \multirow{2}{*}{$\begin{array}{l}\text { Hortalizas } \\
\text { feculentas }\end{array}$} & \multirow[t]{2}{*}{2} & $\mathrm{n}$ & 2 & 0 & 2 & 0 & 0 \\
\hline & & & $\%$ & $100,0 \%$ & $0,0 \%$ & $100,0 \%$ & $0,0 \%$ & $0,0 \%$ \\
\hline & \multirow[t]{2}{*}{ Pastas rellenas } & \multirow[t]{2}{*}{22} & $\mathrm{n}$ & 0 & 22 & 0 & 21 & 1 \\
\hline & & & $\%$ & $0,0 \%$ & $100,0 \%$ & $0,0 \%$ & $95,5 \%$ & $4,5 \%$ \\
\hline & Premezclas & 12 & $\mathrm{n}$ & 12 & 0 & 4 & 8 & 0 \\
\hline
\end{tabular}




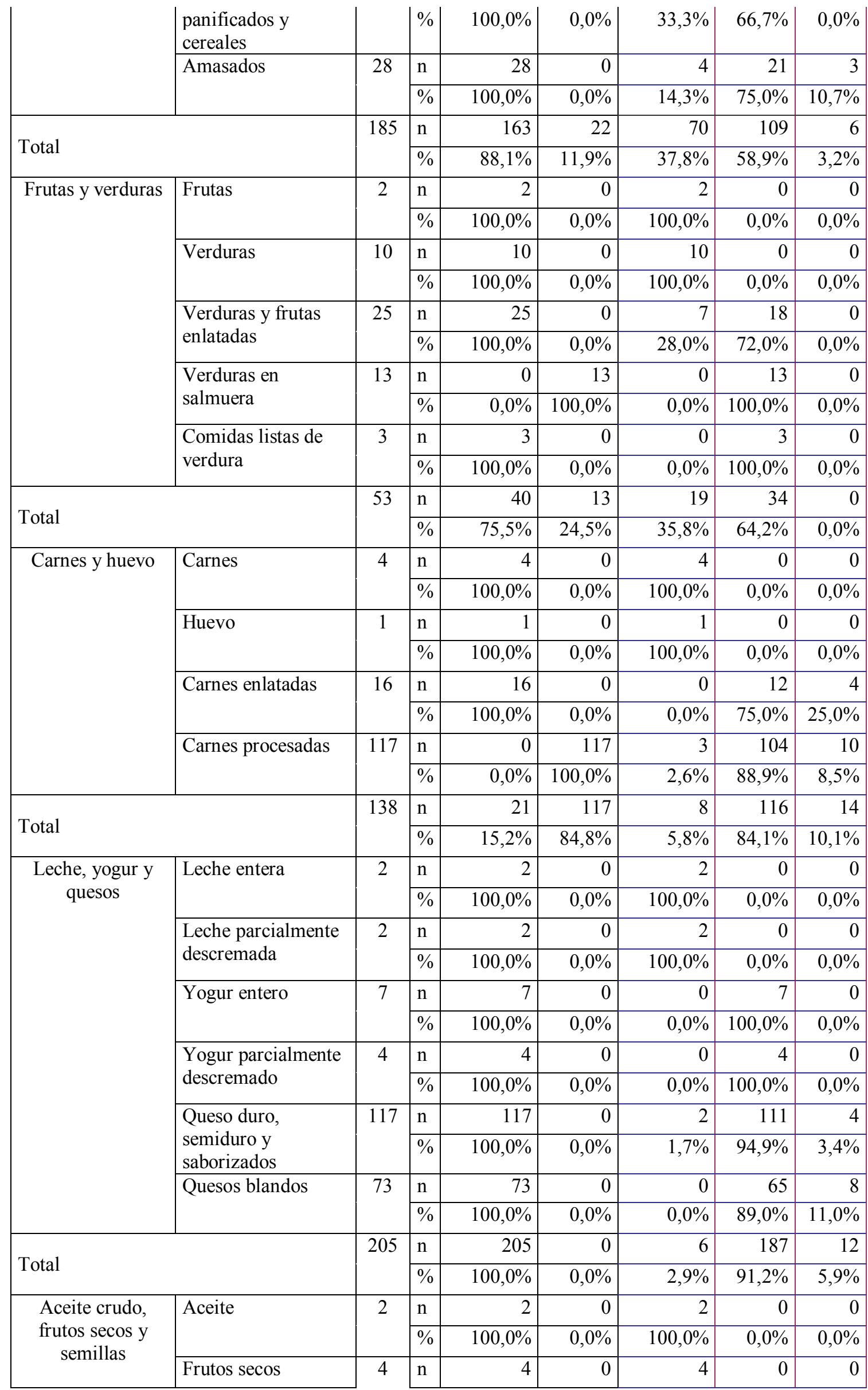




\begin{tabular}{|c|c|c|c|c|c|c|c|c|}
\hline & & & $\%$ & $100,0 \%$ & $0,0 \%$ & $100,0 \%$ & $0,0 \%$ & $0,0 \%$ \\
\hline & \multirow[t]{2}{*}{ Semillas } & \multirow[t]{2}{*}{2} & $\mathrm{n}$ & 2 & 0 & 2 & 0 & 0 \\
\hline & & & $\%$ & $100,0 \%$ & $0,0 \%$ & $100,0 \%$ & $0,0 \%$ & $0,0 \%$ \\
\hline \multirow{2}{*}{\multicolumn{2}{|c|}{ Total }} & \multirow[t]{2}{*}{8} & $\mathrm{n}$ & 8 & 0 & 8 & 0 & 0 \\
\hline & & & $\%$ & $100,0 \%$ & $0,0 \%$ & $100,0 \%$ & $0,0 \%$ & $0,0 \%$ \\
\hline \multirow{30}{*}{$\begin{array}{c}\text { Alimentos de } \\
\text { consumo opcional }\end{array}$} & \multirow[t]{2}{*}{ Alimentos grasos } & \multirow[t]{2}{*}{38} & $\mathrm{n}$ & 0 & 38 & 1 & 36 & 1 \\
\hline & & & $\%$ & $0,0 \%$ & $100,0 \%$ & $2,6 \%$ & $94,7 \%$ & $2,6 \%$ \\
\hline & \multirow[t]{2}{*}{ Azúcares libres } & \multirow[t]{2}{*}{3} & $\mathrm{n}$ & 0 & 3 & 1 & 2 & 0 \\
\hline & & & $\%$ & $0,0 \%$ & $100,0 \%$ & $33,3 \%$ & $66,7 \%$ & $0,0 \%$ \\
\hline & \multirow[t]{2}{*}{ Alimentos dulces } & \multirow[t]{2}{*}{159} & $\mathrm{n}$ & 0 & 159 & 2 & 146 & 11 \\
\hline & & & $\%$ & $0,0 \%$ & $100,0 \%$ & $1,3 \%$ & $91,8 \%$ & $6,9 \%$ \\
\hline & \multirow[t]{2}{*}{ Galletitas } & \multirow[t]{2}{*}{291} & $\mathrm{n}$ & 0 & 291 & 4 & 263 & 24 \\
\hline & & & $\%$ & $0,0 \%$ & $100,0 \%$ & $1,4 \%$ & $90,4 \%$ & $8,2 \%$ \\
\hline & \multirow{2}{*}{ Postres azucarados } & \multirow[t]{2}{*}{78} & $\mathrm{n}$ & 0 & 78 & 0 & 69 & 9 \\
\hline & & & $\%$ & $0,0 \%$ & $100,0 \%$ & $0,0 \%$ & $88,5 \%$ & $11,5 \%$ \\
\hline & \multirow[t]{2}{*}{ Bebidas azucaradas } & \multirow[t]{2}{*}{101} & $\mathrm{n}$ & 0 & 101 & 0 & 100 & 1 \\
\hline & & & $\%$ & $0,0 \%$ & $100,0 \%$ & $0,0 \%$ & $99,0 \%$ & $1,0 \%$ \\
\hline & \multirow{2}{*}{$\begin{array}{l}\text { Panificados dulces o } \\
\text { salados }\end{array}$} & \multirow[t]{2}{*}{51} & $\mathrm{n}$ & 0 & 51 & 0 & 40 & 11 \\
\hline & & & $\%$ & $0,0 \%$ & $100,0 \%$ & $0,0 \%$ & $78,4 \%$ & $21,6 \%$ \\
\hline & \multirow{2}{*}{$\begin{array}{l}\text { Premezclas } \\
\text { panificados dulces }\end{array}$} & \multirow[t]{2}{*}{16} & $\mathrm{n}$ & 0 & 16 & 0 & 11 & 5 \\
\hline & & & $\%$ & $0,0 \%$ & $100,0 \%$ & $0,0 \%$ & $68,8 \%$ & $31,3 \%$ \\
\hline & \multirow{2}{*}{$\begin{array}{l}\text { Barras de cereal con } \\
\text { azúcar }\end{array}$} & 12 & $\mathrm{n}$ & 0 & 12 & 0 & 9 & 3 \\
\hline & & & $\%$ & $0,0 \%$ & $100,0 \%$ & $0,0 \%$ & $75,0 \%$ & $25,0 \%$ \\
\hline & Cereales de & 63 & $\mathrm{n}$ & 0 & 63 & 0 & 45 & 18 \\
\hline & desayuno con azúcar & & $\%$ & $0,0 \%$ & $100,0 \%$ & $0,0 \%$ & $71,4 \%$ & $28,6 \%$ \\
\hline & Snacks y productos & 80 & $\mathrm{n}$ & 0 & 80 & 1 & 74 & 5 \\
\hline & & & $\%$ & $0,0 \%$ & $100,0 \%$ & $1,3 \%$ & $92,5 \%$ & $6,3 \%$ \\
\hline & Comidas rápidas & 55 & $\mathrm{n}$ & 0 & 55 & 5 & 48 & 2 \\
\hline & & & $\%$ & $0,0 \%$ & $100,0 \%$ & $9,1 \%$ & $87,3 \%$ & $3,6 \%$ \\
\hline & Aderezos & 57 & $\mathrm{n}$ & 0 & 57 & 0 & 44 & 13 \\
\hline & & & $\%$ & $0,0 \%$ & $100,0 \%$ & $0,0 \%$ & $77,2 \%$ & $22,8 \%$ \\
\hline & Salsas comerciales & 18 & $\mathrm{n}$ & 0 & 18 & 0 & 16 & 2 \\
\hline & & & $\%$ & $0,0 \%$ & $100,0 \%$ & $0,0 \%$ & $88,9 \%$ & $11,1 \%$ \\
\hline & Caldos, & 39 & $\mathrm{n}$ & 0 & 39 & 1 & 36 & 2 \\
\hline & $\begin{array}{l}\text { saborizadores y } \\
\text { sopas comerciales }\end{array}$ & & $\%$ & $0,0 \%$ & $100,0 \%$ & $2,6 \%$ & $92,3 \%$ & $5,1 \%$ \\
\hline & & 1061 & $\mathrm{n}$ & 0 & 1061 & 15 & 939 & 107 \\
\hline Total & & & $\%$ & $0,0 \%$ & $100,0 \%$ & $1,4 \%$ & $88,5 \%$ & $10,1 \%$ \\
\hline Bebidas sin azúcar & Soda & 1 & $\mathrm{n}$ & 1 & 0 & 1 & 0 & 0 \\
\hline & & & $\%$ & $100,0 \%$ & $0,0 \%$ & $100,0 \%$ & $0,0 \%$ & $0,0 \%$ \\
\hline & Aguas minerales & 7 & $\mathrm{n}$ & 7 & 0 & 7 & 0 & 0 \\
\hline & & & $\%$ & $100,0 \%$ & $0,0 \%$ & $100,0 \%$ & $0,0 \%$ & $0,0 \%$ \\
\hline & Infusiones & 1 & $\mathrm{n}$ & 1 & 0 & 1 & 0 & 0 \\
\hline & & & $\%$ & $100,0 \%$ & $0,0 \%$ & $100,0 \%$ & $0,0 \%$ & $0,0 \%$ \\
\hline & Bebidas edulcoradas & 33 & $\mathrm{n}$ & 33 & 0 & 1 & 25 & 7 \\
\hline
\end{tabular}




\begin{tabular}{|l|l|l|r|r|r|r|r|r|} 
& & $\%$ & $100,0 \%$ & $0,0 \%$ & $3,0 \%$ & $75,8 \%$ & $21,2 \%$ \\
\hline \multirow{2}{*}{ Total } & 42 & $\mathrm{n}$ & 42 & 0 & 10 & 25 & 7 \\
\cline { 3 - 9 } & & $\%$ & $100,0 \%$ & $0,0 \%$ & $23,8 \%$ & $59,5 \%$ & $16,7 \%$ \\
\hline Comidas pre-elaboradas & 11 & $\mathrm{n}$ & 11 & 0 & 0 & 11 & 0 \\
\cline { 2 - 8 } & & $\%$ & $100,0 \%$ & $0,0 \%$ & $0,0 \%$ & $100,0 \%$ & $0,0 \%$ \\
\hline Muestra Total & 1703 & $\mathrm{n}$ & 490 & 1213 & 136 & 1421 & 146 \\
\hline
\end{tabular}

* Alimentos que no pudieron clasificarse por falta de información.

Fuente: Elaboración Propia.

\section{Perfil Uruguay y GAPA}

Como se mencionó en el apartado anterior el Perfil de Uruguay tiene un nivel de acuerdo sustancial con las GAPA (Gwet's AC1=0,651) (Tabla 3). La Tabla 7 muestra que el perfil de Uruguay presentó un acuerdo casi perfecto con las GAPA en el grupo de alimentos de consumo opcional (Gwet's $\mathrm{AC} 1=0,936)$ y en el de carnes y huevos (Gwet's $\mathrm{AC} 1=0,894)$. En el grupo de frutas y verduras (Gwet's $\mathrm{AC} 1=0,7)$ y en el grupo bebidas sin azúcar (Gwet's $\mathrm{AC} 1=0,628$ ) presenta un acuerdo sustancial. Los grupos con menor acuerdo son el de legumbres, cereales, papa, pan y pastas con un acuerdo discreto (Gwet's $\mathrm{AC} 1=0,370$ ) y el de leche, yogures y quesos donde no se encontró acuerdo (Gwet's AC1=-0,819). En los grupos donde no se pudo calcular el Índice Gwet's AC1 ni el Kappa, el porcentaje de acuerdo muestra un acuerdo insignificante en el grupo comidas pre-elaboradas y un acuerdo perfecto en el grupo aceite crudo, frutos secos y semillas.

Tabla 7: Nivel de acuerdo entre el perfil de Uruguay y las GAPA por grupos de alimentos $(\mathrm{n}=1554)$

\begin{tabular}{|c|c|c|c|c|c|c|c|c|c|c|c|}
\hline \multirow{3}{*}{$\begin{array}{l}\text { Grupo de } \\
\text { alimento }\end{array}$} & \multirow{3}{*}{$\mathrm{n}$} & \multirow{3}{*}{$\begin{array}{c}\text { Clasificaci } \\
\text { on Perfil } \\
\text { Uruguay }\end{array}$} & \multicolumn{4}{|c|}{ Clasificación GAPA } & \multicolumn{5}{|c|}{ Nivel de acuerdo* } \\
\hline & & & \multicolumn{2}{|c|}{$\begin{array}{c}\text { A } \\
\text { promover }\end{array}$} & \multicolumn{2}{|c|}{ A limitar } & \multirow{2}{*}{ Indicador } & \multirow{2}{*}{$\begin{array}{l}\text { Estimaci } \\
\text { ón }\end{array}$} & \multirow{2}{*}{\multicolumn{2}{|c|}{ IC 95\% }} & \multirow{2}{*}{$\begin{array}{l}\text { Interpretaci } \\
\text { ón } \\
\text { probabilísti } \\
\text { ca }\end{array}$} \\
\hline & & & $\mathrm{n}$ & $\%$ & $\mathrm{n}$ & $\%$ & & & & & \\
\hline \multirow{3}{*}{$\begin{array}{c}\text { Legumbres, } \\
\text { cereales, } \\
\text { papa, pan y } \\
\text { pastas }\end{array}$} & \multirow{3}{*}{182} & $\begin{array}{l}\text { A } \\
\text { promover }\end{array}$ & 99 & $61 \%$ & 8 & $38 \%$ & $\begin{array}{c}\% \\
\text { acuerdo }\end{array}$ & 62 & 54 & 69 & Sustancial \\
\hline & & A limitar & 62 & $39 \%$ & 13 & $62 \%$ & Карра & 0,112 & $\begin{array}{c}0,00 \\
1\end{array}$ & $\begin{array}{c}0,22 \\
3 \\
\end{array}$ & $\begin{array}{l}\text { Insignifica } \\
\text { nte }\end{array}$ \\
\hline & & & & & & & $\begin{array}{c}\text { Gwet's } \\
\mathrm{ACl}\end{array}$ & 0,370 & $\begin{array}{c}0,22 \\
2\end{array}$ & $\begin{array}{c}0,51 \\
8\end{array}$ & iscreto \\
\hline \multirow{3}{*}{$\begin{array}{l}\text { Frutas y } \\
\text { verduras }\end{array}$} & \multirow{3}{*}{53} & \begin{tabular}{|l|} 
A \\
promover \\
\end{tabular} & 31 & $78 \%$ & 0 & $0 \%$ & $\begin{array}{c}\% \\
\text { acuerdo }\end{array}$ & 83 & 73 & 93 & $\begin{array}{l}\text { Casi } \\
\text { Perfecto }\end{array}$ \\
\hline & & A limitar & 9 & $23 \%$ & 13 & $\begin{array}{c}100 \\
\%\end{array}$ & Kappa & 0,628 & $\begin{array}{c}0,41 \\
6\end{array}$ & $\begin{array}{c}0,84 \\
1 \\
\end{array}$ & Sustancial \\
\hline & & & & & & & $\begin{array}{c}\text { Gwet's } \\
\text { AC1 }\end{array}$ & 0,700 & $\begin{array}{c}0,50 \\
0\end{array}$ & $\begin{array}{c}0,89 \\
5\end{array}$ & Sustancial \\
\hline \multirow{3}{*}{$\begin{array}{l}\text { Carnes y } \\
\text { huevo }\end{array}$} & \multirow{3}{*}{135} & $\begin{array}{l}\text { A } \\
\text { promover }\end{array}$ & 12 & $60 \%$ & 3 & $3 \%$ & $\begin{array}{c}\% \\
\text { acuerdo }\end{array}$ & 92 & 87 & 97 & $\begin{array}{l}\text { Casi } \\
\text { Perfecto }\end{array}$ \\
\hline & & A limitar & 8 & $40 \%$ & $\begin{array}{c}11 \\
2 \\
\end{array}$ & $97 \%$ & Kappa & 0,645 & $\begin{array}{c}0,45 \\
0\end{array}$ & $\begin{array}{c}0,84 \\
0 \\
\end{array}$ & Sustancial \\
\hline & & & & & & & $\begin{array}{c}\text { Gwet's } \\
\text { AC1 }\end{array}$ & 0,894 & $\begin{array}{c}0,82 \\
4\end{array}$ & $\begin{array}{c}0,96 \\
3 \\
\end{array}$ & $\begin{array}{l}\text { Casi } \\
\text { Perfecto }\end{array}$ \\
\hline
\end{tabular}




\begin{tabular}{|c|c|c|c|c|c|c|c|c|c|c|c|}
\hline \multirow{3}{*}{$\begin{array}{l}\text { Leche, yogur } \\
\text { y quesos }\end{array}$} & \multirow{3}{*}{199} & $\begin{array}{l}\text { A } \\
\text { promover }\end{array}$ & 12 & $6 \%$ & 0 & $\begin{array}{c}\mathrm{x}^{* *} \\
*\end{array}$ & $\begin{array}{c}\% \\
\text { acuerdo }\end{array}$ & 6 & 3 & 9 & $\begin{array}{l}\text { Insignifica } \\
\text { nte }\end{array}$ \\
\hline & & A limitar & 187 & $94 \%$ & 0 & $\mathrm{x}$ & Карра & 0 & 0 & 0 & $\begin{array}{l}\text { Sin } \\
\text { Acuerdo }\end{array}$ \\
\hline & & & & & & & $\begin{array}{c}\text { Gwet's } \\
\text { AC1 }\end{array}$ & $-0,865$ & $\begin{array}{c}- \\
0,94 \\
7\end{array}$ & $\begin{array}{c}- \\
0,78 \\
3\end{array}$ & $\begin{array}{l}\text { Sin } \\
\text { Acuerdo }\end{array}$ \\
\hline \multirow{3}{*}{$\begin{array}{l}\text { Aceite crudo, } \\
\text { frutos secos } \\
\text { y semillas }\end{array}$} & \multirow{3}{*}{8} & $\begin{array}{l}\text { A } \\
\text { promover }\end{array}$ & 8 & \#\#\# & 0 & $\mathrm{x}$ & $\begin{array}{c}\% \\
\text { acuerdo }\end{array}$ & 100 & $\mathrm{x}$ & $\mathrm{x}$ & Perfecto \\
\hline & & A limitar & 0 & $0 \%$ & 0 & $\mathrm{x}$ & Kappa & $\mathrm{x}$ & $\mathrm{x}$ & $\mathrm{x}$ & $\mathrm{x}$ \\
\hline & & & & & & & $\begin{array}{c}\text { Gwet's } \\
\text { AC1 }\end{array}$ & $\mathrm{x}$ & $\mathrm{x}$ & $\mathrm{x}$ & $\mathrm{x}$ \\
\hline \multirow{3}{*}{$\begin{array}{c}\text { Alimentos de } \\
\text { consumo } \\
\text { opcional }\end{array}$} & \multirow{3}{*}{932} & $\begin{array}{l}\text { A } \\
\text { promover }\end{array}$ & 0 & $\mathrm{x}$ & 57 & $6 \%$ & $\begin{array}{c}\% \\
\text { acuerdo }\end{array}$ & 94 & 91 & 96 & $\begin{array}{l}\text { Casi } \\
\text { Perfecto }\end{array}$ \\
\hline & & A limitar & 0 & $\mathrm{x}$ & $\begin{array}{c}87 \\
5\end{array}$ & $94 \%$ & Kappa & $\mathrm{x}$ & $\mathrm{x}$ & $\mathrm{x}$ & $\mathrm{x}$ \\
\hline & & & & & & & $\begin{array}{c}\text { Gwet's } \\
\text { AC1 }\end{array}$ & 0,936 & $\begin{array}{c}0,90 \\
8\end{array}$ & $\begin{array}{c}0,96 \\
3\end{array}$ & $\begin{array}{l}\text { Casi } \\
\text { Perfecto }\end{array}$ \\
\hline \multirow{3}{*}{$\begin{array}{l}\text { Bebidas sin } \\
\text { azúcar }\end{array}$} & \multirow{3}{*}{34} & $\begin{array}{l}\text { A } \\
\text { promover }\end{array}$ & 24 & $71 \%$ & 0 & $\mathrm{x}$ & $\begin{array}{c}\% \\
\text { acuerdo }\end{array}$ & 71 & 51 & 90 & Sustancial \\
\hline & & A limitar & 10 & $29 \%$ & 0 & $\mathrm{x}$ & Kappa & $\mathrm{x}$ & $\mathrm{x}$ & $\mathrm{x}$ & $\mathrm{x}$ \\
\hline & & & & & & & $\begin{array}{c}\text { Gwet's } \\
\text { AC1 }\end{array}$ & 0,628 & $\begin{array}{c}0,36 \\
6 \\
\end{array}$ & $\begin{array}{c}0,89 \\
0 \\
\end{array}$ & Sustancial \\
\hline \multirow{3}{*}{$\begin{array}{c}\text { Comidas pre- } \\
\text { elaboradas }\end{array}$} & \multirow{3}{*}{11} & $\begin{array}{l}\text { A } \\
\text { promover }\end{array}$ & 1 & $9 \%$ & 0 & $\mathrm{x}$ & $\begin{array}{c}\% \\
\text { acuerdo }\end{array}$ & 9 & $\mathrm{x}$ & $\mathrm{x}$ & $\begin{array}{l}\text { Insignifica } \\
\text { nte }\end{array}$ \\
\hline & & A limitar & 10 & $91 \%$ & 0 & $\mathrm{x}$ & Kappa & $\mathrm{x}$ & $\mathrm{x}$ & $\mathrm{x}$ & $\mathrm{x}$ \\
\hline & & & & & & & $\begin{array}{c}\text { Gwet's } \\
\text { AC1 }\end{array}$ & $\mathrm{x}$ & $\mathrm{x}$ & $\mathrm{x}$ & $\mathrm{x}$ \\
\hline $\begin{array}{l}\text { Muestra } \\
\text { Total** }\end{array}$ & $\begin{array}{c}155 \\
4\end{array}$ & & & & & & & & & & \\
\hline
\end{tabular}

* En la lectura de la tabla se reporta solo el Índice Gwet's AC1 por tener mayor robustez científica (Ver metodología)

** Los productos de la categoría sin dato se han descartado de la muestra total para el análisis del nivel de acuerdo (Ver metodología)

***No se ha podido realizar el cálculo por la naturaleza de los datos.

Fuente: Elaboración Propia

En cuanto al nivel de exigencia, las GAPA poseen un nivel de exigencia menor que el perfil de Uruguay $(71,2 \%$ vs $76,3 \%$ ) (Tabla 2). En la Tabla 8 se observa que a nivel de los grupos de alimentos, las GAPA son menos exigentes que el perfil de Uruguay en todos los grupos excepto en el grupo alimentos de consumo opcional (100\% GAPA vs $82,5 \%$ Uruguay), y en el grupo de aceite crudo, frutos secos y semillas donde ambos promueven la totalidad de los producto

En el grupo de legumbres, cereales, papa, pan y pastas el perfil de Uruguay coincide con las GAPA en limitar algunos productos de la categoría pastas rellenas (GAPA 100\% vs Uruguay $59,1 \%)$ por su alto contenido en sodio y grasas totales, y saturadas pero además limita productos de las siguientes categorías: $5,4 \%$ cereales refinados por su alto contenido de sodio, $50 \%$ pan blanco y $58,3 \%$ pan integral por su alto contenido en sodio y/o azúcares y $66,7 \%$ premezclas de panificados y cereales por su alto contenido en sodio, azúcares y/o grasas saturadas y $75 \%$ 
amasados por su alto contenido en sodio y grasas totales y/o grasas saturadas (Tabla 2 ANEXO III y Tabla 8).

En el grupo de frutas y verduras el perfil de Uruguay coincide con las GAPA en limitar todas las verduras en salmuera pero además clasifica a limitar productos dentro de las siguientes categorías por el alto contenido de sodio: $28 \%$ verduras y frutas enlatadas, y $66,7 \%$ comidas listas de verdura. (Tabla 2 ANEXO III y Tabla 8 )

En el grupo de Carnes y huevo el perfil de Uruguay coincide con las GAPA en limitar la mayoría de las carnes procesadas $(95,7 \%)$ pero además limita el $50 \%$ de las carnes enlatadas por el alto contenido de sodio, grasas saturadas y totales. (Tabla 2 ANEXO III y Tabla 7)

En el grupo de Leche yogur y quesos las GAPA promueven el consumo de todas las categorías y el perfil de Uruguay clasifica "a limitar" al 95\% de los quesos duros, semiduros y saborizados y al $91,2 \%$ de los quesos blandos por ser altos en sodio, grasas saturadas y totales (Tabla 2 ANEXO III y Tabla 8)

En el grupo de bebidas sin azúcar las GAPA y el perfil de Uruguay difieren en la clasificación de las bebidas edulcoradas, las GAPA promueven su consumo y el perfil clasifica "a limitar" a un 23,8\% de los productos por ser altos en sodio (presentan entre 9 y $21 \mathrm{mg}$ de sodio por caloría) (Tabla 2 ANEXO III y Tabla 8 ).

En comidas pre-elaboradas el perfil de Uruguay resulta más exigente limitando la mayoría de los productos (90,9\% vs GAPA $0 \%$ ) por su alto contenido en grasas saturadas, totales y sodio (Tabla 2 ANEXO III y Tabla 8)

Tabla 8: Nivel de exigencia del perfil de Uruguay las GAPA por grupos y por categorías de alimentos $(n=1703)$

\begin{tabular}{|c|c|c|c|c|c|c|c|c|}
\hline \multirow[t]{2}{*}{$\begin{array}{l}\text { Grupo de } \\
\text { alimento }\end{array}$} & \multirow[t]{2}{*}{$\begin{array}{c}\text { Categoría de } \\
\text { alimentos }\end{array}$} & \multirow[t]{2}{*}{$\mathrm{n}$} & & \multicolumn{2}{|c|}{$\begin{array}{c}\text { Clasificación } \\
\text { GAPA }\end{array}$} & \multicolumn{3}{|c|}{ Clasificación Perfil Uruguay } \\
\hline & & & & $\begin{array}{c}\text { A } \\
\text { Promover }\end{array}$ & $\begin{array}{c}\text { A } \\
\text { Limitar }\end{array}$ & $\begin{array}{c}\text { A } \\
\text { Promover }\end{array}$ & $\begin{array}{c}\text { A } \\
\text { Limitar }\end{array}$ & $\begin{array}{c}\text { Sin } \\
\text { dato* }^{*}\end{array}$ \\
\hline \multirow{13}{*}{$\begin{array}{l}\text { Legumbres, } \\
\text { cereales, papa, } \\
\text { pan y pastas }\end{array}$} & \multirow[t]{2}{*}{ Legumbres } & 2 & $\mathrm{n}$ & 2 & 0 & 2 & 0 & 0 \\
\hline & & & $\%$ & $100,0 \%$ & $0,0 \%$ & $100,0 \%$ & $0,0 \%$ & $0,0 \%$ \\
\hline & \multirow{2}{*}{$\begin{array}{l}\text { Legumbres } \\
\text { enlatadas }\end{array}$} & 7 & $\mathrm{n}$ & 7 & 0 & 7 & 0 & 0 \\
\hline & & & $\%$ & $100,0 \%$ & $0,0 \%$ & $100,0 \%$ & $0,0 \%$ & $0,0 \%$ \\
\hline & \multirow{2}{*}{$\begin{array}{l}\text { Cereales refinados: } \\
\text { arroz, fideos, pastas }\end{array}$} & 37 & $\mathrm{n}$ & 37 & 0 & 35 & 2 & 0 \\
\hline & & & $\%$ & $100,0 \%$ & $0,0 \%$ & $94,6 \%$ & $5,4 \%$ & $0,0 \%$ \\
\hline & \multirow[t]{2}{*}{ Cereales integrales } & 15 & $\mathrm{n}$ & 15 & 0 & 15 & 0 & 0 \\
\hline & & & $\%$ & $100,0 \%$ & $0,0 \%$ & $100,0 \%$ & $0,0 \%$ & $0,0 \%$ \\
\hline & \multirow[t]{2}{*}{ Pan blanco } & 48 & $\mathrm{n}$ & 48 & 0 & 24 & 24 & 0 \\
\hline & & & $\%$ & $100,0 \%$ & $0,0 \%$ & $50,0 \%$ & $50,0 \%$ & $0,0 \%$ \\
\hline & \multirow[t]{2}{*}{ Pan integral } & 12 & $\mathrm{n}$ & 12 & 0 & 4 & 7 & 1 \\
\hline & & & $\%$ & $100,0 \%$ & $0,0 \%$ & $33,3 \%$ & $58,3 \%$ & $8,3 \%$ \\
\hline & Hortalizas & 2 & $\mathrm{n}$ & 2 & 0 & 2 & 0 & 0 \\
\hline
\end{tabular}




\begin{tabular}{|c|c|c|c|c|c|c|c|c|}
\hline & feculentas & & $\%$ & $100,0 \%$ & $0,0 \%$ & $100,0 \%$ & $0,0 \%$ & $0,0 \%$ \\
\hline & \multirow[t]{2}{*}{ Pastas rellenas } & 22 & $\mathrm{n}$ & 0 & 22 & 8 & 13 & 1 \\
\hline & & & $\%$ & $0,0 \%$ & $100,0 \%$ & $36,4 \%$ & $59,1 \%$ & $4,5 \%$ \\
\hline & \multirow{2}{*}{$\begin{array}{l}\text { Premezclas } \\
\text { panificados y } \\
\text { cereales }\end{array}$} & 12 & $\mathrm{n}$ & 12 & 0 & 4 & 8 & 0 \\
\hline & & & $\%$ & $100,0 \%$ & $0,0 \%$ & $33,3 \%$ & $66,7 \%$ & $0,0 \%$ \\
\hline & \multirow[t]{2}{*}{ Amasados } & 28 & $\mathrm{n}$ & 28 & 0 & 6 & 21 & 1 \\
\hline & & & $\%$ & $100,0 \%$ & $0,0 \%$ & $21,4 \%$ & $75,0 \%$ & $3,6 \%$ \\
\hline \multirow{2}{*}{\multicolumn{2}{|c|}{ Total }} & 185 & $\mathrm{n}$ & 163 & 22 & 107 & 75 & 3 \\
\hline & & & $\%$ & $88,1 \%$ & $11,9 \%$ & $57,80 \%$ & $40,50 \%$ & $1,60 \%$ \\
\hline \multirow[t]{10}{*}{ Frutas y verduras } & \multirow[t]{2}{*}{ Frutas } & 2 & $\mathrm{n}$ & 2 & 0 & 2 & 0 & 0 \\
\hline & & & $\%$ & $100,0 \%$ & $0,0 \%$ & $100,0 \%$ & $0,0 \%$ & $0,0 \%$ \\
\hline & \multirow[t]{2}{*}{ Verduras } & 10 & $\mathrm{n}$ & 10 & 0 & 10 & 0 & 0 \\
\hline & & & $\%$ & $100,0 \%$ & $0,0 \%$ & $100,0 \%$ & $0,0 \%$ & $0,0 \%$ \\
\hline & \multirow{2}{*}{$\begin{array}{l}\text { Verduras y frutas } \\
\text { enlatadas }\end{array}$} & 25 & $\mathrm{n}$ & 25 & 0 & 18 & 7 & 0 \\
\hline & & & $\%$ & $100,0 \%$ & $0,0 \%$ & $72,0 \%$ & $28,0 \%$ & $0,0 \%$ \\
\hline & \multirow{2}{*}{$\begin{array}{l}\text { Verduras en } \\
\text { salmuera }\end{array}$} & 13 & $\mathrm{n}$ & 0 & 13 & 0 & 13 & 0 \\
\hline & & & $\%$ & $0,0 \%$ & $100,0 \%$ & $0,0 \%$ & $100,0 \%$ & $0,0 \%$ \\
\hline & \multirow{2}{*}{$\begin{array}{l}\text { Comidas listas de } \\
\text { verdura }\end{array}$} & 3 & $\mathrm{n}$ & 3 & 0 & 1 & 2 & 0 \\
\hline & & & $\%$ & $100,0 \%$ & $0,0 \%$ & $33,3 \%$ & $66,7 \%$ & $0,0 \%$ \\
\hline \multirow{2}{*}{\multicolumn{2}{|c|}{ Total }} & 53 & $\mathrm{n}$ & 40 & 13 & 31 & 22 & 0 \\
\hline & & & $\%$ & $75,5 \%$ & $24,5 \%$ & $58,50 \%$ & $41,50 \%$ & $0,00 \%$ \\
\hline \multirow[t]{8}{*}{ Carnes y huevo } & \multirow[t]{2}{*}{ Carnes } & 4 & $\mathrm{n}$ & 4 & 0 & 4 & 0 & 0 \\
\hline & & & $\%$ & $100,0 \%$ & $0,0 \%$ & $100,0 \%$ & $0,0 \%$ & $0,0 \%$ \\
\hline & \multirow[t]{2}{*}{ Huevo } & 1 & $\mathrm{n}$ & 1 & 0 & 1 & 0 & 0 \\
\hline & & & $\%$ & $100,0 \%$ & $0,0 \%$ & $100,0 \%$ & $0,0 \%$ & $0,0 \%$ \\
\hline & \multirow[t]{2}{*}{ Carnes enlatadas } & 16 & $\mathrm{n}$ & 16 & 0 & 7 & 8 & 1 \\
\hline & & & $\%$ & $100,0 \%$ & $0,0 \%$ & $43,8 \%$ & $50,0 \%$ & $6,3 \%$ \\
\hline & \multirow[t]{2}{*}{ Carnes procesadas } & 117 & $\mathrm{n}$ & 0 & 117 & 3 & 112 & 2 \\
\hline & & & $\%$ & $0,0 \%$ & $100,0 \%$ & $2,6 \%$ & $95,7 \%$ & $1,7 \%$ \\
\hline \multirow{2}{*}{\multicolumn{2}{|c|}{ Total }} & 138 & $\mathrm{n}$ & 21 & 117 & 15 & 120 & 3 \\
\hline & & & $\%$ & $15,2 \%$ & $84,8 \%$ & $10,90 \%$ & $87,00 \%$ & $2,20 \%$ \\
\hline \multirow{12}{*}{$\begin{array}{c}\text { Leche, yogur y } \\
\text { quesos }\end{array}$} & \multirow[t]{2}{*}{ Leche entera } & 2 & $\mathrm{n}$ & 2 & 0 & 2 & 0 & 0 \\
\hline & & & $\%$ & $100,0 \%$ & $0,0 \%$ & $100,0 \%$ & $0,0 \%$ & $0,0 \%$ \\
\hline & \multirow{2}{*}{$\begin{array}{l}\text { Leche parcialmente } \\
\text { descremada }\end{array}$} & 2 & $\mathrm{n}$ & 2 & 0 & 2 & 0 & 0 \\
\hline & & & $\%$ & $100,0 \%$ & $0,0 \%$ & $100,0 \%$ & $0,0 \%$ & $0,0 \%$ \\
\hline & \multirow[t]{2}{*}{ Yogur entero } & 7 & $\mathrm{n}$ & 7 & 0 & 0 & 2 & 5 \\
\hline & & & $\%$ & $100,0 \%$ & $0,0 \%$ & $0,0 \%$ & $28,6 \%$ & $71,4 \%$ \\
\hline & \multirow{2}{*}{$\begin{array}{l}\text { Yogur parcialmente } \\
\text { descremado }\end{array}$} & 4 & $\mathrm{n}$ & 4 & 0 & 4 & 0 & 0 \\
\hline & & & $\%$ & $100,0 \%$ & $0,0 \%$ & $100,0 \%$ & $0,0 \%$ & $0,0 \%$ \\
\hline & Queso duro, & 117 & $\mathrm{n}$ & 117 & 0 & 0 & 117 & 0 \\
\hline & $\begin{array}{l}\text { semiduro y } \\
\text { saborizados }\end{array}$ & & $\%$ & $100,0 \%$ & $0,0 \%$ & $0,0 \%$ & $100,0 \%$ & $0,0 \%$ \\
\hline & Quesos blandos & 73 & $\mathrm{n}$ & 73 & 0 & 4 & 68 & 1 \\
\hline & & & $\%$ & $100,0 \%$ & $0,0 \%$ & $5,5 \%$ & $93,2 \%$ & $1,4 \%$ \\
\hline Total & & 205 & $\mathrm{n}$ & 205 & 0 & 12 & 187 & 6 \\
\hline
\end{tabular}




\begin{tabular}{|c|c|c|c|c|c|c|c|c|}
\hline & & & $\%$ & $100,0 \%$ & $0,0 \%$ & $5,90 \%$ & $91,20 \%$ & $2,90 \%$ \\
\hline \multirow{6}{*}{$\begin{array}{l}\text { Aceite crudo, } \\
\text { frutos secos y } \\
\text { semillas }\end{array}$} & \multirow[t]{2}{*}{ Aceite } & 2 & $\mathrm{n}$ & 2 & 0 & 2 & 0 & 0 \\
\hline & & & $\%$ & $100,0 \%$ & $0,0 \%$ & $100,0 \%$ & $0,0 \%$ & $0,0 \%$ \\
\hline & \multirow[t]{2}{*}{ Frutos secos } & 4 & $\mathrm{n}$ & 4 & 0 & 4 & 0 & 0 \\
\hline & & & $\%$ & $100,0 \%$ & $0,0 \%$ & $100,0 \%$ & $0,0 \%$ & $0,0 \%$ \\
\hline & \multirow[t]{2}{*}{ Semillas } & 2 & $\mathrm{n}$ & 2 & 0 & 2 & 0 & 0 \\
\hline & & & $\%$ & $100,0 \%$ & $0,0 \%$ & $100,0 \%$ & $0,0 \%$ & $0,0 \%$ \\
\hline \multirow{2}{*}{\multicolumn{2}{|c|}{ Total }} & 8 & $\mathrm{n}$ & 8 & 0 & 8 & 0 & 0 \\
\hline & & & $\%$ & $100,0 \%$ & $0,0 \%$ & $100,00 \%$ & $0,00 \%$ & $0,00 \%$ \\
\hline \multirow{30}{*}{$\begin{array}{c}\text { Alimentos de } \\
\text { consumo opcional }\end{array}$} & \multirow[t]{2}{*}{ Alimentos grasos } & 38 & $\mathrm{n}$ & 0 & 38 & 0 & 37 & 1 \\
\hline & & & $\%$ & $0,0 \%$ & $100,0 \%$ & $0,0 \%$ & $97,4 \%$ & $2,6 \%$ \\
\hline & \multirow[t]{2}{*}{ Azúcares libres } & 3 & $\mathrm{n}$ & 0 & 3 & 1 & 2 & 0 \\
\hline & & & $\%$ & $0,0 \%$ & $100,0 \%$ & $33,3 \%$ & $66,7 \%$ & $0,0 \%$ \\
\hline & \multirow[t]{2}{*}{ Alimentos dulces } & 159 & $\mathrm{n}$ & 0 & 159 & 2 & 153 & 4 \\
\hline & & & $\%$ & $0,0 \%$ & $100,0 \%$ & $1,3 \%$ & $96,2 \%$ & $2,5 \%$ \\
\hline & \multirow[t]{2}{*}{ Galletitas } & 291 & $\mathrm{n}$ & 0 & 291 & 2 & 247 & 42 \\
\hline & & & $\%$ & $0,0 \%$ & $100,0 \%$ & $0,7 \%$ & $84,9 \%$ & $14,4 \%$ \\
\hline & \multirow[t]{2}{*}{ Postres azucarados } & 78 & $\mathrm{n}$ & 0 & 78 & 9 & 56 & 13 \\
\hline & & & $\%$ & $0,0 \%$ & $100,0 \%$ & $11,5 \%$ & $71,8 \%$ & $16,7 \%$ \\
\hline & \multirow[t]{2}{*}{ Bebidas azucaradas } & 101 & $\mathrm{n}$ & 0 & 101 & 39 & 62 & 0 \\
\hline & & & $\%$ & $0,0 \%$ & $100,0 \%$ & $38,6 \%$ & $61,4 \%$ & $0,0 \%$ \\
\hline & \multirow{2}{*}{$\begin{array}{l}\text { Panificados dulces o } \\
\text { salados }\end{array}$} & 51 & $\mathrm{n}$ & 0 & 51 & 0 & 35 & 16 \\
\hline & & & $\%$ & $0,0 \%$ & $100,0 \%$ & $0,0 \%$ & $68,6 \%$ & $31,4 \%$ \\
\hline & \multirow{2}{*}{$\begin{array}{l}\text { Premezclas } \\
\text { panificados dulces }\end{array}$} & 16 & $\mathrm{n}$ & 0 & 16 & 0 & 11 & 5 \\
\hline & & & $\%$ & $0,0 \%$ & $100,0 \%$ & $0,0 \%$ & $68,8 \%$ & $31,3 \%$ \\
\hline & \multirow{2}{*}{$\begin{array}{l}\text { Barras de cereal con } \\
\text { azúcar }\end{array}$} & 12 & $\mathrm{n}$ & 0 & 12 & 0 & 9 & 3 \\
\hline & & & $\%$ & $0,0 \%$ & $100,0 \%$ & $0,0 \%$ & $75,0 \%$ & $25,0 \%$ \\
\hline & \multirow{2}{*}{$\begin{array}{l}\text { Cereales de } \\
\text { desayuno con } \\
\text { azúcar }\end{array}$} & 63 & $\mathrm{n}$ & 0 & 63 & 0 & 42 & 21 \\
\hline & & & $\%$ & $0,0 \%$ & $100,0 \%$ & $0,0 \%$ & $66,7 \%$ & $33,3 \%$ \\
\hline & \multirow{2}{*}{$\begin{array}{l}\text { Snacks y productos } \\
\text { de copetin }\end{array}$} & 80 & $\mathrm{n}$ & 0 & 80 & 0 & 79 & 1 \\
\hline & & & $\%$ & $0,0 \%$ & $100,0 \%$ & $0,0 \%$ & $98,8 \%$ & $1,3 \%$ \\
\hline & \multirow[t]{2}{*}{ Comidas rápidas } & 55 & $\mathrm{n}$ & 0 & 55 & 3 & 41 & 11 \\
\hline & & & $\%$ & $0,0 \%$ & $100,0 \%$ & $5,5 \%$ & $74,5 \%$ & $20,0 \%$ \\
\hline & \multirow[t]{2}{*}{ Aderezos } & 57 & $\mathrm{n}$ & 0 & 57 & 0 & 51 & 6 \\
\hline & & & $\%$ & $0,0 \%$ & $100,0 \%$ & $0,0 \%$ & $89,5 \%$ & $10,5 \%$ \\
\hline & \multirow[t]{2}{*}{ Salsas comerciales } & 18 & $\mathrm{n}$ & 0 & 18 & 0 & 14 & 4 \\
\hline & & & $\%$ & $0,0 \%$ & $100,0 \%$ & $0,0 \%$ & $77,8 \%$ & $22,2 \%$ \\
\hline & \multirow{2}{*}{$\begin{array}{l}\text { Caldos, } \\
\text { saborizadores y } \\
\text { sopas comerciales }\end{array}$} & 39 & $\mathrm{n}$ & 0 & 39 & 1 & 36 & 2 \\
\hline & & & $\%$ & $0,0 \%$ & $100,0 \%$ & $2,6 \%$ & $92,3 \%$ & $5,1 \%$ \\
\hline \multirow{2}{*}{ Total } & & 1061 & $\mathrm{n}$ & 0 & 1061 & 57 & 875 & 129 \\
\hline & & & $\%$ & $0,0 \%$ & $100,0 \%$ & $5,40 \%$ & $82,50 \%$ & $12,20 \%$ \\
\hline \multirow{3}{*}{$\begin{array}{l}\text { Bebidas sin } \\
\text { azúcar }\end{array}$} & Soda & 1 & $\mathrm{n}$ & 1 & 0 & 1 & 0 & 0 \\
\hline & & & $\%$ & $100,0 \%$ & $0,0 \%$ & $100,0 \%$ & $0,0 \%$ & $0,0 \%$ \\
\hline & Aguas minerales & 7 & $\mathrm{n}$ & 7 & 0 & 7 & 0 & 0 \\
\hline
\end{tabular}




\begin{tabular}{|l|l|l|l|r|r|r|r|r|} 
& & $\%$ & $100,0 \%$ & $0,0 \%$ & $100,0 \%$ & $0,0 \%$ & $0,0 \%$ \\
\cline { 2 - 8 } & Infusiones & 1 & $\mathrm{n}$ & 1 & 0 & 1 & 0 & 0 \\
\cline { 2 - 8 } & & $\%$ & $100,0 \%$ & $0,0 \%$ & $100,0 \%$ & $0,0 \%$ & $0,0 \%$ \\
\cline { 2 - 8 } $\begin{array}{l}\text { Bebidas } \\
\text { edulcoradas }\end{array}$ & 33 & $\mathrm{n}$ & 33 & 0 & 15 & 10 & 8 \\
\cline { 2 - 8 } & & $\%$ & $100,0 \%$ & $0,0 \%$ & $45,5 \%$ & $30,3 \%$ & $24,2 \%$ \\
\hline \multirow{3}{*}{ Total } & 42 & $\mathrm{n}$ & 42 & 0 & 24 & 10 & 8 \\
\cline { 2 - 8 } & & $\%$ & $100,0 \%$ & $0,0 \%$ & $57,10 \%$ & $23,80 \%$ & $19,00 \%$ \\
\hline Comidas pre-elaboradas & 11 & $\mathrm{n}$ & 11 & 0 & 1 & 10 & 0 \\
\cline { 2 - 8 } & & $\%$ & $100,0 \%$ & $0,0 \%$ & $9,1 \%$ & $90,9 \%$ & $0,0 \%$ \\
\hline Muestra Total & 1703 & $\mathrm{n}$ & 490 & 1213 & 255 & 1299 & 149 \\
\hline
\end{tabular}

* Alimentos que no pudieron clasificarse por falta de información.

Fuente: Elaboración Propia.

\section{Perfil Chile y GAPA}

El Perfil de Chile presenta un acuerdo moderado con las GAPA (Gwet's AC1=0,558) (Tabla 4). La Tabla 9 muestra que alcanza el mayor nivel de acuerdo en los grupos de alimentos de carnes y huevos (Gwet's $\mathrm{AC} 1=0,821)$, alimentos de consumo opcional (Gwet's $\mathrm{AC} 1=0,869)$, y frutas y verduras (Gwet's $\mathrm{AC} 1=0,810$ ) con un acuerdo casi perfecto.En los grupos de legumbres, cereales, papa, pan y pastas (Gwet's $A C 1=-0,455)$ y leche, yogures y quesos no se encontró acuerdo (Gwet's $\mathrm{AC} 1=-0,863$ ). En los grupos donde no se pudo calcular el Índice Gwet's AC1 ni el Kappa, el porcentaje de acuerdo muestra un acuerdo moderado en el grupo comidas pre-elaboradas y un acuerdo perfecto en el grupo aceite crudo, frutos secos y semillas y en bebidas sin azúcar.

Tabla 9: Nivel de acuerdo entre el perfil de Chile y las GAPA por grupos de alimentos $(\mathrm{n}=1634)$

\begin{tabular}{|c|c|c|c|c|c|c|c|c|c|c|c|}
\hline \multirow{3}{*}{$\begin{array}{l}\text { Grupo de } \\
\text { alimento }\end{array}$} & \multirow{3}{*}{$\mathrm{n}$} & \multirow{3}{*}{$\begin{array}{c}\text { Clasificaci } \\
\text { on Perfil } \\
\text { Chile }\end{array}$} & \multicolumn{4}{|c|}{ Clasificación GAPA } & \multicolumn{5}{|c|}{ Nivel de acuerdo* } \\
\hline & & & \multicolumn{2}{|c|}{$\begin{array}{c}\mathrm{A} \\
\text { promover }\end{array}$} & \multicolumn{2}{|c|}{$\begin{array}{c}\text { A } \\
\text { limitar }\end{array}$} & \multirow{2}{*}{ Indicador } & \multirow{2}{*}{$\begin{array}{l}\text { Estimaci } \\
\text { ón }\end{array}$} & \multirow{2}{*}{\multicolumn{2}{|c|}{ IC $95 \%$}} & \multirow{2}{*}{$\begin{array}{l}\text { Interpretaci } \\
\text { ón } \\
\text { probabilísti } \\
\text { ca }\end{array}$} \\
\hline & & & $\mathrm{N}$ & $\%$ & $\mathrm{~N}$ & $\%$ & & & & & \\
\hline \multirow{3}{*}{$\begin{array}{c}\text { Legumbres, } \\
\text { cereales, } \\
\text { papa, pan y } \\
\text { pastas }\end{array}$} & \multirow{3}{*}{183} & \begin{tabular}{|l} 
A \\
promover
\end{tabular} & 31 & $19 \%$ & 4 & $\begin{array}{l}18 \\
\% \\
\end{array}$ & $\begin{array}{c}\% \\
\text { acuerdo }\end{array}$ & 27 & 21 & 33 & Discreto \\
\hline & & A limitar & $\begin{array}{c}13 \\
0\end{array}$ & $81 \%$ & 18 & $\begin{array}{l}82 \\
\%\end{array}$ & Kappa & 0,004 & $\begin{array}{c}- \\
0,04 \\
6 \\
\end{array}$ & $\begin{array}{c}0,05 \\
4\end{array}$ & $\begin{array}{l}\text { Insignifica } \\
\text { nte }\end{array}$ \\
\hline & & & & & & & $\begin{array}{c}\text { Gwet's } \\
\text { AC1 }\end{array}$ & $-0,455$ & $\begin{array}{c}- \\
0,58 \\
9\end{array}$ & $\begin{array}{c}- \\
0,32 \\
1 \\
\end{array}$ & $\begin{array}{l}\text { Sin } \\
\text { Acuerdo }\end{array}$ \\
\hline \multirow{3}{*}{$\begin{array}{l}\text { Frutas y } \\
\text { verduras }\end{array}$} & \multirow{3}{*}{53} & $\begin{array}{l}\text { A } \\
\text { promover }\end{array}$ & 35 & $88 \%$ & 1 & $8 \%$ & $\begin{array}{c}\% \\
\text { acuerdo }\end{array}$ & 89 & 80 & 98 & $\begin{array}{l}\text { Casi } \\
\text { Perfecto }\end{array}$ \\
\hline & & A limitar & 5 & $13 \%$ & 12 & $\begin{array}{l}92 \\
\% \\
\end{array}$ & Kappa & 0,723 & $\begin{array}{c}0,51 \\
2\end{array}$ & $\begin{array}{c}0,93 \\
4\end{array}$ & Sustancial \\
\hline & & & & & & & $\begin{array}{c}\text { Gwet's } \\
\text { AC1 }\end{array}$ & 0,810 & $\begin{array}{c}0,65 \\
2 \\
\end{array}$ & $\begin{array}{c}0,96 \\
7 \\
\end{array}$ & $\begin{array}{l}\text { Casi } \\
\text { Perfecto }\end{array}$ \\
\hline $\begin{array}{c}\text { Carnes y } \\
\text { huevo }\end{array}$ & 136 & $\begin{array}{l}\text { A } \\
\text { promover }\end{array}$ & 15 & $79 \%$ & 13 & $\begin{array}{l}11 \\
\%\end{array}$ & $\begin{array}{c}\% \\
\text { acuerdo }\end{array}$ & 88 & 82 & 93 & $\begin{array}{l}\text { Casi } \\
\text { Perfecto }\end{array}$ \\
\hline
\end{tabular}




\begin{tabular}{|c|c|c|c|c|c|c|c|c|c|c|c|}
\hline & & A limitar & 4 & $21 \%$ & $\begin{array}{c}10 \\
4\end{array}$ & $\begin{array}{l}89 \\
\%\end{array}$ & Kappa & 0,579 & $\begin{array}{c}0,39 \\
9 \\
\end{array}$ & $\begin{array}{c}0,75 \\
5\end{array}$ & Moderado \\
\hline & & & & & & & $\begin{array}{c}\text { Gwet's } \\
\text { AC1 }\end{array}$ & 0,821 & $\begin{array}{c}0,73 \\
0\end{array}$ & $\begin{array}{c}0,91 \\
3\end{array}$ & $\begin{array}{l}\text { Casi } \\
\text { Perfecto }\end{array}$ \\
\hline \multirow{3}{*}{$\begin{array}{l}\text { Leche, yogur } \\
\text { y quesos }\end{array}$} & \multirow{3}{*}{198} & $\begin{array}{l}\text { A } \\
\text { promover }\end{array}$ & 12 & $6 \%$ & 0 & $\begin{array}{c}\mathrm{X}^{* *} \\
*\end{array}$ & $\begin{array}{c}\% \\
\text { acuerdo }\end{array}$ & 6 & 3 & 9 & $\begin{array}{l}\text { Insignifica } \\
\text { nte }\end{array}$ \\
\hline & & A limitar & $\begin{array}{c}18 \\
6 \\
\end{array}$ & $94 \%$ & 0 & $\mathrm{x}$ & Kappa & 0 & 0 & 0 & $\begin{array}{l}\text { Sin } \\
\text { Acuerdo }\end{array}$ \\
\hline & & & & & & & $\begin{array}{l}\text { Gwet's } \\
\text { AC1 }\end{array}$ & $-0,863$ & $\begin{array}{c}- \\
0,94 \\
6\end{array}$ & $\begin{array}{c}- \\
0,78 \\
0\end{array}$ & $\begin{array}{l}\text { Sin } \\
\text { Acuerdo }\end{array}$ \\
\hline \multirow{3}{*}{$\begin{array}{l}\text { Aceite crudo, } \\
\text { frutos secos y } \\
\text { semillas }\end{array}$} & \multirow{3}{*}{8} & $\begin{array}{l}\text { A } \\
\text { promover }\end{array}$ & 8 & $\begin{array}{c}100 \\
\% \\
\end{array}$ & 0 & $\mathrm{x}$ & $\begin{array}{c}\% \% \\
\text { acuerdo }\end{array}$ & 100 & $\mathrm{x}$ & $\mathrm{x}$ & Perfecto \\
\hline & & A limitar & 0 & $0 \%$ & 0 & $\mathrm{x}$ & Kappa & $\mathrm{x}$ & $\mathrm{x}$ & $\mathrm{x}$ & $\mathrm{x}$ \\
\hline & & & & & & & $\begin{array}{c}\text { Gwet's } \\
\text { AC1 }\end{array}$ & $\mathrm{x}$ & $\mathrm{x}$ & $\mathrm{x}$ & $\mathrm{x}$ \\
\hline \multirow{3}{*}{$\begin{array}{l}\text { Alimentos de } \\
\text { consumo } \\
\text { opcional }\end{array}$} & \multirow{3}{*}{$\begin{array}{c}100 \\
4\end{array}$} & $\begin{array}{l}\text { A } \\
\text { promover }\end{array}$ & 0 & $\mathrm{x}$ & $\begin{array}{c}11 \\
8\end{array}$ & $\begin{array}{l}12 \\
\%\end{array}$ & $\begin{array}{c}\% \\
\text { acuerdo }\end{array}$ & 88 & 86 & 91 & $\begin{array}{l}\text { Casi } \\
\text { Perfecto }\end{array}$ \\
\hline & & A limitar & 0 & $\mathrm{x}$ & $\begin{array}{c}88 \\
6 \\
\end{array}$ & $\begin{array}{l}88 \\
\%\end{array}$ & Карра & $\mathrm{x}$ & $\mathrm{x}$ & $\mathrm{x}$ & $\mathrm{x}$ \\
\hline & & & & & & & $\begin{array}{c}\text { Gwet's } \\
\text { AC1 }\end{array}$ & 0,869 & $\begin{array}{c}0,84 \\
1 \\
\end{array}$ & $\begin{array}{c}0,89 \\
6 \\
\end{array}$ & $\begin{array}{l}\text { Casi } \\
\text { Perfecto }\end{array}$ \\
\hline \multirow{3}{*}{$\begin{array}{l}\text { Bebidas sin } \\
\text { azúcar }\end{array}$} & \multirow{3}{*}{41} & $\begin{array}{l}\text { A } \\
\text { promover }\end{array}$ & 41 & $\begin{array}{c}100 \\
\%\end{array}$ & 0 & $\mathrm{x}$ & $\begin{array}{c}\% \\
\text { acuerdo }\end{array}$ & 100 & $\mathrm{x}$ & $\mathrm{x}$ & Perfecto \\
\hline & & A limitar & 0 & $0 \%$ & 0 & $\mathrm{x}$ & Kappa & $\mathrm{x}$ & $\mathrm{x}$ & $\mathrm{x}$ & $\mathrm{x}$ \\
\hline & & & & & & & $\begin{array}{c}\text { Gwet's } \\
\text { ACl }\end{array}$ & $\mathrm{x}$ & $\mathrm{x}$ & $\mathrm{x}$ & $\mathrm{x}$ \\
\hline \multirow{3}{*}{$\begin{array}{c}\text { Comidas pre- } \\
\text { elaboradas }\end{array}$} & \multirow{3}{*}{11} & $\begin{array}{l}\text { A } \\
\text { promover }\end{array}$ & 6 & $55 \%$ & 0 & $\mathrm{x}$ & $\begin{array}{c}\% \\
\text { acuerdo }\end{array}$ & 55 & 20 & 90 & Moderado \\
\hline & & A limitar & 5 & $45 \%$ & 0 & $\mathrm{x}$ & Kappa & $\mathrm{x}$ & $\mathrm{x}$ & $\mathrm{x}$ & $\mathrm{x}$ \\
\hline & & & & & & & $\begin{array}{c}\text { Gwet's } \\
\text { AC1 }\end{array}$ & $\mathrm{x}$ & $\mathrm{x}$ & $\mathrm{x}$ & $\mathrm{x}$ \\
\hline $\begin{array}{l}\text { Muestra } \\
\text { Total** }\end{array}$ & $\begin{array}{c}163 \\
4 \\
\end{array}$ & & & & & & & & & & \\
\hline
\end{tabular}

* En la lectura de la tabla se reporta solo el Índice Gwet's AC1 por tener mayor robustez científica (Ver metodología)

** Los productos de la categoría sin dato se han descartado de la muestra total para el análisis del nivel de acuerdo (Ver metodología)

*** No se ha podido realizar el cálculo por la naturaleza de los datos.

Fuente: Elaboración Propia

En cuanto al nivel de exigencia, las GAPA poseen un nivel de exigencia menor que el perfil de Chile (71,2\%vs 79,3\%) (Tabla 2). En la Tabla 9 se observa que a nivel de los grupos de alimentos, las GAPA son menos exigentes que el perfil de Chile en todos los grupos excepto en el grupo alimentos de consumo opcional (100\% GAPA vs $82,5 \%$ Chile), y en el grupo de aceite crudo, frutos secos y semillas y bebidas sin azúcar (0\% GAPA y 0\% OPS).

En el grupo de legumbres, cereales, papa, pan y pastas, el perfil de Chile es más exigente que las GAPA (Nivel de exigencia: GAPA 11,9 vs perfil de Chile 80\%). Coincide con las GAPA en limitar la mayoría de las pastas rellenas $(81,8 \%)$ por su alto contenido en sodio y 
grasas totales, y energía pero además limita productos de las siguientes categorías: 100\% legumbres enlatadas y $97,3 \%$ cereales refinados por su alto contenido de sodio y/o energía, $95,8 \%$ pan blanco y $83,3 \%$ pan integral por su alto contenido en sodio, azúcares y/o energía, el $100 \%$ premezclas de panificados y cereales por su alto contenido en sodio, grasas saturadas, azúcares y energía, y el $89,3 \%$ amasados por su alto contenido en sodio, grasas saturadas, y/o energía. (Tabla 3 ANEXO III y Tabla 9).

En el grupo de frutas y verduras el perfil de Chile coincide con las GAPA en limitar las verduras en salmuera $(92,3 \%)$ pero además limita productos de las siguientes categorías: $12 \%$ de verduras y frutas enlatadas por el alto contenido de sodio y $66,7 \%$ de las comidas listas de verdura por el alto contenido en sodio y/o energía. (Tabla 3 ANEXO III y Tabla 9).

En el grupo de leche, yogur y quesos, difieren en la clasificación de las categorías de quesos, las GAPA promueven su consumo mientras que el perfil de Chile clasifica "a limitar" a un $100 \%$ de los quesos duros, semiduros y saborizados y un $94,5 \%$ de los quesos blandos, ambos por su alto contenido en sodio, grasas saturadas, y/o energía (Tabla 3 ANEXO III y Tabla 9)

En las comidas pre-elaboradas el perfil de Chile difiere con las GAPA en la clasificación del $45,5 \%$ de los productos incluidos, clasificándolos "a limitar" por su alto contenido en sodio y/o grasas saturadas (Tabla 3 ANEXO III y Tabla 9)

Tabla 9: Nivel de exigencia del perfil de Chile y las GAPA por grupos y por categorías de alimentos ( $\mathrm{n}=1703)$

\begin{tabular}{|c|c|c|c|c|c|c|c|c|}
\hline \multirow[t]{2}{*}{$\begin{array}{l}\text { Grupo de } \\
\text { alimento }\end{array}$} & \multirow[t]{2}{*}{$\begin{array}{l}\text { Categoría de } \\
\text { alimentos }\end{array}$} & \multirow[t]{2}{*}{$\mathrm{n}$} & & \multicolumn{2}{|c|}{$\begin{array}{l}\text { Clasificación } \\
\text { GAPA }\end{array}$} & \multicolumn{3}{|c|}{ Clasificación Perfil Chile } \\
\hline & & & & $\begin{array}{c}\text { A } \\
\text { Promover }\end{array}$ & $\begin{array}{c}\text { A } \\
\text { Limitar }\end{array}$ & $\begin{array}{c}\text { A } \\
\text { Promover }\end{array}$ & $\begin{array}{c}\mathrm{A} \\
\text { Limitar }\end{array}$ & $\begin{array}{c}\text { Sin } \\
\text { dato*** }\end{array}$ \\
\hline \multirow{15}{*}{$\begin{array}{l}\text { Legumbres, } \\
\text { cereales, papa, } \\
\text { pan y pastas }\end{array}$} & \multirow[t]{2}{*}{ Legumbres } & \multirow[t]{2}{*}{2} & $\mathrm{n}$ & 2 & 0 & 2 & 0 & 0 \\
\hline & & & $\%$ & $100,0 \%$ & $0,0 \%$ & $100,0 \%$ & $0,0 \%$ & $0,0 \%$ \\
\hline & \multirow{2}{*}{$\begin{array}{l}\text { Legumbres } \\
\text { enlatadas }\end{array}$} & \multirow[t]{2}{*}{7} & $\mathrm{n}$ & 7 & 0 & 7 & 0 & 0 \\
\hline & & & $\%$ & $100,0 \%$ & $0,0 \%$ & $100,0 \%$ & $0,0 \%$ & $0,0 \%$ \\
\hline & \multirow{2}{*}{$\begin{array}{l}\text { Cereales refinados: } \\
\text { arroz, fideos, pastas }\end{array}$} & \multirow[t]{2}{*}{37} & $\mathrm{n}$ & 37 & 0 & 1 & 36 & 0 \\
\hline & & & $\%$ & $100,0 \%$ & $0,0 \%$ & $2,7 \%$ & $97,3 \%$ & $0,0 \%$ \\
\hline & \multirow[t]{2}{*}{ Cereales integrales } & \multirow[t]{2}{*}{15} & $\mathrm{n}$ & 15 & 0 & 14 & 1 & 0 \\
\hline & & & $\%$ & $100,0 \%$ & $0,0 \%$ & $93,3 \%$ & $6,7 \%$ & $0,0 \%$ \\
\hline & \multirow[t]{2}{*}{ Pan blanco } & \multirow[t]{2}{*}{48} & $\mathrm{n}$ & 48 & 0 & 2 & 46 & 0 \\
\hline & & & $\%$ & $100,0 \%$ & $0,0 \%$ & $4,2 \%$ & $95,8 \%$ & $0,0 \%$ \\
\hline & \multirow[t]{2}{*}{ Pan integral } & \multirow[t]{2}{*}{12} & $\mathrm{n}$ & 12 & 0 & 1 & 10 & 1 \\
\hline & & & $\%$ & $100,0 \%$ & $0,0 \%$ & $8,3 \%$ & $83,3 \%$ & $8,3 \%$ \\
\hline & \multirow{2}{*}{$\begin{array}{l}\text { Hortalizas } \\
\text { feculentas }\end{array}$} & \multirow[t]{2}{*}{2} & $\mathrm{n}$ & 2 & 0 & 2 & 0 & 0 \\
\hline & & & $\%$ & $100,0 \%$ & $0,0 \%$ & $100,0 \%$ & $0,0 \%$ & $0,0 \%$ \\
\hline & Pastas rellenas & 22 & $\mathrm{n}$ & 0 & 22 & 4 & 18 & 0 \\
\hline
\end{tabular}




\begin{tabular}{|c|c|c|c|c|c|c|c|c|}
\hline & & & $\%$ & $0,0 \%$ & $100,0 \%$ & $18,2 \%$ & $81,8 \%$ & $0,0 \%$ \\
\hline & \multirow{2}{*}{$\begin{array}{l}\text { Premezclas } \\
\text { panificados y } \\
\text { cereales }\end{array}$} & \multirow[t]{2}{*}{12} & $\mathrm{n}$ & 12 & 0 & 0 & 12 & 0 \\
\hline & & & $\%$ & $100,0 \%$ & $0,0 \%$ & $0,0 \%$ & $100,0 \%$ & $0,0 \%$ \\
\hline & \multirow[t]{2}{*}{ Amasados } & \multirow[t]{2}{*}{28} & $\mathrm{n}$ & 28 & 0 & 2 & 25 & 1 \\
\hline & & & $\%$ & $100,0 \%$ & $0,0 \%$ & $7,1 \%$ & $89,3 \%$ & $3,6 \%$ \\
\hline \multirow{2}{*}{\multicolumn{2}{|c|}{ Total }} & \multirow[t]{2}{*}{185} & $\mathrm{n}$ & 163 & 22 & 35 & 148 & 2 \\
\hline & & & $\%$ & $88,1 \%$ & $11,9 \%$ & $18,9 \%$ & $80,0 \%$ & $1,1 \%$ \\
\hline \multirow[t]{10}{*}{ Frutas y verduras } & \multirow[t]{2}{*}{ Frutas } & \multirow[t]{2}{*}{2} & $\mathrm{n}$ & 2 & 0 & 2 & 0 & 0 \\
\hline & & & $\%$ & $100,0 \%$ & $0,0 \%$ & $100,0 \%$ & $0,0 \%$ & $0,0 \%$ \\
\hline & \multirow[t]{2}{*}{ Verduras } & \multirow[t]{2}{*}{10} & $\mathrm{n}$ & 10 & 0 & 10 & 0 & 0 \\
\hline & & & $\%$ & $100,0 \%$ & $0,0 \%$ & $100,0 \%$ & $0,0 \%$ & $0,0 \%$ \\
\hline & \multirow{2}{*}{$\begin{array}{l}\text { Verduras y frutas } \\
\text { enlatadas }\end{array}$} & \multirow[t]{2}{*}{25} & $\mathrm{n}$ & 25 & 0 & 22 & 3 & 0 \\
\hline & & & $\%$ & $100,0 \%$ & $0,0 \%$ & $88,0 \%$ & $12,0 \%$ & $0,0 \%$ \\
\hline & \multirow{2}{*}{$\begin{array}{l}\text { Verduras en } \\
\text { salmuera }\end{array}$} & \multirow[t]{2}{*}{13} & $\mathrm{n}$ & 0 & 13 & 1 & 12 & 0 \\
\hline & & & $\%$ & $0,0 \%$ & $100,0 \%$ & $7,7 \%$ & $92,3 \%$ & $0,0 \%$ \\
\hline & \multirow{2}{*}{$\begin{array}{l}\text { Comidas listas de } \\
\text { verdura }\end{array}$} & \multirow[t]{2}{*}{3} & $\mathrm{n}$ & 3 & 0 & 1 & 2 & 0 \\
\hline & & & $\%$ & $100,0 \%$ & $0,0 \%$ & $33,3 \%$ & $66,7 \%$ & $0,0 \%$ \\
\hline \multirow{2}{*}{\multicolumn{2}{|c|}{ Total }} & \multirow[t]{2}{*}{53} & $\mathrm{n}$ & 40 & 13 & 36 & 17 & 0 \\
\hline & & & $\%$ & $75,5 \%$ & $24,5 \%$ & $67,9 \%$ & $32,1 \%$ & $0,0 \%$ \\
\hline \multirow[t]{8}{*}{ Carnes y huevo } & \multirow[t]{2}{*}{ Carnes } & \multirow[t]{2}{*}{4} & $\mathrm{n}$ & 4 & 0 & 4 & 0 & 0 \\
\hline & & & $\%$ & $100,0 \%$ & $0,0 \%$ & $100,0 \%$ & $0,0 \%$ & $0,0 \%$ \\
\hline & Huevo & 1 & $\mathrm{n}$ & 1 & 0 & 1 & 0 & 0 \\
\hline & & & $\%$ & $100,0 \%$ & $0,0 \%$ & $100,0 \%$ & $0,0 \%$ & $0,0 \%$ \\
\hline & Carnes enlatadas & 16 & $\mathrm{n}$ & 16 & 0 & 10 & 4 & 2 \\
\hline & & & $\%$ & $100,0 \%$ & $0,0 \%$ & $62,5 \%$ & $25,0 \%$ & $12,5 \%$ \\
\hline & Carnes procesadas & 117 & $\mathrm{n}$ & 0 & 117 & 13 & 104 & 0 \\
\hline & & & $\%$ & $0,0 \%$ & $100,0 \%$ & $11,1 \%$ & $88,9 \%$ & $0,0 \%$ \\
\hline & & 138 & $\mathrm{n}$ & 21 & 117 & 28 & 108 & 2 \\
\hline lotal & & & $\%$ & $15,2 \%$ & $84,8 \%$ & $20,3 \%$ & $78,3 \%$ & $1,4 \%$ \\
\hline Leche, yogur y & Leche entera & 2 & $\mathrm{n}$ & 2 & 0 & 2 & 0 & 0 \\
\hline quesos & & & $\%$ & $100,0 \%$ & $0,0 \%$ & $100,0 \%$ & $0,0 \%$ & $0,0 \%$ \\
\hline & Leche parcialmente & 2 & $\mathrm{n}$ & 2 & 0 & 2 & 0 & 0 \\
\hline & descremada & & $\%$ & $100,0 \%$ & $0,0 \%$ & $100,0 \%$ & $0,0 \%$ & $0,0 \%$ \\
\hline & Yogur entero & 7 & $\mathrm{n}$ & 7 & 0 & 0 & 0 & 7 \\
\hline & & & $\%$ & $100,0 \%$ & $0,0 \%$ & $0,0 \%$ & $0,0 \%$ & $100,0 \%$ \\
\hline & \begin{tabular}{|l|} 
Yogur parcialmente \\
\end{tabular} & 4 & $\mathrm{n}$ & 4 & 0 & 4 & 0 & 0 \\
\hline & descremado & & $\%$ & $100,0 \%$ & $0,0 \%$ & $100,0 \%$ & $0,0 \%$ & $0,0 \%$ \\
\hline & Queso duro, & 117 & $\mathrm{n}$ & 117 & 0 & 0 & 117 & 0 \\
\hline & $\begin{array}{l}\text { semiduro y } \\
\text { saborizados }\end{array}$ & & $\%$ & $100,0 \%$ & $0,0 \%$ & $0,0 \%$ & $100,0 \%$ & $0,0 \%$ \\
\hline & Quesos blandos & 73 & $\mathrm{n}$ & 73 & 0 & 4 & 69 & 0 \\
\hline & & & $\%$ & $100,0 \%$ & $0,0 \%$ & $5,5 \%$ & $94,5 \%$ & $0,0 \%$ \\
\hline & & 205 & $\mathrm{n}$ & 205 & 0 & 12 & 186 & 7 \\
\hline Total & & & $\%$ & $100,0 \%$ & $0,0 \%$ & $5,9 \%$ & $90,7 \%$ & $3,4 \%$ \\
\hline Aceite crudo, & Aceite & 2 & $\mathrm{n}$ & 2 & 0 & 2 & 0 & 0 \\
\hline
\end{tabular}




\begin{tabular}{|c|c|c|c|c|c|c|c|c|}
\hline \multirow{5}{*}{$\begin{array}{l}\text { frutos secos y } \\
\text { semillas }\end{array}$} & & & $\%$ & $100,0 \%$ & $0,0 \%$ & $100,0 \%$ & $0,0 \%$ & $0,0 \%$ \\
\hline & \multirow[t]{2}{*}{ Frutos secos } & \multirow[t]{2}{*}{4} & $\mathrm{n}$ & 4 & 0 & 4 & 0 & 0 \\
\hline & & & $\%$ & $100,0 \%$ & $0,0 \%$ & $100,0 \%$ & $0,0 \%$ & $0,0 \%$ \\
\hline & \multirow[t]{2}{*}{ Semillas } & \multirow[t]{2}{*}{2} & $\mathrm{n}$ & 2 & 0 & 2 & 0 & 0 \\
\hline & & & $\%$ & $100,0 \%$ & $0,0 \%$ & $100,0 \%$ & $0,0 \%$ & $0,0 \%$ \\
\hline \multirow{2}{*}{\multicolumn{2}{|c|}{ Total }} & \multirow[t]{2}{*}{8} & $\mathrm{n}$ & 8 & 0 & 8 & 0 & 0 \\
\hline & & & $\%$ & $100,0 \%$ & $0,0 \%$ & $100,0 \%$ & $0,0 \%$ & $0,0 \%$ \\
\hline \multirow{30}{*}{$\begin{array}{c}\text { Alimentos de } \\
\text { consumo opcional }\end{array}$} & \multirow[t]{2}{*}{ Alimentos grasos } & \multirow[t]{2}{*}{38} & $\mathrm{n}$ & 0 & 38 & 0 & 38 & 0 \\
\hline & & & $\%$ & $0,0 \%$ & $100,0 \%$ & $0,0 \%$ & $100,0 \%$ & $0,0 \%$ \\
\hline & \multirow[t]{2}{*}{ Azúcares libres } & \multirow[t]{2}{*}{3} & $\mathrm{n}$ & 0 & 3 & 0 & 3 & 0 \\
\hline & & & $\%$ & $0,0 \%$ & $100,0 \%$ & $0,0 \%$ & $100,0 \%$ & $0,0 \%$ \\
\hline & \multirow[t]{2}{*}{ Alimentos dulces } & \multirow[t]{2}{*}{159} & $\mathrm{n}$ & 0 & 159 & 5 & 151 & 3 \\
\hline & & & $\%$ & $0,0 \%$ & $100,0 \%$ & $3,1 \%$ & $95,0 \%$ & $1,9 \%$ \\
\hline & \multirow[t]{2}{*}{ Galletitas } & \multirow[t]{2}{*}{291} & $\mathrm{n}$ & 0 & 291 & 0 & 290 & 1 \\
\hline & & & $\%$ & $0,0 \%$ & $100,0 \%$ & $0,0 \%$ & $99,7 \%$ & $0,3 \%$ \\
\hline & \multirow[t]{2}{*}{ Postres azucarados } & \multirow[t]{2}{*}{78} & $\mathrm{n}$ & 0 & 78 & 30 & 7 & 41 \\
\hline & & & $\%$ & $0,0 \%$ & $100,0 \%$ & $38,5 \%$ & $9,0 \%$ & $52,6 \%$ \\
\hline & \multirow[t]{2}{*}{ Bebidas azucaradas } & \multirow[t]{2}{*}{101} & $\mathrm{n}$ & 0 & 101 & 42 & 59 & 0 \\
\hline & & & $\%$ & $0,0 \%$ & $100,0 \%$ & $41,6 \%$ & $58,4 \%$ & $0,0 \%$ \\
\hline & \multirow{2}{*}{$\begin{array}{l}\text { Panificados dulces } \\
\text { o salados }\end{array}$} & \multirow[t]{2}{*}{51} & $\mathrm{n}$ & 0 & 51 & 0 & 51 & 0 \\
\hline & & & $\%$ & $0,0 \%$ & $100,0 \%$ & $0,0 \%$ & $100,0 \%$ & $0,0 \%$ \\
\hline & \multirow{2}{*}{$\begin{array}{l}\text { Premezclas } \\
\text { panificados dulces }\end{array}$} & 16 & $n$ & 0 & 16 & 0 & 16 & 0 \\
\hline & & & $\%$ & $0,0 \%$ & $100,0 \%$ & $0,0 \%$ & $100,0 \%$ & $0,0 \%$ \\
\hline & Barras de cereal con & 12 & $\mathrm{n}$ & 0 & 12 & 0 & 12 & 0 \\
\hline & azúcar & & $\%$ & $0,0 \%$ & $100,0 \%$ & $0,0 \%$ & $100,0 \%$ & $0,0 \%$ \\
\hline & Cereales de & 63 & $\mathrm{n}$ & 0 & 63 & 0 & 60 & 3 \\
\hline & $\begin{array}{l}\text { desayuno con } \\
\text { azúcar }\end{array}$ & & $\%$ & $0,0 \%$ & $100,0 \%$ & $0,0 \%$ & $95,2 \%$ & $4,8 \%$ \\
\hline & Snacks y productos & 80 & $\mathrm{n}$ & 0 & 80 & 0 & 80 & 0 \\
\hline & de copetin & & $\%$ & $0,0 \%$ & $100,0 \%$ & $0,0 \%$ & $100,0 \%$ & $0,0 \%$ \\
\hline & Comidas rápidas & 55 & $\mathrm{n}$ & 0 & 55 & 12 & 42 & 1 \\
\hline & & & $\%$ & $0,0 \%$ & $100,0 \%$ & $21,8 \%$ & $76,4 \%$ & $1,8 \%$ \\
\hline & Aderezos & 57 & $\mathrm{n}$ & 0 & 57 & 0 & 50 & 7 \\
\hline & & & $\%$ & $0,0 \%$ & $100,0 \%$ & $0,0 \%$ & $87,7 \%$ & $12,3 \%$ \\
\hline & Salsas comerciales & 18 & $\mathrm{n}$ & 0 & 18 & 5 & 12 & 1 \\
\hline & & & $\%$ & $0,0 \%$ & $100,0 \%$ & $27,8 \%$ & $66,7 \%$ & $5,6 \%$ \\
\hline & Caldos, & 39 & $n$ & 0 & 39 & 24 & 15 & 0 \\
\hline & $\begin{array}{l}\text { saborizadores y } \\
\text { sopas comerciales }\end{array}$ & & $\%$ & $0,0 \%$ & $100,0 \%$ & $61,5 \%$ & $38,5 \%$ & $0,0 \%$ \\
\hline T & & 1061 & $\mathrm{n}$ & 0 & 1061 & 118 & 886 & 57 \\
\hline lotal & & & $\%$ & $0,0 \%$ & $100,0 \%$ & $11,1 \%$ & $83,5 \%$ & $5,4 \%$ \\
\hline Bebidas sin & Soda & 1 & $\mathrm{n}$ & 1 & 0 & 1 & 0 & 0 \\
\hline azucar & & & $\%$ & $100,0 \%$ & $0,0 \%$ & $100,0 \%$ & $0,0 \%$ & $0,0 \%$ \\
\hline & Aguas minerales & 7 & $\mathrm{n}$ & 7 & 0 & 7 & 0 & 0 \\
\hline & & & $\%$ & $100,0 \%$ & $0,0 \%$ & $100,0 \%$ & $0,0 \%$ & $0,0 \%$ \\
\hline & Infusiones & 1 & $\mathrm{n}$ & 1 & 0 & 1 & 0 & 0 \\
\hline
\end{tabular}




\begin{tabular}{|c|c|c|c|c|c|c|c|c|}
\hline & & & $\%$ & $100,0 \%$ & $0,0 \%$ & $100,0 \%$ & $0,0 \%$ & $0,0 \%$ \\
\hline & \multirow{2}{*}{$\begin{array}{l}\text { Bebidas } \\
\text { edulcoradas }\end{array}$} & \multirow[t]{2}{*}{33} & $\mathrm{n}$ & 33 & 0 & 32 & 0 & 1 \\
\hline & & & $\%$ & $100,0 \%$ & $0,0 \%$ & $97,0 \%$ & $0,0 \%$ & $3,0 \%$ \\
\hline \multirow{2}{*}{\multicolumn{2}{|c|}{ Total }} & \multirow[t]{2}{*}{42} & $\mathrm{n}$ & 42 & 0 & 41 & 0 & 1 \\
\hline & & & $\%$ & $100,0 \%$ & $0,0 \%$ & $97,6 \%$ & $0,0 \%$ & $2,4 \%$ \\
\hline \multirow{2}{*}{\multicolumn{2}{|c|}{ Comidas pre-elaboradas }} & \multirow[t]{2}{*}{11} & $\mathrm{n}$ & 11 & 0 & 6 & 5 & 0 \\
\hline & & & $\%$ & $100,0 \%$ & $0,0 \%$ & $54,5 \%$ & $45,5 \%$ & $0,0 \%$ \\
\hline \multicolumn{2}{|l|}{ Muestra Total } & 1703 & $\mathrm{n}$ & 490 & 1213 & 284 & 1350 & 69 \\
\hline
\end{tabular}

* Alimentos que no pudieron clasificarse por falta de información.

Fuente: Elaboración Propia.

\section{Perfil Ecuador y GAPA}

Como se mencionó en el apartado anterior el Perfil de Ecuador tiene un nivel de acuerdo moderado con las GAPA (Gwet's AC1=0,410) (Tabla 4). En la Tabla 10 se observa un mayor acuerdo en el grupo carnes y huevos (Gwet's $\mathrm{ACl}=0,743)$ y en frutas y verduras (Gwet's $\mathrm{ACl}=$ 0,636) con un acuerdo sustancial. En alimentos de consumo opcional (Gwet's AC1=0,556) presentó un acuerdo moderado y en legumbres, cereales, papa, pan y pastas (Gwet's $\mathrm{AC} 1=0,282)$ un acuerdo discreto. No se encontró acuerdo en el grupo de leche, yogures y quesos (Gwet's AC1=-0,383).

En los grupos donde no se pudo calcular el Índice Gwet's AC1 ni el Kappa, el porcentaje de acuerdo muestra un acuerdo casi perfecto en el grupo comidas pre-elaboradas y en el de grupo aceite crudo, frutos secos y semillas, y un acuerdo moderado en bebidas sin azúcar.

Tabla 10: Nivel de acuerdo entre el perfil de Ecuador y las GAPA por grupos de alimentos $(\mathrm{n}=1579)$

\begin{tabular}{|c|c|c|c|c|c|c|c|c|c|c|c|c|c|}
\hline \multirow{3}{*}{$\begin{array}{l}\text { Grupo de } \\
\text { alimento }\end{array}$} & \multirow{3}{*}{$\begin{array}{l}\text { Mue } \\
\text { stra } \\
\text { total } \\
\text { (n) }\end{array}$} & \multirow{3}{*}{$\begin{array}{c}\text { Clasificacio } \\
\text { n Perfil } \\
\text { Ecuador }\end{array}$} & \multicolumn{6}{|c|}{ Clasificación GAPA } & \multicolumn{5}{|c|}{ Nivel de acuerdo* } \\
\hline & & & \multicolumn{2}{|c|}{$\begin{array}{c}\text { A } \\
\text { promov } \\
\text { er }\end{array}$} & \multicolumn{2}{|c|}{$\begin{array}{l}\text { Consumo } \\
\text { moderado }\end{array}$} & \multicolumn{2}{|c|}{$\underset{\text { limitar }}{\mathrm{A}}$} & \multirow[t]{2}{*}{$\begin{array}{c}\text { Indicad } \\
\text { or }\end{array}$} & \multirow[t]{2}{*}{$\begin{array}{l}\text { Estima } \\
\text { ción }\end{array}$} & \multirow{2}{*}{\multicolumn{2}{|c|}{ IC $95 \%$}} & \multirow{2}{*}{$\begin{array}{l}\text { Interpret } \\
\text { ación } \\
\text { probabil } \\
\text { ística }\end{array}$} \\
\hline & & & $\mathrm{N}$ & $\%$ & $\mathrm{~N}$ & $\%$ & $\mathrm{~N}$ & $\%$ & & & & & \\
\hline \multirow{3}{*}{$\begin{array}{c}\text { Legumbre } \\
\text { s, } \\
\text { cereales, } \\
\text { papa, pan } \\
\text { y pastas }\end{array}$} & \multirow{3}{*}{182} & A promover & 4 & $\begin{array}{l}13 \\
\%\end{array}$ & 26 & $20 \%$ & 0 & $\begin{array}{c}0 \\
\%\end{array}$ & $\begin{array}{c}\% \\
\text { acuerd } \\
\text { o }\end{array}$ & 47 & 39 & 54 & $\begin{array}{l}\text { Modera } \\
\text { do }\end{array}$ \\
\hline & & $\begin{array}{l}\text { Consumo } \\
\text { moderado }\end{array}$ & $\begin{array}{l}2 \\
5\end{array}$ & $\begin{array}{l}81 \\
\%\end{array}$ & 71 & $55 \%$ & $\begin{array}{l}1 \\
1\end{array}$ & $\begin{array}{l}52 \\
\%\end{array}$ & Kappa & $-0,018$ & $\begin{array}{l}0,1 \\
32\end{array}$ & $\begin{array}{l}0,0 \\
96\end{array}$ & $\begin{array}{l}\text { Sin } \\
\text { Acuerdo }\end{array}$ \\
\hline & & A limitar & 2 & $6 \%$ & 33 & $25 \%$ & $\begin{array}{l}1 \\
0\end{array}$ & $\begin{array}{l}48 \\
\%\end{array}$ & $\begin{array}{c}\text { Gwet's } \\
\text { AC1 }\end{array}$ & 0,282 & $\begin{array}{c}0,1 \\
71\end{array}$ & $\begin{array}{l}0,3 \\
94\end{array}$ & Discreto \\
\hline \multirow{3}{*}{$\begin{array}{l}\text { Frutas y } \\
\text { verduras }\end{array}$} & \multirow{3}{*}{53} & A promover & $\begin{array}{l}1 \\
2\end{array}$ & $\begin{array}{c}100 \\
\%\end{array}$ & 9 & $32 \%$ & 0 & $\begin{array}{c}0 \\
\%\end{array}$ & $\begin{array}{c}\% \\
\text { acuerd } \\
\text { o }\end{array}$ & 76 & 64 & 87 & $\begin{array}{l}\text { Sustanci } \\
\text { al }\end{array}$ \\
\hline & & $\begin{array}{l}\text { Consumo } \\
\text { moderado }\end{array}$ & 0 & $0 \%$ & 16 & $57 \%$ & 1 & $\begin{array}{c}8 \\
\%\end{array}$ & Kappa & 0,635 & $\begin{array}{c}0,4 \\
63\end{array}$ & $\begin{array}{c}0,8 \\
07\end{array}$ & $\begin{array}{l}\text { Sustanci } \\
\text { al }\end{array}$ \\
\hline & & A limitar & 0 & $0 \%$ & 3 & $11 \%$ & $\begin{array}{l}1 \\
2\end{array}$ & $\begin{array}{l}92 \\
\%\end{array}$ & $\begin{array}{c}\text { Gwet's } \\
\text { AC1 }\end{array}$ & 0,636 & $\begin{array}{l}0,4 \\
59\end{array}$ & $\begin{array}{l}0,8 \\
13\end{array}$ & $\begin{array}{l}\text { Sustanci } \\
\text { al }\end{array}$ \\
\hline
\end{tabular}




\begin{tabular}{|c|c|c|c|c|c|c|c|c|c|c|c|c|c|}
\hline \multirow{3}{*}{$\begin{array}{c}\text { Carnes y } \\
\text { huevo }\end{array}$} & \multirow{3}{*}{135} & A promover & 5 & $\begin{array}{c}100 \\
\%\end{array}$ & 0 & $0 \%$ & 0 & $\begin{array}{c}0 \\
\%\end{array}$ & $\begin{array}{c}\% \\
\text { acuerd } \\
0\end{array}$ & 79 & 72 & 86 & $\begin{array}{l}\text { Sustanci } \\
\text { al }\end{array}$ \\
\hline & & $\begin{array}{l}\text { Consumo } \\
\text { moderado }\end{array}$ & 0 & $0 \%$ & 14 & $93 \%$ & $\begin{array}{l}2 \\
7\end{array}$ & $\begin{array}{l}23 \\
\%\end{array}$ & Kappa & 0,487 & $\begin{array}{l}0,3 \\
33\end{array}$ & $\begin{array}{c}0,6 \\
41\end{array}$ & $\begin{array}{l}\text { Modera } \\
\text { do }\end{array}$ \\
\hline & & A limitar & 0 & $0 \%$ & 1 & $7 \%$ & $\begin{array}{l}8 \\
8\end{array}$ & $\begin{array}{l}77 \\
\%\end{array}$ & $\begin{array}{c}\text { Gwet's } \\
\text { AC1 }\end{array}$ & 0,743 & $\begin{array}{c}0,6 \\
51\end{array}$ & $\begin{array}{c}0,8 \\
35\end{array}$ & $\begin{array}{l}\text { Sustanci } \\
\text { al }\end{array}$ \\
\hline \multirow{3}{*}{$\begin{array}{l}\text { Leche, } \\
\text { yogur y } \\
\text { quesos }\end{array}$} & \multirow{3}{*}{197} & A promover & 4 & $5 \%$ & 0 & $0 \%$ & 0 & $\begin{array}{l}\mathrm{X} * \\
* *\end{array}$ & $\begin{array}{c}\% \\
\text { acuerd } \\
0\end{array}$ & 6 & 3 & 9 & $\begin{array}{l}\text { Insignifi } \\
\text { cante }\end{array}$ \\
\hline & & $\begin{array}{l}\text { Consumo } \\
\text { moderado }\end{array}$ & $\begin{array}{l}2 \\
4\end{array}$ & $\begin{array}{l}31 \\
\%\end{array}$ & 8 & $7 \%$ & 0 & $\mathrm{x}$ & Kappa & $\mathrm{x}$ & $\mathrm{x}$ & $\mathrm{x}$ & $\mathrm{x}$ \\
\hline & & A limitar & $\begin{array}{l}5 \\
0\end{array}$ & $\begin{array}{l}64 \\
\%\end{array}$ & 111 & $93 \%$ & 0 & $\mathrm{x}$ & $\begin{array}{c}\text { Gwet's } \\
\text { AC1 }\end{array}$ & $-0,383$ & $\begin{array}{c}- \\
0,4 \\
35\end{array}$ & $\begin{array}{c}- \\
0,3 \\
31\end{array}$ & $\begin{array}{l}\text { Sin } \\
\text { Acuerdo }\end{array}$ \\
\hline \multirow{3}{*}{$\begin{array}{l}\text { Aceite } \\
\text { crudo, } \\
\text { frutos } \\
\text { secos y } \\
\text { semillas }\end{array}$} & \multirow{3}{*}{8} & A promover & 8 & $\begin{array}{c}100 \\
\%\end{array}$ & 0 & $\mathrm{x}$ & 0 & $\mathrm{x}$ & $\begin{array}{c}\% \\
\text { acuerd } \\
0 \\
\end{array}$ & 100 & $\mathrm{x}$ & $\mathrm{x}$ & Perfecto \\
\hline & & $\begin{array}{l}\text { Consumo } \\
\text { moderado }\end{array}$ & 0 & $0 \%$ & 0 & $\mathrm{x}$ & 0 & $\mathrm{x}$ & Карра & $\mathrm{x}$ & $\mathrm{x}$ & $\mathrm{x}$ & $\mathrm{x}$ \\
\hline & & A limitar & 0 & $0 \%$ & 0 & $\mathrm{x}$ & 0 & $\mathrm{x}$ & $\begin{array}{c}\text { Gwet's } \\
\text { AC1 }\end{array}$ & $\mathrm{x}$ & $\mathrm{X}$ & $\mathrm{x}$ & $\mathrm{x}$ \\
\hline \multirow{3}{*}{$\begin{array}{l}\text { Alimento } \\
\text { s de } \\
\text { consumo } \\
\text { opcional }\end{array}$} & \multirow{3}{*}{991} & A promover & 0 & $\mathrm{x}$ & 0 & $\mathrm{x}$ & 6 & $\begin{array}{c}1 \\
\%\end{array}$ & $\begin{array}{c}\% \\
\text { acuerd } \\
0\end{array}$ & 62 & 59 & 65 & $\begin{array}{l}\text { Sustanci } \\
\text { al }\end{array}$ \\
\hline & & $\begin{array}{l}\text { Consumo } \\
\text { moderado }\end{array}$ & 0 & $\mathrm{x}$ & 0 & $\mathrm{x}$ & $\begin{array}{l}3 \\
7 \\
0\end{array}$ & $\begin{array}{l}37 \\
\%\end{array}$ & Kappa & $\mathrm{x}$ & $\mathrm{x}$ & $\mathrm{x}$ & $\mathrm{x}$ \\
\hline & & A limitar & 0 & $\mathrm{x}$ & 0 & $\mathrm{x}$ & $\begin{array}{l}6 \\
1 \\
5\end{array}$ & $\begin{array}{l}62 \\
\%\end{array}$ & $\begin{array}{c}\text { Gwet's } \\
\text { AC1 }\end{array}$ & 0,556 & $\begin{array}{l}0,5 \\
15\end{array}$ & $\begin{array}{c}0,6 \\
00\end{array}$ & $\begin{array}{l}\text { Modera } \\
\text { do }\end{array}$ \\
\hline \multirow{3}{*}{$\begin{array}{l}\text { Bebidas } \\
\text { sin azúcar }\end{array}$} & \multirow{3}{*}{2} & A promover & 1 & $\begin{array}{c}100 \\
\%\end{array}$ & 1 & $100 \%$ & 0 & $\mathrm{x}$ & $\begin{array}{c}\% \\
\text { acuerd } \\
0\end{array}$ & 50 & $\mathrm{x}$ & $\mathrm{x}$ & $\begin{array}{l}\text { Modera } \\
\text { do }\end{array}$ \\
\hline & & $\begin{array}{l}\text { Consumo } \\
\text { moderado }\end{array}$ & 0 & $0 \%$ & 0 & $0 \%$ & 0 & $\mathrm{x}$ & Kappa & $\mathrm{x}$ & $\mathrm{x}$ & $\mathrm{x}$ & $\mathrm{x}$ \\
\hline & & A limitar & 0 & $0 \%$ & 0 & $0 \%$ & 0 & $\mathrm{x}$ & $\begin{array}{c}\text { Gwet's } \\
\text { AC1 }\end{array}$ & $\mathrm{x}$ & $\mathrm{x}$ & $\mathrm{x}$ & $\mathrm{x}$ \\
\hline \multirow{3}{*}{$\begin{array}{l}\text { Comidas } \\
\text { pre- } \\
\text { elaborada } \\
\text { s }\end{array}$} & \multirow{3}{*}{11} & A promover & 0 & $\mathrm{x}$ & 0 & $0 \%$ & 0 & $\mathrm{x}$ & $\begin{array}{c}\% \\
\text { acuerd } \\
0\end{array}$ & 82 & 54 & 100 & $\begin{array}{l}\text { Casi } \\
\text { Perfecto }\end{array}$ \\
\hline & & $\begin{array}{l}\text { Consumo } \\
\text { moderado }\end{array}$ & 0 & $\mathrm{x}$ & 9 & $82 \%$ & 0 & $\mathrm{x}$ & Kappa & $\mathrm{x}$ & $\mathrm{x}$ & $\mathrm{x}$ & $\mathrm{x}$ \\
\hline & & A limitar & 0 & $\mathrm{x}$ & 2 & $18 \%$ & 0 & $\mathrm{x}$ & $\begin{array}{c}\text { Gwet's } \\
\text { AC1 }\end{array}$ & $\mathrm{x}$ & $\mathrm{x}$ & $\mathrm{x}$ & $\mathrm{x}$ \\
\hline $\begin{array}{l}\text { Muestra } \\
\text { Total** }\end{array}$ & 1579 & & & & & & & & & & & & \\
\hline
\end{tabular}

* En la lectura de la Tabla se reporta solo el Índice Gwet's AC1 por tener mayor robustez científica (Ver metodología)

** Los productos de la categoría Sin dato se han descartado de la muestra total para el análisis del nivel de acuerdo (Ver metodología)

*** No se ha podido realizar el cálculo por la naturaleza de los datos.

Fuente: Elaboración Propia 
En cuanto al nivel de exigencia, en el total de la muestra las GAPA poseen un nivel de exigencia mayor que el perfil de Ecuador (71,2\%vs 54,4\%) (Tabla 2). A nivel de los grupos de alimentos, en la tabla 11 se observa que a las GAPA son menos exigentes que el perfil de Ecuador en todos los grupos excepto en el grupo carnes y huevos (87\% GAPA vs 64,5\% Ecuador), alimentos de consumo opcional (100\% GAPA vs 58\% Ecuador), bebidas sin azúcar ( $0 \%$ GAPA y $0 \%$ Ecuador) y aceite crudo, frutos secos y semillas ( $0 \%$ GAPA y $0 \%$ Ecuador).

En el grupo de legumbres, cereales, papa, pan y pastas, el perfil de Ecuador resulta menos exigente que las GAPA en la categoría de pastas rellenas (100\% GAPA vs 45,5\% Ecuador). En las siguientes categorías las GAPA promueven el 100\% de los productos mientras que el perfil de Ecuador limita algunos de los productos incluidos por su alto contenido en sodio: 5,4\% cereales refinados, $20,8 \%$ pan blanco y $16,7 \%$ pan integral y $41,7 \%$ premezclas de panificados y cereales, y 57,1\% amasados (Tabla 4 ANEXO III y Tabla 11)

En el grupo de frutas y verduras el perfil de Ecuador coincide con las GAPA en limitar las verduras en salmuera (100 GAPA y 92,3\% Ecuador) pero además limita productos de las siguientes categorías por su alto contenido en sodio: $4 \%$ de verduras y frutas enlatadas y $66,7 \%$ de las comidas listas de verdura (Tabla 4 ANEXO III y Tabla 11)

En el grupo de leche, yogur y quesos, difieren en la clasificación de las categorías de quesos, las GAPA promueven su consumo mientras que el perfil de Ecuador clasifica "a limitar" a un $94,9 \%$ de los quesos duros, semiduros y saborizados y un $68,5 \%$ de los quesos blandos, ambos por su alto contenido en sodio y grasas totales (Tabla 4 ANEXO III y Tabla 11)

En comidas pre-elaboradas el perfil de Ecuador clasifica "a limitar" al 18,2\% de los productos por su alto contenido en sodio (Tabla 4 ANEXO III y Tabla 11)

Tabla 11: Nivel de exigencia del perfil de Ecuador y las GAPA por grupos y por categorías de alimentos $(\mathrm{n}=1703)$

\begin{tabular}{|c|c|c|c|c|c|c|c|c|c|c|}
\hline \multirow{2}{*}{$\begin{array}{l}\text { Grupo de } \\
\text { alimento }\end{array}$} & \multirow{2}{*}{$\begin{array}{l}\text { Categorías } \\
\text { de alimentos }\end{array}$} & \multirow[t]{2}{*}{$\mathrm{n}$} & & \multicolumn{3}{|c|}{ Clasificación GAPA } & \multicolumn{4}{|c|}{ Clasificación Perfil Ecuador } \\
\hline & & & & $\begin{array}{c}\text { A } \\
\text { promove } \\
\mathrm{r}\end{array}$ & $\begin{array}{c}\text { Consum } \\
\text { o } \\
\text { moderad } \\
\text { o }\end{array}$ & $\begin{array}{c}\mathrm{A} \\
\text { limitar }\end{array}$ & $\begin{array}{c}\text { A } \\
\text { Promove } \\
r\end{array}$ & $\begin{array}{c}\text { Consum } \\
\text { o } \\
\text { moderad } \\
\text { o }\end{array}$ & $\begin{array}{c}\mathrm{A} \\
\text { Limita } \\
\mathrm{r}\end{array}$ & $\begin{array}{c}\text { Sin } \\
\text { dato* }^{*}\end{array}$ \\
\hline \multirow{8}{*}{$\begin{array}{l}\text { Legumbre } \\
\text { s, cereales, } \\
\text { papa, pan } \\
\text { y pastas }\end{array}$} & \multirow[t]{2}{*}{ Legumbres } & \multirow[t]{2}{*}{2} & $\mathrm{n}$ & 2 & 0 & 0 & 2 & 0 & 0 & 0 \\
\hline & & & $\%$ & $100,0 \%$ & $0,0 \%$ & $0,0 \%$ & $100,0 \%$ & $0,0 \%$ & $0,0 \%$ & $0,0 \%$ \\
\hline & \multirow{2}{*}{$\begin{array}{l}\text { Legumbres } \\
\text { enlatadas }\end{array}$} & \multirow[t]{2}{*}{7} & $\mathrm{n}$ & 0 & 7 & 0 & 0 & 7 & 0 & 0 \\
\hline & & & $\%$ & $0,0 \%$ & $100,0 \%$ & $0,0 \%$ & $0,0 \%$ & $100,0 \%$ & $0,0 \%$ & $0,0 \%$ \\
\hline & \multirow{2}{*}{$\begin{array}{l}\text { Cereales } \\
\text { refinados: } \\
\text { arroz, } \\
\text { fideos, } \\
\text { pastas }\end{array}$} & \multirow[t]{2}{*}{37} & $\mathrm{n}$ & 0 & 37 & 0 & 26 & 7 & 2 & 2 \\
\hline & & & $\%$ & $0,0 \%$ & $100,0 \%$ & $0,0 \%$ & $70,3 \%$ & $18,9 \%$ & $5,4 \%$ & $5,4 \%$ \\
\hline & \multirow{2}{*}{$\begin{array}{l}\text { Cereales } \\
\text { integrales }\end{array}$} & \multirow[t]{2}{*}{15} & $\mathrm{n}$ & 15 & 0 & 0 & 0 & 15 & 0 & 0 \\
\hline & & & $\%$ & $100,0 \%$ & $0,0 \%$ & $0,0 \%$ & $0,0 \%$ & $100,0 \%$ & $0,0 \%$ & $0,0 \%$ \\
\hline
\end{tabular}




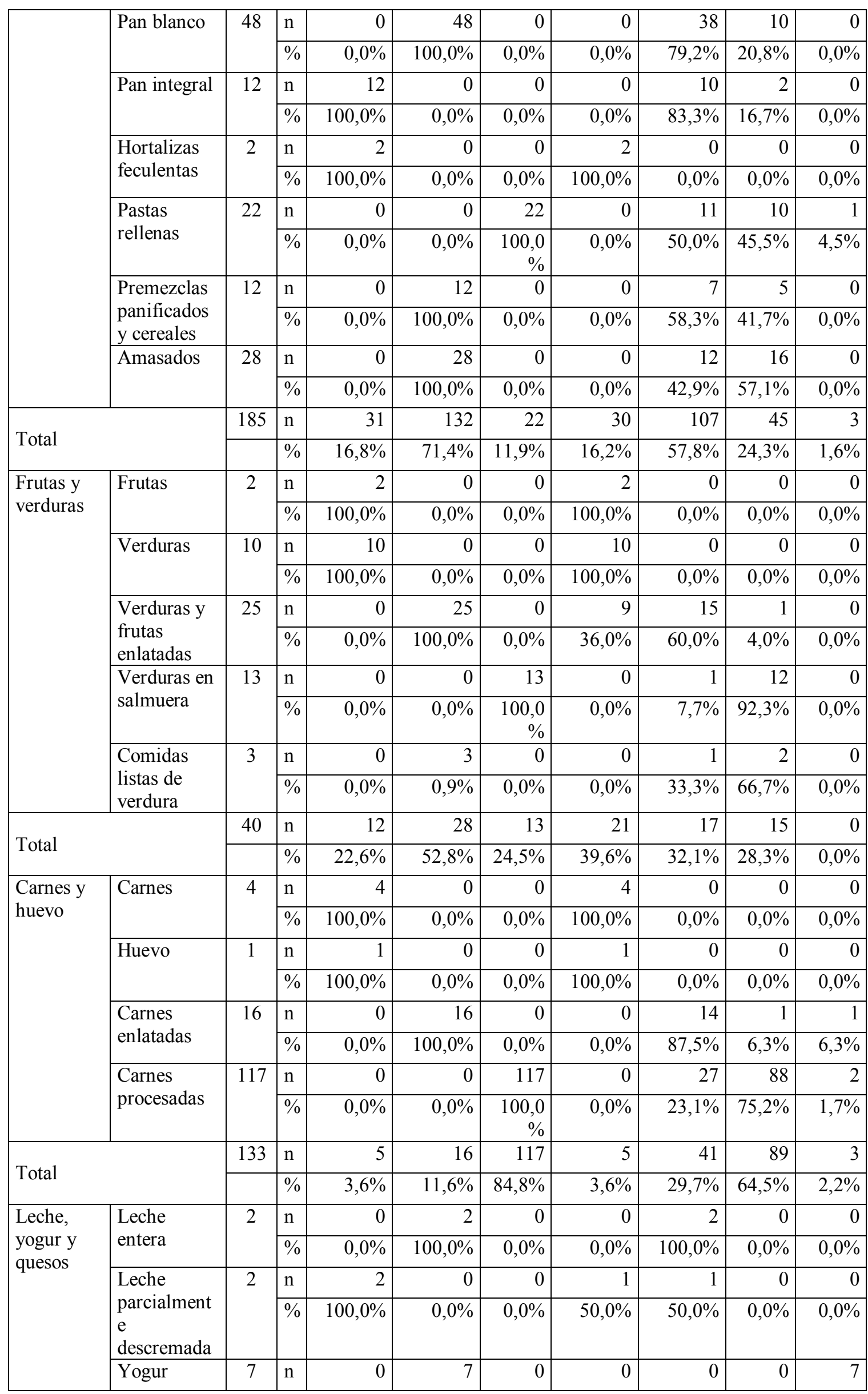




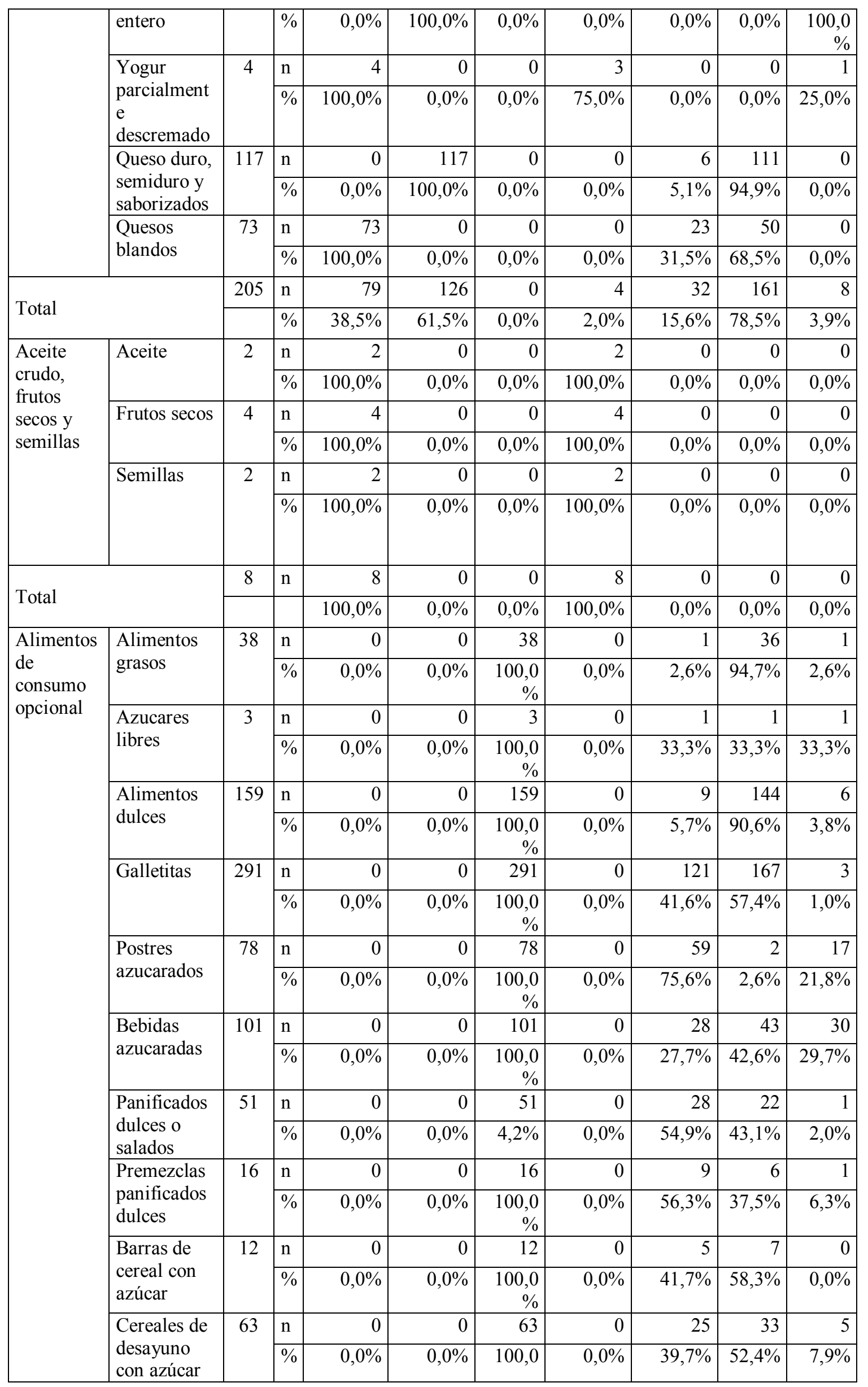




\begin{tabular}{|c|c|c|c|c|c|c|c|c|c|c|}
\hline & & & & & & $\%$ & & & & \\
\hline & Snacks y & 80 & $\mathrm{n}$ & 0 & 0 & 80 & 1 & 2 & 77 & 0 \\
\hline & $\begin{array}{l}\text { productos } \\
\text { de copetin }\end{array}$ & & $\%$ & $0,0 \%$ & $0,0 \%$ & $\begin{array}{r}100,0 \\
\%\end{array}$ & $1,3 \%$ & $2,5 \%$ & $96,3 \%$ & $0,0 \%$ \\
\hline & Comidas & 55 & $n$ & 0 & 0 & 55 & 2 & 32 & 20 & 1 \\
\hline & & & $\%$ & $0,0 \%$ & $0,0 \%$ & $\begin{array}{r}100,0 \\
\%\end{array}$ & $3,6 \%$ & $58,2 \%$ & $36,4 \%$ & $1,8 \%$ \\
\hline & Aderezos & 57 & $n$ & 0 & 0 & 57 & 0 & 10 & 44 & 3 \\
\hline & & & $\%$ & $0,0 \%$ & $0,0 \%$ & $\begin{array}{r}100,0 \\
\%\end{array}$ & $0,0 \%$ & $17,5 \%$ & $77,2 \%$ & $5,3 \%$ \\
\hline & Salsas & 18 & $n$ & 0 & 0 & 18 & 3 & 14 & 1 & 0 \\
\hline & comerciales & & $\%$ & $0,0 \%$ & $0,0 \%$ & $\begin{array}{r}100,0 \\
\%\end{array}$ & $16,7 \%$ & $77,8 \%$ & $5,6 \%$ & $0,0 \%$ \\
\hline & Caldos, & 39 & $n$ & 0 & 0 & 39 & 0 & 26 & 12 & 1 \\
\hline & $\begin{array}{l}\text { saborizadore } \\
\text { s y sopas } \\
\text { comerciales }\end{array}$ & & $\%$ & $0,0 \%$ & $0,0 \%$ & $\begin{array}{r}100,0 \\
\%\end{array}$ & $0,0 \%$ & $66,7 \%$ & $30,8 \%$ & $2,6 \%$ \\
\hline Totol & & $\begin{array}{c}106 \\
1\end{array}$ & $n$ & 0 & 0 & 1061 & 6 & 370 & 615 & 70 \\
\hline lotal & & & $\%$ & $0,0 \%$ & $0,0 \%$ & $\begin{array}{r}100,0 \\
\%\end{array}$ & $0,6 \%$ & $34,9 \%$ & $58,0 \%$ & $6,6 \%$ \\
\hline Bebidas & Soda & 1 & $\mathrm{n}$ & 1 & 0 & 0 & 0 & 0 & 0 & 1 \\
\hline sin azucar & & & $\%$ & $100,0 \%$ & $0,0 \%$ & $0,0 \%$ & $0,0 \%$ & $0,0 \%$ & $0,0 \%$ & $\begin{array}{r}100,0 \\
\%\end{array}$ \\
\hline & Aguas & 7 & $\mathrm{n}$ & 7 & 0 & 0 & 0 & 0 & 0 & 7 \\
\hline & minerales & & $\%$ & $100,0 \%$ & $0,0 \%$ & $0,0 \%$ & $0,0 \%$ & $0,0 \%$ & $0,0 \%$ & $\begin{array}{r}100,0 \\
\%\end{array}$ \\
\hline & Infusiones & 1 & $\mathrm{n}$ & 1 & 0 & 0 & 1 & 0 & 0 & 0 \\
\hline & & & $\%$ & $100,0 \%$ & $0,0 \%$ & $0,0 \%$ & $100,0 \%$ & $0,0 \%$ & $0,0 \%$ & $0,0 \%$ \\
\hline & Bebidas & 33 & $n$ & 0 & 33 & 0 & 1 & 0 & 0 & 32 \\
\hline & & & $\%$ & $0,0 \%$ & $100,0 \%$ & $0,0 \%$ & $3,0 \%$ & $0,0 \%$ & $0,0 \%$ & $97,0 \%$ \\
\hline Tat & & 42 & $\mathrm{n}$ & 9 & 33 & 0 & 2 & 0 & 0 & 40 \\
\hline 10 tal & & & $\%$ & $21,4 \%$ & $78,6 \%$ & $0,0 \%$ & $4,8 \%$ & $0,0 \%$ & $0,0 \%$ & $95,2 \%$ \\
\hline Comidas p 1 & e-elaboradas & 11 & $n$ & 0 & 11 & 0 & 0 & 9 & 2 & 0 \\
\hline & & & $\%$ & $0,0 \%$ & $100,0 \%$ & $0,0 \%$ & $0,0 \%$ & $81,8 \%$ & $18,2 \%$ & $0,0 \%$ \\
\hline Muestra Tc & & $\begin{array}{c}170 \\
3 \\
\end{array}$ & $n$ & 144 & 346 & 1213 & 76 & 576 & 927 & 124 \\
\hline
\end{tabular}

* Alimentos que no pudieron clasificarse por falta de información.

Fuente: Elaboración Propia.

\section{Perfil Perú y GAPA}

Como se mencionó en el apartado anterior el Perfil de Perú tiene un nivel de acuerdo discreto con las GAPA (Gwet's AC1=0,373) (Tabla 4). La Tabla 12 muestra que el perfil de Perú presentó un mayor acuerdo con las GAPA en el grupo de frutas y verduras (Gwet's $\mathrm{AC} 1=0,820$ ) con un acuerdo casi perfecto. Luego en el grupo legumbres, cereales, papa, pan y pastas (Gwet's $A C 1=0,723)$, carnes y huevo (Gwet's $A C 1=0,642)$ y alimentos de consumo opcional (Gwet's $\mathrm{AC} 1=0,601)$ con un acuerdo sustancial. No se encontró acuerdo en el grupo de leche, yogures y quesos (Gwet's $\mathrm{AC} 1=-0,844)$. 
En los grupos donde no se pudo calcular el Índice Gwet's AC1 ni el Kappa, el porcentaje de acuerdo muestra un acuerdo perfecto en el grupo comidas pre-elaboradas, aceite crudo, frutos secos y semillas y en bebidas sin azúcar.

Tabla 12: Nivel de acuerdo entre el perfil de Perú y las GAPA por grupos de alimentos $(\mathrm{n}=1488)$

\begin{tabular}{|c|c|c|c|c|c|c|c|c|c|c|c|}
\hline \multirow{3}{*}{$\begin{array}{l}\text { Grupo de } \\
\text { alimento }\end{array}$} & \multirow{3}{*}{$\mathrm{n}$} & \multirow{3}{*}{$\begin{array}{c}\text { Clasificaci } \\
\text { on Perfil } \\
\text { Perú }\end{array}$} & \multicolumn{4}{|c|}{ Clasificacion GAPA } & \multicolumn{5}{|c|}{ Nivel de acuerdo* } \\
\hline & & & \multicolumn{2}{|c|}{$\begin{array}{c}\text { A } \\
\text { promover }\end{array}$} & \multicolumn{2}{|c|}{$\begin{array}{c}\mathrm{A} \\
\text { limitar }\end{array}$} & \multirow{2}{*}{ Indicador } & \multirow{2}{*}{$\begin{array}{c}\text { Estimaci } \\
\text { ón }\end{array}$} & \multirow{2}{*}{\multicolumn{2}{|c|}{ IC 95\% }} & \multirow{2}{*}{$\begin{array}{l}\text { Interpretaci } \\
\text { ón } \\
\text { probabilísti } \\
\text { ca }\end{array}$} \\
\hline & & & $\mathrm{N}$ & $\%$ & $\mathrm{~N}$ & $\%$ & & & & & \\
\hline \multirow{3}{*}{$\begin{array}{c}\text { Legumbres, } \\
\text { cereales, } \\
\text { papa, pan y } \\
\text { pastas }\end{array}$} & \multirow{3}{*}{180} & $\begin{array}{l}\text { A } \\
\text { promover }\end{array}$ & $\begin{array}{c}13 \\
9\end{array}$ & $87 \%$ & 19 & $\begin{array}{l}90 \\
\%\end{array}$ & $\begin{array}{c}\% \\
\text { acuerdo }\end{array}$ & 78 & 72 & 85 & Sustancial \\
\hline & & A limitar & 20 & $13 \%$ & 2 & $\begin{array}{l}10 \\
\%\end{array}$ & Kappa & $-0,022$ & $\begin{array}{c}- \\
0,16 \\
0\end{array}$ & $\begin{array}{c}0,11 \\
7\end{array}$ & $\begin{array}{l}\text { Sin } \\
\text { Acuerdo }\end{array}$ \\
\hline & & & & & & & $\begin{array}{c}\text { Gwet's } \\
\text { AC1 }\end{array}$ & 0,723 & $\begin{array}{c}0,63 \\
0\end{array}$ & $\begin{array}{c}0,81 \\
9\end{array}$ & Sustancial \\
\hline \multirow{3}{*}{$\begin{array}{l}\text { Frutas y } \\
\text { verduras }\end{array}$} & \multirow{3}{*}{53} & $\begin{array}{l}\text { A } \\
\text { promover }\end{array}$ & 37 & $93 \%$ & 3 & $\begin{array}{l}23 \\
\%\end{array}$ & $\begin{array}{c}\% \\
\text { acuerdo }\end{array}$ & 89 & 80 & 98 & $\begin{array}{l}\text { Casi } \\
\text { Perfecto }\end{array}$ \\
\hline & & A limitar & 3 & $8 \%$ & 10 & $\begin{array}{l}77 \\
\%\end{array}$ & Kappa & 0,694 & $\begin{array}{c}0,45 \\
9\end{array}$ & $\begin{array}{c}0,92 \\
9\end{array}$ & Sustancial \\
\hline & & & & & & & $\begin{array}{l}\text { Gwet's } \\
\text { AC1 }\end{array}$ & 0,820 & $\begin{array}{c}0,67 \\
0\end{array}$ & $\begin{array}{c}0,97 \\
1\end{array}$ & $\begin{array}{l}\text { Casi } \\
\text { Perfecto }\end{array}$ \\
\hline \multirow{3}{*}{$\begin{array}{l}\text { Carnes y } \\
\text { huevo }\end{array}$} & \multirow{3}{*}{131} & $\begin{array}{l}\text { A } \\
\text { promover }\end{array}$ & 17 & $\begin{array}{c}100 \\
\%\end{array}$ & 29 & $\begin{array}{l}25 \\
\%\end{array}$ & $\begin{array}{c}\% \\
\text { acuerdo }\end{array}$ & 78 & 70 & 86 & Sustancial \\
\hline & & A limitar & 0 & $0 \%$ & 85 & $\begin{array}{l}75 \\
\% \\
\end{array}$ & Kappa & 0,442 & $\begin{array}{c}0,29 \\
1 \\
\end{array}$ & $\begin{array}{c}0,59 \\
2 \\
\end{array}$ & Moderado \\
\hline & & & & & & & $\begin{array}{c}\text { Gwet's } \\
\text { AC1 }\end{array}$ & 0,642 & $\begin{array}{c}0,50 \\
5 \\
\end{array}$ & $\begin{array}{c}0,77 \\
9 \\
\end{array}$ & Sustancial \\
\hline \multirow{3}{*}{$\begin{array}{c}\text { Leche, yogur } \\
\text { y quesos }\end{array}$} & \multirow{3}{*}{195} & \begin{tabular}{|l} 
A \\
promover
\end{tabular} & 13 & $7 \%$ & 0 & $\begin{array}{c}\mathrm{X} * * \\
*\end{array}$ & $\begin{array}{c}\% \\
\text { acuerdo }\end{array}$ & 7 & 3 & 10 & $\begin{array}{l}\text { Insignifica } \\
\text { nte }\end{array}$ \\
\hline & & A limitar & $\begin{array}{c}18 \\
2 \\
\end{array}$ & $93 \%$ & 0 & $\mathrm{x}$ & Kappa & 0 & 0 & 0 & $\begin{array}{l}\text { Sin } \\
\text { Acuerdo }\end{array}$ \\
\hline & & & & & & & $\begin{array}{c}\text { Gwet's } \\
\text { AC1 }\end{array}$ & $-0,844$ & $\begin{array}{c}- \\
0,93 \\
5\end{array}$ & $\begin{array}{c}- \\
0,75 \\
2\end{array}$ & $\begin{array}{l}\text { Sin } \\
\text { Acuerdo }\end{array}$ \\
\hline \multirow{3}{*}{$\begin{array}{l}\text { Aceite crudo, } \\
\text { frutos secos y } \\
\text { semillas }\end{array}$} & \multirow{3}{*}{8} & \begin{tabular}{|l} 
A \\
promover
\end{tabular} & 8 & $\begin{array}{c}100 \\
\% \\
\end{array}$ & 0 & $\mathrm{x}$ & $\begin{array}{c}\% \\
\text { acuerdo } \\
\end{array}$ & 100 & $\mathrm{x}$ & $\mathrm{x}$ & Perfecto \\
\hline & & A limitar & 0 & $0 \%$ & 0 & $\mathrm{x}$ & Kappa & $\mathrm{x}$ & $\mathrm{x}$ & $\mathrm{x}$ & $\mathrm{x}$ \\
\hline & & & & & & & $\begin{array}{c}\text { Gwet's } \\
\text { AC1 }\end{array}$ & $\mathrm{x}$ & $\mathrm{x}$ & $\mathrm{x}$ & $\mathrm{X}$ \\
\hline \multirow{3}{*}{$\begin{array}{c}\text { Alimentos de } \\
\text { consumo } \\
\text { opcional }\end{array}$} & \multirow{3}{*}{869} & $\begin{array}{l}\text { A } \\
\text { promover }\end{array}$ & 0 & $\mathrm{x}$ & $\begin{array}{c}27 \\
0 \\
\end{array}$ & $\begin{array}{l}31 \\
\% \\
\end{array}$ & $\begin{array}{c}\% \\
\text { acuerdo }\end{array}$ & 68 & 65 & 73 & Sustancial \\
\hline & & A limitar & 0 & $\mathrm{x}$ & $\begin{array}{c}59 \\
9 \\
\end{array}$ & $\begin{array}{l}69 \\
\%\end{array}$ & Kappa & $\mathrm{X}$ & $\mathrm{x}$ & $\mathrm{x}$ & $\mathrm{x}$ \\
\hline & & & & & & & $\begin{array}{l}\text { Gwet's } \\
\text { AC1 }\end{array}$ & 0,601 & $\begin{array}{c}0,54 \\
9\end{array}$ & $\begin{array}{c}0,65 \\
2\end{array}$ & Sustancial \\
\hline \multirow{3}{*}{$\begin{array}{l}\text { Bebidas sin } \\
\text { azúcar }\end{array}$} & \multirow{3}{*}{41} & $\begin{array}{l}\text { A } \\
\text { promover }\end{array}$ & 41 & $\begin{array}{c}100 \\
\%\end{array}$ & 0 & $\mathrm{x}$ & $\begin{array}{c}\% \\
\text { acuerdo }\end{array}$ & 100 & $\mathrm{x}$ & $\mathrm{x}$ & Perfecto \\
\hline & & A limitar & 0 & $0 \%$ & 0 & $\mathrm{x}$ & Kappa & $\mathrm{x}$ & $\mathrm{x}$ & $\mathrm{x}$ & $\mathrm{x}$ \\
\hline & & & & & & & $\begin{array}{c}\text { Gwet's } \\
\text { AC1 }\end{array}$ & $\mathrm{x}$ & $\mathrm{x}$ & $\mathrm{x}$ & $\mathrm{x}$ \\
\hline $\begin{array}{c}\text { Comidas pre- } \\
\text { elaboradas }\end{array}$ & 11 & $\begin{array}{l}\text { A } \\
\text { promover }\end{array}$ & 11 & $\begin{array}{c}100 \\
\%\end{array}$ & 0 & $\mathrm{x}$ & $\begin{array}{c}\% \\
\text { acuerdo }\end{array}$ & 100 & $\mathrm{x}$ & $\mathrm{x}$ & Perfecto \\
\hline
\end{tabular}




\begin{tabular}{|c|c|c|c|c|c|c|c|c|c|c|c|}
\hline & & A limitar & 0 & $0 \%$ & 0 & $\mathrm{x}$ & Kappa & $\mathrm{x}$ & $\mathrm{x}$ & $\mathrm{x}$ & $\mathrm{x}$ \\
\hline & & & & & & & $\begin{array}{c}\text { Gwet's } \\
\text { ACl }\end{array}$ & $\mathrm{X}$ & $\mathrm{x}$ & $\mathrm{x}$ & $\mathrm{x}$ \\
\hline $\begin{array}{l}\text { Muestra } \\
\text { Total** }\end{array}$ & $\begin{array}{c}148 \\
8 \\
\end{array}$ & & & & & & & & & & \\
\hline
\end{tabular}

* En la lectura de la Tabla se reporta solo el Índice Gwet's AC1 por tener mayor robustez científica (Ver metodología)

** Los productos de la categoría sin dato se han descartado de la muestra total para el análisis del nivel de acuerdo (Ver metodología)

*** No se ha podido realizar el cálculo por la naturaleza de los datos.

Fuente: Elaboración Propia

En cuanto al nivel de exigencia, las GAPA poseen un nivel de exigencia mayor que el perfil de Perú (71,2\% vs 52,9\%) (Tabla 2). En la Tabla 13 se observa que a nivel de los grupos de alimentos, el perfil de Perú y las GAPA tienen el mismo nivel de exigencia en la mayoría de los grupos excepto en alimentos de consumo donde las GAPA son más exigentes (100\% GAPA, $56,5 \%$ Perú) y en el grupo leche yogur y quesos donde el perfil resulta más exigente que las GAPA ( $0 \%$ GAPA, 88,8\% Perú) limitando la mayoría de los quesos por su alto contenido en sodio y grasas saturadas :99,1\% de los quesos duros, semiduros y saborizados y un $90,4 \%$ de los quesos blandos (Tabla 5 ANEXO III y Tabla 13)

Tabla 13: Nivel de exigencia del perfil de Perú y las GAPA por grupos y por categorías de alimentos ( $\mathrm{n}=1703)$

\begin{tabular}{|c|c|c|c|c|c|c|c|c|}
\hline \multirow[t]{2}{*}{$\begin{array}{l}\text { Grupo de } \\
\text { alimento }\end{array}$} & \multirow[t]{2}{*}{$\begin{array}{l}\text { Categoría de } \\
\text { alimentos }\end{array}$} & \multirow[t]{2}{*}{$\mathrm{n}$} & & \multicolumn{2}{|c|}{$\begin{array}{c}\text { Clasificación } \\
\text { GAPA }\end{array}$} & \multicolumn{3}{|c|}{ Clasificación Perfil Perú } \\
\hline & & & & $\begin{array}{c}\text { A } \\
\text { Promover }\end{array}$ & $\begin{array}{c}\mathrm{A} \\
\text { Limitar }\end{array}$ & $\begin{array}{c}\text { A } \\
\text { Promover }\end{array}$ & $\begin{array}{c}\mathrm{A} \\
\text { Limitar }\end{array}$ & $\begin{array}{c}\text { Sin } \\
\text { dato* }\end{array}$ \\
\hline \multirow{17}{*}{$\begin{array}{l}\text { Legumbres, } \\
\text { cereales, papa, } \\
\text { pan y pastas }\end{array}$} & \multirow[t]{2}{*}{ Legumbres } & \multirow[t]{2}{*}{2} & $\mathrm{n}$ & 2 & 0 & 2 & 0 & 0 \\
\hline & & & $\%$ & $100,0 \%$ & $0,0 \%$ & $100,0 \%$ & $0,0 \%$ & $0,0 \%$ \\
\hline & \multirow{2}{*}{$\begin{array}{l}\text { Legumbres } \\
\text { enlatadas }\end{array}$} & \multirow[t]{2}{*}{7} & $\mathrm{n}$ & 7 & 0 & 7 & 0 & 0 \\
\hline & & & $\%$ & $100,0 \%$ & $0,0 \%$ & $100,0 \%$ & $0,0 \%$ & $0,0 \%$ \\
\hline & \multirow{2}{*}{$\begin{array}{l}\text { Cereales refinados: } \\
\text { arroz, fideos, pastas }\end{array}$} & \multirow[t]{2}{*}{37} & $\mathrm{n}$ & 37 & 0 & 35 & 1 & 1 \\
\hline & & & $\%$ & $100,0 \%$ & $0,0 \%$ & $94,6 \%$ & $2,7 \%$ & $2,7 \%$ \\
\hline & \multirow[t]{2}{*}{ Cereales integrales } & \multirow[t]{2}{*}{15} & $\mathrm{n}$ & 15 & 0 & 15 & 0 & 0 \\
\hline & & & $\%$ & $100,0 \%$ & $0,0 \%$ & $100,0 \%$ & $0,0 \%$ & $0,0 \%$ \\
\hline & \multirow[t]{2}{*}{ Pan blanco } & \multirow[t]{2}{*}{48} & $\mathrm{n}$ & 48 & 0 & 46 & 2 & 0 \\
\hline & & & $\%$ & $100,0 \%$ & $0,0 \%$ & $95,8 \%$ & $4,2 \%$ & $0,0 \%$ \\
\hline & \multirow[t]{2}{*}{ Pan integral } & \multirow[t]{2}{*}{12} & $\mathrm{n}$ & 12 & 0 & 11 & 0 & 1 \\
\hline & & & $\%$ & $100,0 \%$ & $0,0 \%$ & $91,7 \%$ & $0,0 \%$ & $8,3 \%$ \\
\hline & \multirow{2}{*}{$\begin{array}{l}\text { Hortalizas } \\
\text { feculentas }\end{array}$} & \multirow[t]{2}{*}{2} & $\mathrm{n}$ & 2 & 0 & 2 & 0 & 0 \\
\hline & & & $\%$ & $100,0 \%$ & $0,0 \%$ & $100,0 \%$ & $0,0 \%$ & $0,0 \%$ \\
\hline & \multirow[t]{2}{*}{ Pastas rellenas } & \multirow[t]{2}{*}{22} & $\mathrm{n}$ & 0 & 22 & 19 & 2 & 1 \\
\hline & & & $\%$ & $0,0 \%$ & $100,0 \%$ & $86,4 \%$ & $9,1 \%$ & $4,5 \%$ \\
\hline & Premezclas & 12 & $\mathrm{n}$ & 12 & 0 & 6 & 6 & 0 \\
\hline
\end{tabular}




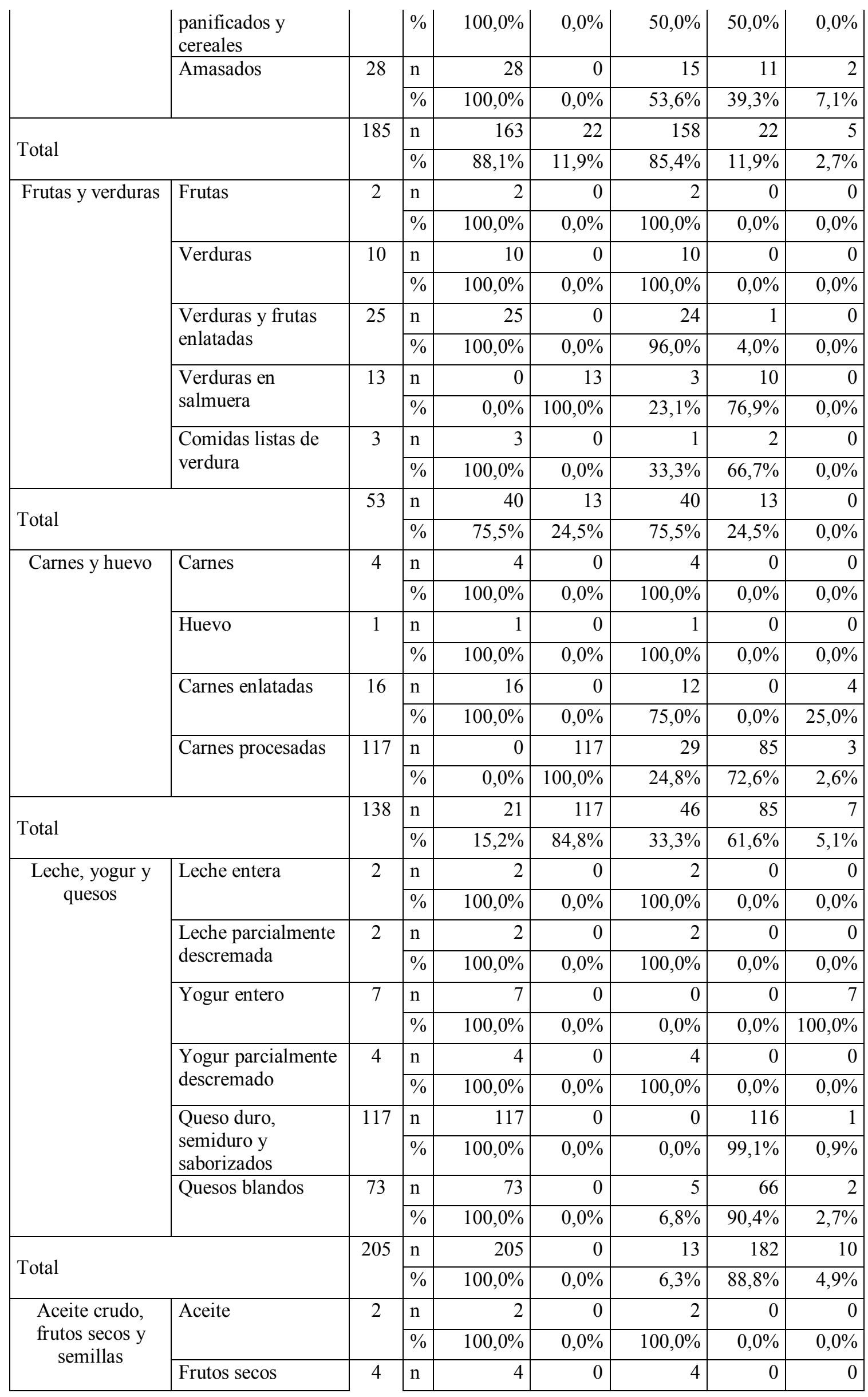




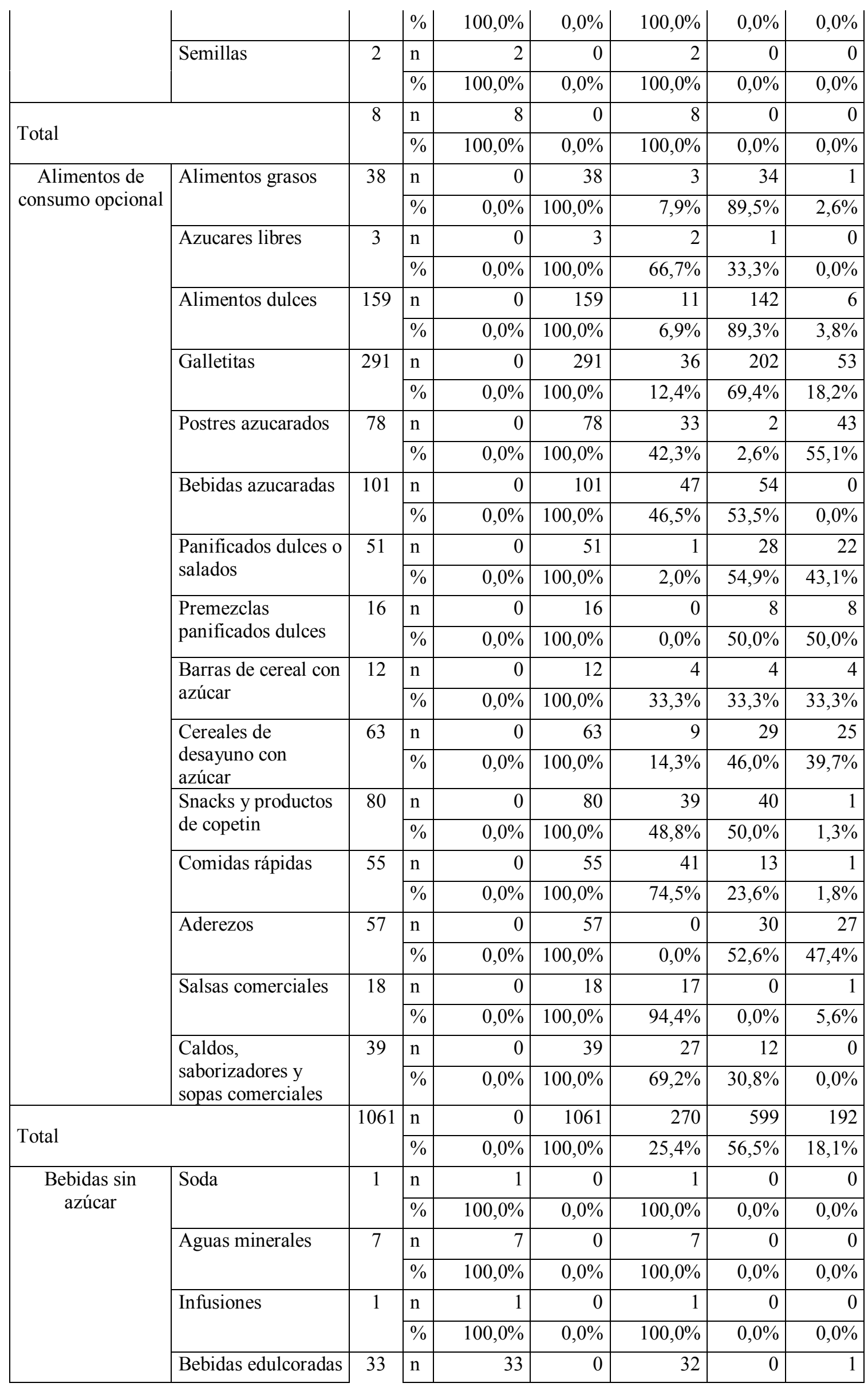




\begin{tabular}{|l|r|l|r|r|r|r|r|r|} 
& & & $\%$ & $100,0 \%$ & $0,0 \%$ & $97,0 \%$ & $0,0 \%$ & $3,0 \%$ \\
\hline \multirow{2}{*}{ Total } & 42 & $\mathrm{n}$ & 42 & 0 & 41 & 0 & 1 \\
\cline { 2 - 9 } & & & $\%$ & $100,0 \%$ & $0,0 \%$ & $97,6 \%$ & $0,0 \%$ & $2,4 \%$ \\
\hline \multirow{2}{*}{ Comidas pre-elaboradas } & 11 & $\mathrm{n}$ & 11 & 0 & 11 & 0 & 0 \\
\cline { 3 - 9 } & & & $100,0 \%$ & $0,0 \%$ & $100,0 \%$ & $0,0 \%$ & $0,0 \%$ \\
\hline Muestra Total & 1703 & $\mathrm{n}$ & 490 & 1213 & 587 & 901 & 215 \\
\hline
\end{tabular}

* Alimentos que no pudieron clasificarse por falta de información.

Fuente: Elaboración Propia.

\section{Perfil Bolivia y GAPA}

El Perfil de Bolivia tiene un nivel de acuerdo discreto con las GAPA (Gwet's AC1=0,350) (Tabla 14). La Tabla 14 muestra que el perfil de Bolivia presenta un mayor acuerdo con las GAPA en el grupo de bebidas sin azúcar (Gwet's AC1=0,759), carnes y huevo (Gwet's AC1= $0,683)$, y frutas y verduras (Gwet's $A C 1=0,636)$ con un acuerdo sustancial. Luego en el grupo alimentos de consumo opcional (Gwet's $\mathrm{AC} 1=0,474)$ con un acuerdo moderado y en legumbres, cereales, papa, pan y pastas (Gwet's $A C 1=0,222)$ con un acuerdo discreto. No se encontró acuerdo en el grupo de leche, yogures y quesos (Gwet's AC1=-0,412).

En los grupos donde no se pudo calcular el Índice Gwet's AC1 ni el Kappa, el porcentaje de acuerdo muestra un acuerdo perfecto en el grupo aceite crudo, frutos secos y semillas y un acuerdo sustancial en comidas pre-elaboradas.

Tabla 14: Nivel de acuerdo entre la clasificación de las GAPA y el Perfil de Bolivia por grupo de alimentos $(\mathrm{n}=1621)$

\begin{tabular}{|c|c|c|c|c|c|c|c|c|c|c|c|c|c|}
\hline \multirow{3}{*}{$\begin{array}{l}\text { Grupo de } \\
\text { alimento }\end{array}$} & \multirow{3}{*}{$\mathrm{n}$} & \multirow{3}{*}{$\begin{array}{c}\text { Clasificacio } \\
\text { n Perfil } \\
\text { Bolivia }\end{array}$} & \multicolumn{6}{|c|}{ Clasificacion GAPA } & \multicolumn{5}{|c|}{ Nivel de acuerdo* } \\
\hline & & & \multicolumn{2}{|c|}{$\begin{array}{c}\text { A } \\
\text { promov } \\
\text { er }\end{array}$} & \multicolumn{2}{|c|}{$\begin{array}{l}\text { Consumo } \\
\text { moderado }\end{array}$} & \multicolumn{2}{|c|}{$\underset{\text { limitar }}{\text { A }}$} & \multirow[t]{2}{*}{$\begin{array}{c}\text { Indicad } \\
\text { or }\end{array}$} & \multirow[t]{2}{*}{$\begin{array}{l}\text { Estima } \\
\text { ción }\end{array}$} & \multirow{2}{*}{\multicolumn{2}{|c|}{ IC 95\% }} & \multirow{2}{*}{$\begin{array}{l}\text { Interpret } \\
\text { ación } \\
\text { probabil } \\
\text { ística }\end{array}$} \\
\hline & & & $\mathrm{N}$ & $\%$ & $\mathrm{~N}$ & $\%$ & $\mathrm{~N}$ & $\%$ & & & & & \\
\hline \multirow{3}{*}{$\begin{array}{c}\text { Legumbre } \\
\text { s, } \\
\text { cereales, } \\
\text { papa, pan } \\
\text { y pastas }\end{array}$} & \multirow{3}{*}{$\begin{array}{c}17 \\
5\end{array}$} & A promover & 9 & $\begin{array}{l}33 \\
\%\end{array}$ & 35 & $28 \%$ & 0 & $\begin{array}{c}0 \\
\%\end{array}$ & $\begin{array}{c}\% \\
\text { acuerd } \\
0\end{array}$ & 44 & 36 & 52 & $\begin{array}{l}\text { Modera } \\
\text { do }\end{array}$ \\
\hline & & $\begin{array}{l}\text { Consumo } \\
\text { moderado }\end{array}$ & $\begin{array}{l}1 \\
6\end{array}$ & $\begin{array}{l}59 \\
\%\end{array}$ & 58 & $46 \%$ & $\begin{array}{l}1 \\
1\end{array}$ & $\begin{array}{l}52 \\
\%\end{array}$ & Kappa & 0,035 & $\begin{array}{c}- \\
0,0 \\
79\end{array}$ & $\begin{array}{c}0,1 \\
48\end{array}$ & $\begin{array}{l}\text { Insignifi } \\
\text { cante }\end{array}$ \\
\hline & & A limitar & 2 & $7 \%$ & 34 & $27 \%$ & $\begin{array}{l}1 \\
0\end{array}$ & $\begin{array}{l}48 \\
\%\end{array}$ & $\begin{array}{c}\text { Gwet's } \\
\text { AC1 }\end{array}$ & 0,222 & $\begin{array}{l}0,1 \\
09\end{array}$ & $\begin{array}{l}0,3 \\
36\end{array}$ & Discreto \\
\hline \multirow{3}{*}{$\begin{array}{l}\text { Frutas y } \\
\text { verduras }\end{array}$} & \multirow{3}{*}{53} & A promover & $\begin{array}{l}1 \\
2\end{array}$ & $\begin{array}{c}100 \\
\%\end{array}$ & 9 & $32 \%$ & 0 & $\begin{array}{c}0 \\
\%\end{array}$ & $\begin{array}{c}\% \\
\text { acuerd } \\
0\end{array}$ & 76 & 64 & 87 & $\begin{array}{l}\text { Sustanci } \\
\text { al }\end{array}$ \\
\hline & & $\begin{array}{l}\text { Consumo } \\
\text { moderado }\end{array}$ & 0 & $0 \%$ & 16 & $57 \%$ & 1 & $\begin{array}{c}8 \\
\%\end{array}$ & Kappa & 0,635 & $\begin{array}{c}0,4 \\
63\end{array}$ & $\begin{array}{l}0,8 \\
07\end{array}$ & $\begin{array}{l}\text { Sustanci } \\
\text { al }\end{array}$ \\
\hline & & A limitar & 0 & $0 \%$ & 3 & $11 \%$ & $\begin{array}{l}1 \\
2\end{array}$ & $\begin{array}{l}92 \\
\%\end{array}$ & $\begin{array}{c}\text { Gwet's } \\
\text { AC1 }\end{array}$ & 0,636 & $\begin{array}{c}0,4 \\
59\end{array}$ & $\begin{array}{l}0,8 \\
13\end{array}$ & $\begin{array}{l}\text { Sustanci } \\
\text { al }\end{array}$ \\
\hline $\begin{array}{c}\text { Carnes y } \\
\text { huevo }\end{array}$ & $\begin{array}{c}13 \\
5\end{array}$ & A promover & 5 & $\begin{array}{c}100 \\
\%\end{array}$ & 0 & $0 \%$ & 0 & $\begin{array}{c}0 \\
\%\end{array}$ & $\begin{array}{c}\% \\
\text { acuerd } \\
0\end{array}$ & 75 & 67 & 82 & $\begin{array}{l}\text { Sustanci } \\
\text { al }\end{array}$ \\
\hline
\end{tabular}




\begin{tabular}{|c|c|c|c|c|c|c|c|c|c|c|c|c|c|}
\hline & & $\begin{array}{l}\text { Consumo } \\
\text { moderado }\end{array}$ & 0 & $0 \%$ & 13 & $93 \%$ & $\begin{array}{l}3 \\
3\end{array}$ & $\begin{array}{l}28 \\
\%\end{array}$ & Kappa & 0,417 & $\begin{array}{c}0,2 \\
69\end{array}$ & $\begin{array}{l}0,5 \\
64\end{array}$ & $\begin{array}{l}\text { Modera } \\
\text { do }\end{array}$ \\
\hline & & A limitar & 0 & $0 \%$ & 1 & $7 \%$ & $\begin{array}{l}8 \\
3 \\
\end{array}$ & $\begin{array}{l}72 \\
\% \\
\end{array}$ & $\begin{array}{c}\text { Gwet's } \\
\text { AC1 }\end{array}$ & 0,683 & $\begin{array}{l}0,5 \\
83 \\
\end{array}$ & $\begin{array}{l}0,7 \\
84 \\
\end{array}$ & $\begin{array}{l}\text { Sustanci } \\
\text { al }\end{array}$ \\
\hline \multirow{3}{*}{$\begin{array}{l}\text { Leche, } \\
\text { yogur y } \\
\text { quesos }\end{array}$} & \multirow{3}{*}{$\begin{array}{c}19 \\
8\end{array}$} & A promover & 3 & $4 \%$ & 0 & $0 \%$ & 0 & $\begin{array}{l}\mathrm{X}^{*} \\
* *\end{array}$ & $\begin{array}{c}\% \\
\text { acuerd } \\
0 \\
\end{array}$ & 4 & 1 & 7 & $\begin{array}{l}\text { Insignifi } \\
\text { cante }\end{array}$ \\
\hline & & $\begin{array}{l}\text { Consumo } \\
\text { moderado }\end{array}$ & $\begin{array}{l}2 \\
3\end{array}$ & $\begin{array}{l}29 \\
\%\end{array}$ & 5 & $4 \%$ & 0 & $\mathrm{x}$ & Kappa & $-0,058$ & $\begin{array}{c} \\
0,0 \\
91 \\
\end{array}$ & $\begin{array}{c}- \\
0,0 \\
24 \\
\end{array}$ & $\begin{array}{l}\text { Sin } \\
\text { Acuerdo }\end{array}$ \\
\hline & & A limitar & $\begin{array}{l}5 \\
2\end{array}$ & $\begin{array}{l}67 \\
\%\end{array}$ & 115 & $96 \%$ & 0 & $\mathrm{x}$ & $\begin{array}{c}\text { Gwet's } \\
\text { AC1 }\end{array}$ & $-0,412$ & $\begin{array}{c}- \\
0,4 \\
55\end{array}$ & $\begin{array}{c}- \\
0,3 \\
7\end{array}$ & $\begin{array}{l}\text { Sin } \\
\text { Acuerdo }\end{array}$ \\
\hline \multirow{3}{*}{$\begin{array}{l}\text { Aceite } \\
\text { crudo, } \\
\text { frutos } \\
\text { secos y } \\
\text { semillas }\end{array}$} & \multirow{3}{*}{8} & A promover & 7 & $\begin{array}{l}88 \\
\%\end{array}$ & 0 & $\mathrm{x}$ & 0 & $\mathrm{x}$ & $\begin{array}{c}\% \\
\text { acuerd } \\
0 \\
\end{array}$ & 88 & 58 & 100 & $\begin{array}{l}\text { Casi } \\
\text { Perfecto }\end{array}$ \\
\hline & & $\begin{array}{l}\text { Consumo } \\
\text { moderado }\end{array}$ & 1 & $\begin{array}{l}13 \\
\%\end{array}$ & 0 & $\mathrm{x}$ & 0 & $\mathrm{x}$ & Kappa & $\mathrm{x}$ & $\mathrm{x}$ & $\mathrm{x}$ & $\mathrm{x}$ \\
\hline & & A limitar & 0 & $0 \%$ & 0 & $\mathrm{x}$ & 0 & $\mathrm{x}$ & $\begin{array}{c}\text { Gwet's } \\
\text { AC1 }\end{array}$ & $\mathrm{x}$ & $\mathrm{x}$ & $\mathrm{x}$ & $\mathrm{x}$ \\
\hline \multirow{3}{*}{$\begin{array}{l}\text { Alimentos } \\
\text { de } \\
\text { consumo } \\
\text { opcional }\end{array}$} & \multirow{3}{*}{$\begin{array}{c}99 \\
9\end{array}$} & A promover & 0 & $\mathrm{x}$ & 0 & $\mathrm{x}$ & 7 & $\begin{array}{c}1 \\
\%\end{array}$ & $\begin{array}{c}\% \\
\text { acuerd } \\
0 \\
\end{array}$ & 56 & 53 & 59 & $\begin{array}{l}\text { Modera } \\
\text { do }\end{array}$ \\
\hline & & $\begin{array}{l}\text { Consumo } \\
\text { moderado }\end{array}$ & 0 & $\mathrm{x}$ & 0 & $\mathrm{x}$ & $\begin{array}{l}4 \\
3 \\
2\end{array}$ & $\begin{array}{l}43 \\
\%\end{array}$ & Kappa & $\mathrm{x}$ & $\mathrm{x}$ & $\mathrm{x}$ & $\mathrm{x}$ \\
\hline & & A limitar & 0 & $\mathrm{x}$ & 0 & $\mathrm{x}$ & $\begin{array}{l}5 \\
6 \\
0 \\
\end{array}$ & $\begin{array}{l}56 \\
\%\end{array}$ & $\begin{array}{c}\text { Gwet's } \\
\text { AC1 }\end{array}$ & 0,474 & $\begin{array}{l}0,4 \\
31\end{array}$ & $\begin{array}{c}0,5 \\
17\end{array}$ & $\begin{array}{l}\text { Modera } \\
\text { do }\end{array}$ \\
\hline \multirow{3}{*}{$\begin{array}{l}\text { Bebidas } \\
\text { sin azúcar }\end{array}$} & \multirow{3}{*}{42} & A promover & 1 & $\begin{array}{l}11 \\
\%\end{array}$ & 0 & $0 \%$ & 0 & $\mathrm{x}$ & $\begin{array}{c}\% \\
\text { acuerd } \\
0 \\
\end{array}$ & 81 & 69 & 93 & $\begin{array}{l}\text { Casi } \\
\text { Perfecto }\end{array}$ \\
\hline & & $\begin{array}{l}\text { Consumo } \\
\text { moderado }\end{array}$ & 8 & $\begin{array}{l}89 \\
\%\end{array}$ & 33 & $100 \%$ & 0 & $\mathrm{x}$ & Kappa & $\mathrm{x}$ & $\mathrm{x}$ & $\mathrm{x}$ & $\mathrm{x}$ \\
\hline & & A limitar & 0 & $0 \%$ & 0 & $0 \%$ & 0 & $\mathrm{x}$ & $\begin{array}{c}\text { Gwet's } \\
\text { AC1 }\end{array}$ & 0,759 & $\begin{array}{l}0,5 \\
74 \\
\end{array}$ & $\begin{array}{c}0,9 \\
44 \\
\end{array}$ & $\begin{array}{l}\text { Sustanci } \\
\text { al }\end{array}$ \\
\hline \multirow{3}{*}{$\begin{array}{l}\text { Comidas } \\
\text { pre- } \\
\text { elaborada } \\
\text { s }\end{array}$} & \multirow{3}{*}{11} & A promover & 0 & $\mathrm{x}$ & 1 & $9 \%$ & 0 & $\mathrm{x}$ & $\begin{array}{c}\% \\
\text { acuerd } \\
0 \\
\end{array}$ & 73 & 41 & 100 & $\begin{array}{l}\text { Sustanci } \\
\text { al }\end{array}$ \\
\hline & & $\begin{array}{l}\text { Consumo } \\
\text { moderado }\end{array}$ & 0 & $\mathrm{x}$ & 8 & $73 \%$ & 0 & $\mathrm{x}$ & Kappa & $\mathrm{x}$ & $\mathrm{x}$ & $\mathrm{x}$ & $\mathrm{x}$ \\
\hline & & A limitar & 0 & $\mathrm{x}$ & 2 & $18 \%$ & 0 & $\mathrm{x}$ & $\begin{array}{c}\text { Gwet's } \\
\text { AC1 }\end{array}$ & $\mathrm{x}$ & $\mathrm{x}$ & $\mathrm{x}$ & $\mathrm{x}$ \\
\hline $\begin{array}{l}\text { Muestra } \\
\text { Total** }\end{array}$ & $\begin{array}{l}16 \\
21\end{array}$ & & & & & & & & & & & & \\
\hline
\end{tabular}

* En la lectura de la Tabla se reporta solo el Índice Gwet's AC1 por tener mayor robustez científica (Ver metodología)

** Los productos de la categoría sin dato se han descartado de la muestra total para el análisis del nivel de acuerdo (Ver metodología)

*** No se ha podido realizar el cálculo por la naturaleza de los datos.

Fuente: Elaboración Propia 
En cuanto al nivel de exigencia, las GAPA poseen un nivel de exigencia mayor que el perfil de Bolivia (71,2\%vs 51,3\%) (Tabla 2). En la Tabla 15 se observa que a nivel de los grupos de alimentos, las GAPA son más exigentes que el perfil de Bolivia solamente en el grupo carnes y huevos (87\% GAPA vs 60,9\% Bolivia), y en el grupo alimentos de consumo opcional $(100 \%$ GAPA vs 52,8\% Bolivia). En el grupo de aceite crudo, frutos secos y semillas y el grupo bebidas sin azúcar coinciden en promover la totalidad de los productos.

En el grupo de legumbres, cereales, papa, pan y pastas, el perfil de Bolivia es menos exigente que las GAPA en la categoría de pastas rellenas (100\% GAPA vs 45,5\% Bolivia). En las siguientes categorías las GAPA promueven el 100\% de los productos mientras que el perfil de Ecuador limita algunos de los productos incluidos por su alto contenido en sodio: 5,4\% cereales refinados, $20,8 \%$ pan blanco y $16,7 \%$ pan integral y $41,7 \%$ premezclas de panificados y cereales, y 57,1\% amasados (Tabla 6 ANEXO III y Tabla 15).

En el grupo de frutas y verduras el perfil de Bolivia coincide con las GAPA en limitar las verduras en salmuera (100 GAPA y 92,3\% Bolivia) pero además limita productos de las siguientes categorías por su alto contenido en sodio: $4 \%$ de verduras y frutas enlatadas y $66,7 \%$ de las comidas listas de verdura (Tabla 6 ANEXO III y Tabla 15).

En el grupo de leche, yogur y quesos, difieren en la clasificación de las categorías de quesos, las GAPA promueven su consumo mientras que el perfil de Ecuador clasifica a limitar a un $98,3 \%$ de los quesos duros, semiduros y saborizados y un $71,2 \%$ de los quesos blandos, ambos por su alto contenido en sodio y grasas saturadas (Tabla 6 ANEXO III y Tabla 15).

En comidas pre-elaboradas el perfil de Ecuador clasifica a limitar al 18,2\% de los productos por su alto contenido en sodio (Tabla 6 ANEXO III y Tabla 15).

Tabla 15: Nivel de exigencia del perfil de Bolivia y las GAPA por grupos y por categorías de alimentos $(\mathrm{n}=1703)$

\begin{tabular}{|c|c|c|c|c|c|c|c|c|c|c|}
\hline \multirow{2}{*}{$\begin{array}{l}\text { Grupo de } \\
\text { alimento }\end{array}$} & \multirow{2}{*}{$\begin{array}{c}\text { Categorías } \\
\text { de alimentos }\end{array}$} & \multirow[t]{2}{*}{$\mathrm{n}$} & & \multicolumn{3}{|c|}{ Clasificación GAPA } & \multicolumn{4}{|c|}{ Clasificación Perfil Bolivia } \\
\hline & & & & $\begin{array}{c}\mathrm{A} \\
\text { promove } \\
\mathrm{r}\end{array}$ & $\begin{array}{c}\text { Consum } \\
\text { o } \\
\text { moderad } \\
\text { o }\end{array}$ & $\begin{array}{c}\mathrm{A} \\
\text { limitar }\end{array}$ & $\begin{array}{c}\text { A } \\
\text { Promove } \\
\text { r }\end{array}$ & $\begin{array}{c}\text { Consum } \\
\text { o } \\
\text { moderad } \\
\text { o }\end{array}$ & $\begin{array}{c}\text { A } \\
\text { Limita } \\
r\end{array}$ & $\begin{array}{c}\text { Sin } \\
\text { dato* }\end{array}$ \\
\hline \multirow{8}{*}{$\begin{array}{l}\text { Legumbre } \\
\text { s, cereales, } \\
\text { papa, pan } \\
\text { y pastas }\end{array}$} & \multirow[t]{2}{*}{ Legumbres } & \multirow[t]{2}{*}{2} & $\mathrm{n}$ & 2 & 0 & 0 & 2 & 0 & 0 & 0 \\
\hline & & & $\%$ & $100,0 \%$ & $0,0 \%$ & $0,0 \%$ & $100,0 \%$ & $0,0 \%$ & $0,0 \%$ & $0,0 \%$ \\
\hline & \multirow{2}{*}{$\begin{array}{l}\text { Legumbres } \\
\text { enlatadas }\end{array}$} & \multirow[t]{2}{*}{7} & $\mathrm{n}$ & 0 & 7 & 0 & 0 & 7 & 0 & 0 \\
\hline & & & $\%$ & $0,0 \%$ & $100,0 \%$ & $0,0 \%$ & $0,0 \%$ & $100,0 \%$ & $0,0 \%$ & $0,0 \%$ \\
\hline & \multirow{2}{*}{\begin{tabular}{|l|} 
Cereales \\
refinados: \\
arroz, \\
fideos, \\
pastas \\
\end{tabular}} & \multirow[t]{2}{*}{37} & $\mathrm{n}$ & 0 & 37 & 0 & 29 & 4 & 2 & 2 \\
\hline & & & $\%$ & $0,0 \%$ & $100,0 \%$ & $0,0 \%$ & $78,4 \%$ & $10,8 \%$ & $5,4 \%$ & $5,4 \%$ \\
\hline & \multirow{2}{*}{$\begin{array}{l}\text { Cereales } \\
\text { integrales }\end{array}$} & \multirow[t]{2}{*}{15} & $\mathrm{n}$ & 15 & 0 & 0 & 5 & 8 & 0 & 2 \\
\hline & & & $\%$ & $100,0 \%$ & $0,0 \%$ & $0,0 \%$ & $33,3 \%$ & $53,3 \%$ & $0,0 \%$ & $\begin{array}{r}13,3 \\
\% \\
\end{array}$ \\
\hline
\end{tabular}




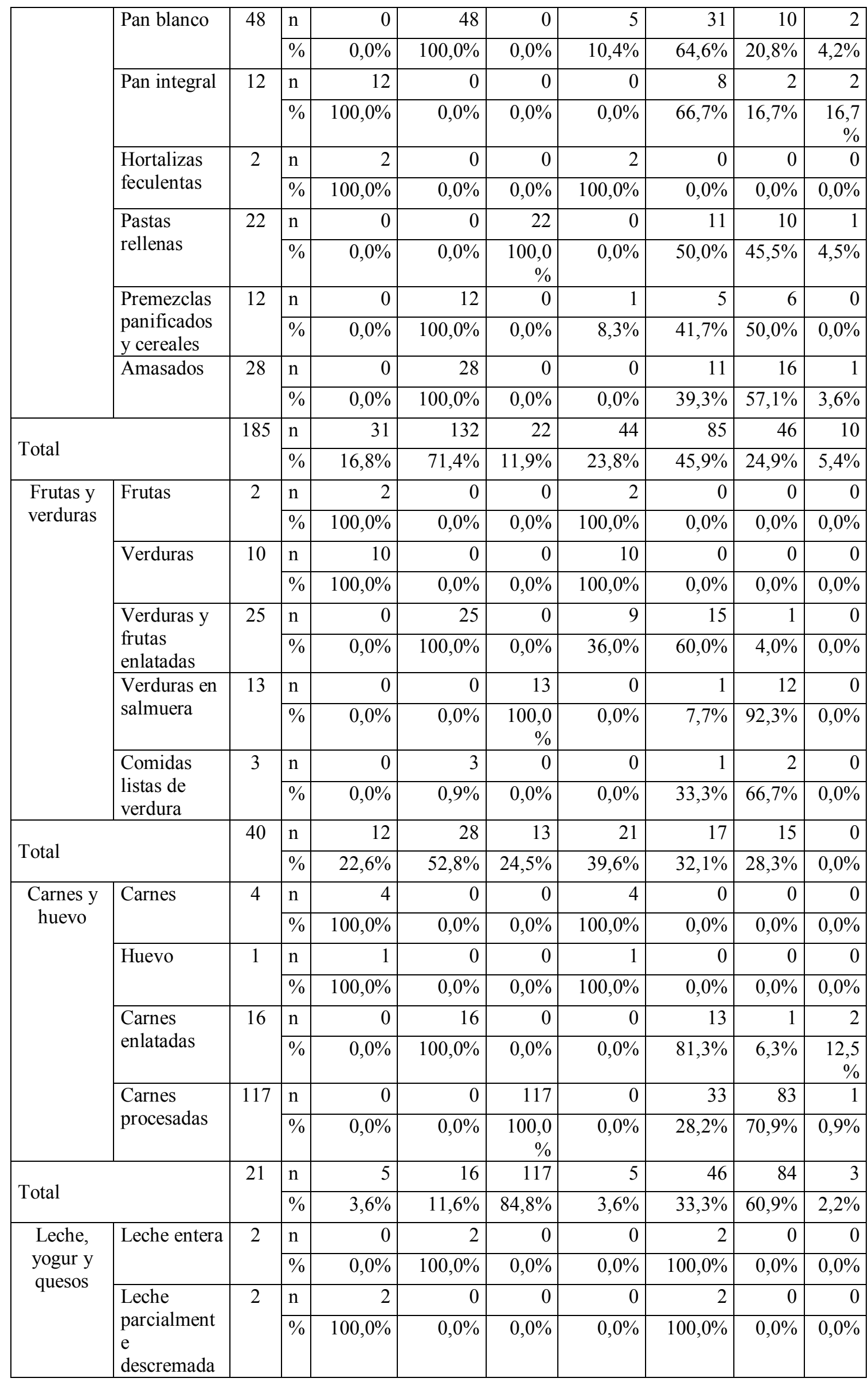




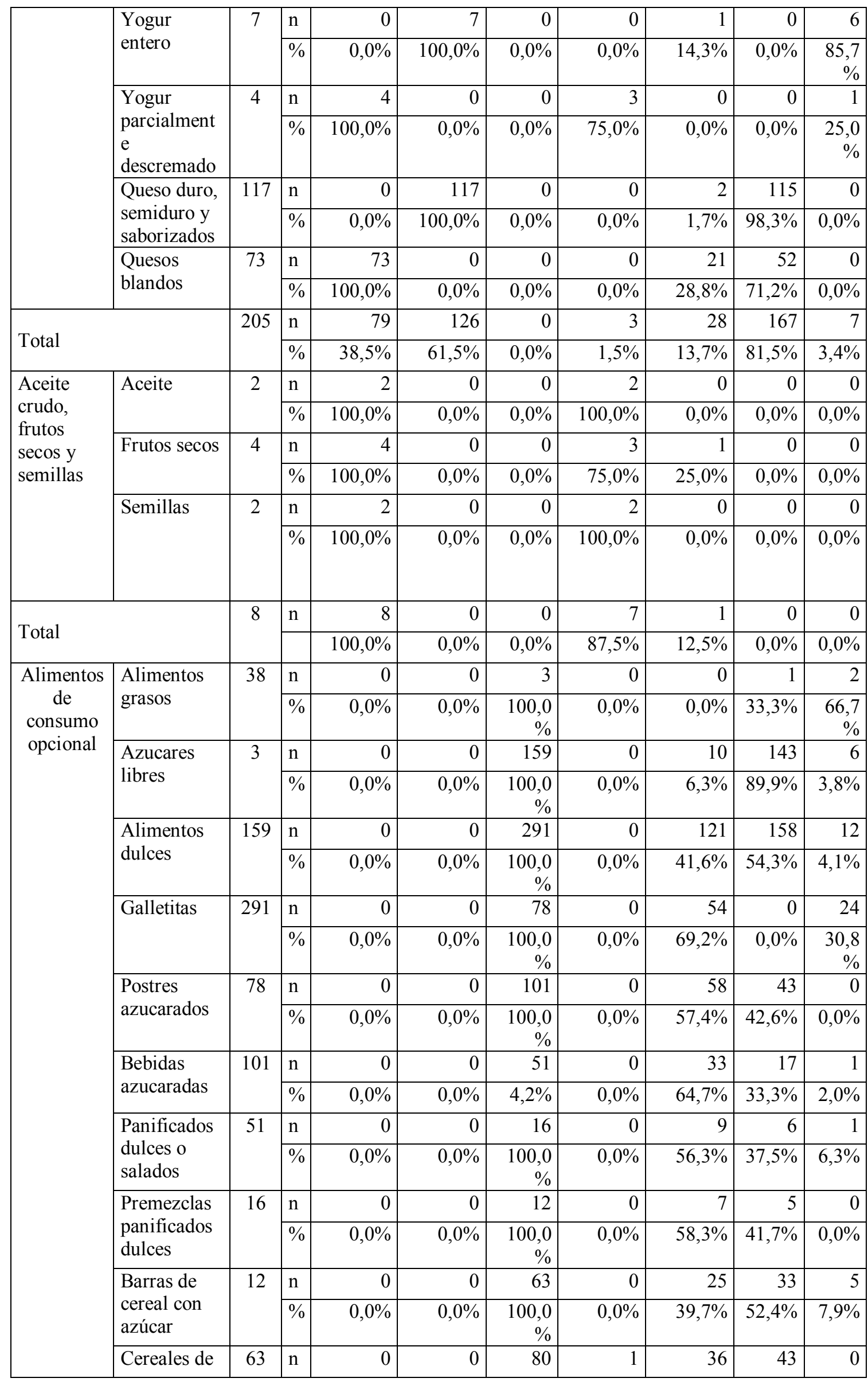




\begin{tabular}{|c|c|c|c|c|c|c|c|c|c|c|}
\hline & $\begin{array}{l}\text { desayuno } \\
\text { con azúcar }\end{array}$ & & $\%$ & $0,0 \%$ & $0,0 \%$ & $\begin{array}{r}100,0 \\
\%\end{array}$ & $1,3 \%$ & $45,0 \%$ & $53,8 \%$ & $0,0 \%$ \\
\hline & Snacks y & 80 & $\mathrm{n}$ & 0 & 0 & 55 & 3 & 28 & 20 & 4 \\
\hline & $\begin{array}{l}\text { productos de } \\
\text { copetin }\end{array}$ & & $\%$ & $0,0 \%$ & $0,0 \%$ & $\begin{array}{r}100,0 \\
0\end{array}$ & $5,5 \%$ & $50,9 \%$ & $36,4 \%$ & $7,3 \%$ \\
\hline & Comidas & 55 & $\mathrm{n}$ & 0 & 0 & 57 & 0 & 10 & 42 & 5 \\
\hline & rápidas & & $\%$ & $0,0 \%$ & $0,0 \%$ & $\begin{array}{r}100,0 \\
\%\end{array}$ & $0,0 \%$ & $17,5 \%$ & $73,7 \%$ & $8,8 \%$ \\
\hline & Aderezos & 57 & $\mathrm{n}$ & 0 & 0 & 18 & 3 & 14 & 1 & 0 \\
\hline & & & $\%$ & $0,0 \%$ & $0,0 \%$ & $\begin{array}{r}100,0 \\
\%\end{array}$ & $16,7 \%$ & $77,8 \%$ & $5,6 \%$ & $0,0 \%$ \\
\hline & Salsas & 18 & $\mathrm{n}$ & 0 & 0 & 39 & 0 & 26 & 12 & 1 \\
\hline & comerciales & & $\%$ & $0,0 \%$ & $0,0 \%$ & $\begin{array}{r}100,0 \\
\%\end{array}$ & $0,0 \%$ & $66,7 \%$ & $30,8 \%$ & $2,6 \%$ \\
\hline & Caldos, & 39 & $\mathrm{n}$ & 0 & 0 & 39 & 0 & 26 & 12 & 1 \\
\hline & $\begin{array}{l}\text { saborizadore } \\
\text { s y sopas } \\
\text { comerciales }\end{array}$ & & $\%$ & $0,0 \%$ & $0,0 \%$ & $\begin{array}{r}100,0 \\
\%\end{array}$ & $0,0 \%$ & $66,7 \%$ & $30,8 \%$ & $2,6 \%$ \\
\hline & & 106 & $\mathrm{n}$ & 0 & 0 & 1061 & 7 & 432 & 560 & 62 \\
\hline Total & & 1 & $\%$ & $0,0 \%$ & $0,0 \%$ & $\begin{array}{r}100,0 \\
\%\end{array}$ & $0,7 \%$ & $40,7 \%$ & $52,8 \%$ & $5,8 \%$ \\
\hline Bebidas & Soda & 1 & $\mathrm{n}$ & 7 & 0 & 0 & 0 & 7 & 0 & 0 \\
\hline $\sin a z$ & & & $\%$ & $100,0 \%$ & $0,0 \%$ & $0,0 \%$ & $0,0 \%$ & $100,0 \%$ & $0,0 \%$ & $0,0 \%$ \\
\hline & Aguas & 7 & $\mathrm{n}$ & 1 & 0 & 0 & 1 & 0 & 0 & 0 \\
\hline & minerales & & $\%$ & $100,0 \%$ & $0,0 \%$ & $0,0 \%$ & $100,0 \%$ & $0,0 \%$ & $0,0 \%$ & $0,0 \%$ \\
\hline & Infusiones & 1 & $\mathrm{n}$ & 0 & 33 & 0 & 0 & 33 & 0 & 0 \\
\hline & & & $\%$ & $0,0 \%$ & $100,0 \%$ & $0,0 \%$ & $0,0 \%$ & $100,0 \%$ & $0,0 \%$ & $0,0 \%$ \\
\hline & Bebidas & 33 & $n$ & 0 & 33 & 0 & 0 & 33 & 0 & 0 \\
\hline & edulcoradas & & $\%$ & $0,0 \%$ & $100,0 \%$ & $0,0 \%$ & $0,0 \%$ & $100,0 \%$ & $0,0 \%$ & $0,0 \%$ \\
\hline & & 42 & $\mathrm{n}$ & 9 & 33 & 0 & 1 & 41 & 0 & 0 \\
\hline $10 t a 1$ & & & $\%$ & $21,4 \%$ & $78,6 \%$ & $0,0 \%$ & $2,4 \%$ & $97,6 \%$ & $0,0 \%$ & $0,0 \%$ \\
\hline Comidas $\mathrm{p}$ & e-elaboradas & 11 & $\mathrm{n}$ & 0 & 11 & 0 & 1 & 8 & 2 & 0 \\
\hline & & & $\%$ & $0,0 \%$ & $100,0 \%$ & $0,0 \%$ & $9,1 \%$ & $72,7 \%$ & $18,2 \%$ & $0,0 \%$ \\
\hline Muestra T & & $\begin{array}{c}170 \\
3\end{array}$ & $\mathrm{n}$ & 144 & 346 & 1213 & 89 & 658 & 874 & 82 \\
\hline
\end{tabular}

* Alimentos que no pudieron clasificarse por falta de información.

Fuente: Elaboración Propia. 


\section{2- Discusión}

Numerosos países de la región han avanzado en el desarrollo de perfiles nutricionales para el diseño e implementación de la política de etiquetado frontal. Argentina aún no cuenta con un perfil de nutrientes, lo cual representa una barrera para la implementación de políticas alimentarias como sistemas de etiquetado frontal en los envases de alimentos $\mathrm{y}$ otras medidas fundamentales para mejorar los entornos alimentarios (entornos escolares, restricciones al marketing de alimentos altos en azúcar, grasas y sal, entre otras). Es fundamental que los perfiles de nutrientes se correspondan con los mensajes y con el modelo de patrón alimentario propuestos por las guías alimentarias en las regiones o países en los que se aplican ${ }^{8}$. Este es el primer estudio que evalúa el nivel de acuerdo de los perfiles nutricionales utilizados en políticas de etiquetado frontal con las GAPA.

Se evaluaron un total de seis perfiles latinoamericanos, el perfil de OPS, Uruguay, Chile y Perú, utilizados en sistemas de etiquetado frontal de advertencia, y el perfil de Ecuador y Bolivia utilizados en sistemas de semáforo. Todos los perfiles se aplican a los alimentos procesados y ultraprocesados sin hacer distinción entre categorías.

Los resultados indican que el perfil de OPS y el perfil de Uruguay presentan un acuerdo sustancial con las GAPA (OPS Gwet's AC1=0,663, Uruguay Gwet's $\mathrm{AC} 1=0,651)$, es decir, que permiten clasificar los grupos alimentarios de manera congruente con las recomendaciones nutricionales de salud pública. Clasifican "a promover" a los alimentos protectores como legumbres, cereales integrales, hortalizas feculentas, carnes, huevo, frutas, verduras, aceites, semillas, frutos secos, leche, y agua, la mayoría productos naturales o mínimamente procesados. Y clasifican "a limitar" a los alimentos de consumo opcional como bebidas azucaradas, galletitas, cereales de desayuno con azúcar, carnes procesados, pastas rellenas, entre otros. A excepción de ciertas categorías que son clasificadas como "a limitar" por dichos perfiles aún siendo promovidas por las GAPA como los quesos, yogures, y panes envasados.

Ambos perfiles nutricionales presentan resultados similares dado que el perfil de Uruguay se desarrolló en base al perfil de OPS estableciendo metas más permisivas como primer nivel de gradualidad para la implementación del etiquetado frontal ${ }^{6}$.

Las GAPA presentan mayor nivel de exigencia que los perfiles de Ecuador, Perú y Bolivia debido a que dichos perfiles presentan un bajo nivel de exigencia en los umbrales para todos los nutrientes. Esto se traduce en una menor cantidad de productos clasificados como "a limitar" en comparación con las GAPA. El menor nivel de 
exigencia de los perfiles frente a las GAPA se puede observar por ejemplo en la categoría de pastas rellenas (100\% GAPA vs 9,1\% el perfil de Perú y 45,5\% el perfil de Bolivia y Ecuador), carnes procesadas (100\% GAPA vs 76,9\% el perfil de Perú, 70,9\%, el perfil de Bolivia y 75,2\% el perfil de Ecuador), y en el grupo de alimentos de consumo opcional (100\% GAPA vs 56,5\% el perfil de Perú, 52,8\%, el perfil de Bolivia y $58 \%$ el perfil de Ecuador).

Por el contrario, las GAPA tienen un nivel de exigencia menor en comparación con el perfil de OPS, Chile y Uruguay debido a que dichos perfiles son los más exigentes en los umbrales de todos los nutrientes que incluyen, limitando una mayor cantidad de productos en comparación con las GAPA. El mayor nivel de exigencia de los perfiles frente a las GAPA se puede observar por ejemplo en el grupo de legumbres, cereales, papa, pan y pastas (GAPA $11,9 \%$ vs $58,9 \%$ perfil OPS, $80 \%$ perfil Chile y $40,5 \%$ perfil Uruguay), frutas y verduras (GAPA $24,5 \%$ vs $64,2 \%$ perfil OPS, 32,1\% perfil Chile y, $41,5 \%$ perfil Uruguay), y comidas, pre-elaboradas (GAPA 0\% vs $100 \%$ perfil OPS, 45,5\%, perfil Chile y, 90,9\% perfil Uruguay). Respecto al perfil de Chile, es importante mencionar que se posiciona como el segundo más exigente en la muestra total por ser el único que incluye umbral de energía, el cual determina que más de la mitad de los productos (57\%) se clasifiquen como "a limitar". Si se consideran el resto de los nutrientes, sin considerar energía, el segundo más exigente es el perfil de Uruguay. En cuanto al análisis de cada grupo de alimento, se observa que en el grupo Legumbres, cereales, papa, pan y pastas, las GAPA presentan un nivel de acuerdo discreto o menor con los perfiles nutricionales excepto el perfil de Perú que presenta un acuerdo sustancial. Todos los perfiles limitan a los alimentos principalmente por su alto contenido en sodio y/o en energía. El perfil de Chile es el que presenta menor acuerdo en este grupo (Gwet's $\mathrm{ACl}=-0,455)$ por ser el único que incluye un umbral máximo para energía lo cual determina que sea el más exigente ( $80 \%$ “a limitar”) especialmente en la categoría de cereales refinados (fideos, pastas frescas y secas) donde clasifica "a limitar" al 97,3\% de los productos principalmente por su alto valor energético. Por el contrario, el Perfil de Perú es el que presenta mayor acuerdo (Gwet's AC1=0,723) por presentar el umbral de sodio menos exigente.

En las categorías de panes, los perfiles descalifican no solo al pan blanco sino también al pan integral por su alto contenido en sodio. Este resultado no concuerda con la recomendación de consumo diario de las GAPA de cereales y derivados preferentemente integrales. Los perfiles que presentan mayor nivel de exigencia en esta 
categoría son el perfil de OPS (81,3\% pan blanco y 83,3\% pan integral) y el perfil de Chile $(95,8 \%$ pan blanco y $83,3 \%$ pan integral). Este resultado coincide con el estudio de Sudáfrica ${ }^{55}$ y el de Australia $^{51}$ dónde los perfiles evaluados limitan a los panes integrales por exceder el umbral de sodio mientras que las GABA promueven su consumo diario.

Si bien en Argentina existe una ley ${ }^{83}$ que regula el contenido de este nutriente crítico estableciendo valores máximos de $530 \mathrm{mg} \%$ para panes con salvado, y $501 \mathrm{mg} \%$ para panificados sin salvado, dicho contenido continúa siendo excesivo para la mayoría de los perfiles evaluados. Este resultado muestra la necesidad de disminuir las metas máximas establecidas en la ley para que las industrias de alimentos reformulen sus productos disminuyendo el contenido de sodio en panes.

En línea con la evidencia que demuestra los beneficios del consumo de frutas y verduras $^{84}$ todos los perfiles presentan un acuerdo sustancial o casi perfecto con las GAPA promoviendo su consumo, al igual que los estudios que validan el perfil del Reino Unido para Francia ${ }^{59}$ y Sudáfrica ${ }^{55}$. La única excepción es el perfil de OPS que al limitar a la mayoría de las verduras enlatadas por su alto contenido en sodio $(72 \%)$ resulta con menor acuerdo (Gwet's $\mathrm{AC} 1=0,218$ ). Las GAPA promueven su consumo en lata solo en el caso en que sea difícil obtenerlas en fresco.

En el grupo carnes y huevos, todos los perfiles nutricionales presentan un alto nivel de acuerdo con las GAPA (moderado o mayor). Coinciden en promover las carnes y huevos y en limitar la mayoría de las carnes procesadas como salchichas, hamburguesas, o nuggets de pollo por su alto contenido en sodio y grasas. En cuanto a las carnes enlatadas (en su mayoría atún en lata) los perfiles contradicen a las GAPA limitándolas por el contenido en sodio. El objetivo de las GAPA es no desalentar el consumo de pescado en las regiones con poco acceso al pescado fresco, privilegiando esta idea sobre el uso de enlatados con el posible aumento de consumo de sal. Estos resultados coinciden con el estudio que evalúa el perfil del Reino Unido para el uso de claims en Sudáfrica en donde el acuerdo para este grupo fue moderado, con acuerdo en las carnes procesadas y desacuerdo en los enlatados ${ }^{55}$.

En el grupo de Leche, yogur y quesos hay desacuerdo en los quesos para todos los perfiles con respecto a las GAPA que recomiendan su consumo en el mensaje $\mathrm{N}^{\circ} 6$ “Consumir diariamente leche, yogur o queso, preferentemente descremados". Las GAPA recomiendan la elección de quesos blandos antes que duros y aquellos que tengan menor contenido de grasas y sal. Sin embargo los perfiles descalifican a la 
mayoría de los quesos: limitan entre el 94,9\% y $100 \%$ de los quesos semiduros y duros y entre el $68,5 \%$ y $94,5 \%$ de lo quesos blandos por su alto contenido en sodio, grasas totales, grasas saturadas, grasas trans y/o energía. Algunas excepciones son la ricota y el queso untable descremado que resultan promovidos por el perfil de Chile y Perú, o un tipo de queso por salut promovido por el perfil de Uruguay. Resultados similares se encontraron en Francia ${ }^{59}$ y Australia ${ }^{51}$ por lo que decidieron adaptar el perfil para esta categoría. A través de esta medida se intenta promover el consumo de quesos por representar una fuente de calcio importante en la dieta de su población.

En cuanto los yogures, el perfil de OPS y el perfil de Uruguay clasifican "a limitar" a algunos yogures enteros por su alto contenido en grasas saturadas y el perfil de OPS limita todos los yogures parcialmente descremados por su alto contenido en sodio. Es importante mencionar que todos los yogures enteros presentan azúcares como ingrediente, sin embargo al no declarar su contenido en la tabla nutricional no han sido evaluados como nutriente crítico, lo cual supone que el acuerdo en este grupo resultaría aún más bajo siendo el yogur un producto recomendado por las guías alimentarias para su consumo diario.

En el grupo bebidas sin azúcar que incluye aguas, sodas, infusiones y bebidas edulcoradas, la mayoría de los perfiles acuerdan con las GAPA en promover su consumo, excepto el perfil de OPS y el de Uruguay que limitan productos en la categoría de bebidas edulcoradas por el alto contenido en sodio. Hay evidencia que demuestra el impacto negativo de los edulcorantes no calóricos en la salud ${ }^{85} 86$, por lo tanto las políticas alimentarias deberían promover el consumo de agua como reemplazo de las bebidas azucaradas. Sin embargo, tanto las GAPA como los perfiles no desmotivan su consumo, siendo el perfil de OPS el único que considera a los edulcorantes artificiales no calóricos como nutriente crítico.

Tanto en los yogures parcialmente descremados como en las bebidas edulcoradas, el perfil de OPS resulta el más exigente, aún sin haber considerado los edulcorantes, debido a que el límite de sodio se establece en relación al aporte calórico (1mg por caloría) lo cual determina un límite muy estricto en alimentos con muy bajo o nulo aporte calórico como es el caso de los yogures parcialmente descremados y las bebidas edulcoradas que presentan un promedio de $51,5 \mathrm{mg} \%$ y $18,5 \mathrm{mg} \%$ de sodio respectivamente.

En el caso del grupo de alimentos de consumo opcional las GAPA y los perfiles coinciden en limitar la mayoría de los productos con un alto nivel de acuerdo (moderado 
o mayor). La clasificación de este último grupo, conformado principalmente por alimentos ultraprocesados, se encuentra alineada con la evidencia que indica la necesidad de detener su consumo creciente a partir de regulaciones nacionales debido a su impacto negativo en la salud ${ }^{8788}$. Una revisión sistemática establece que este tipo de alimentos tiene una alta densidad energética, alto contenido en azúcar, sodio, grasas totales y grasas saturadas y un bajo contenido en micronutrientes y otros componentes bioactivos, y de fibra dietética ${ }^{89}$. Además, los productos ultraprocesados tienen características que fomentan su consumo en exceso y como snacks: se venden en tamaño grande y extra-grande, son hiper-apetecibles, tiene una vida útil larga, se transportan fácilmente, y se publicitan agresivamente ${ }^{20}$. La tendencia del patrón alimentario en Argentina en los últimos años (1996-2013) muestra un desplazamiento de la alimentación tradicional, basada en preparaciones caseras y alimentos frescos o mínimamente procesados por una dieta conformada cada vez más en alimentos ultraprocesados, lo cual se traduce en un deterioro en la calidad de la $\operatorname{dieta}^{90}$.

Este estudio presenta como principal fortaleza ser el primero que evalúa el nivel de acuerdo de los perfiles nutricionales regionales utilizados en políticas de etiquetado frontal con las GAPA con la metodología de validación propuesta por la OMS para adaptar perfiles ya existentes al contexto nacional ${ }^{8}$. Representa un insumo clave para el diseño e implementación de esta política en Argentina.

Una limitación del estudio es la utilización del rótulo nutricional de los productos como fuente de información del contenido nutricional sin comprobar por análisis de laboratorio. Además, como en Argentina la declaración de azúcares totales es opcional y no se declaran los azúcares libres, se consideró el contenido de carbohidratos declarado en el rótulo nutricional como equivalente al de azúcares totales en las categorías de bebidas azucaradas y de alimentos dulces (golosinas). El contenido de azúcares libres se calculó en toda la muestra a partir del dato de azúcares totales declarado según metodología propuesta por $\mathrm{OPS}^{19}$. Por último, no se evalúa la presencia de edulcorantes no calóricos, porque no se relevó esta variable en el total de la muestra, por lo cual se subestima el nivel de exigencia de los perfiles que lo incluyen como nutriente crítico. 


\section{3- Conclusiones}

Como conclusión, es importante desarrollar políticas basadas en evidencia científica para la prevención de las ECNT. Una de las políticas recomendadas por la OMS es la promoción de un etiquetado frontal en los alimentos para facilitar la elección de productos saludables en base a un perfil nutricional. Los resultados de este análisis muestran que todos los perfiles evaluados coinciden con las GAPA en limitar a la mayoría de los alimentos ultraprocesados con alto contenido en nutrientes críticos (sodio, grasas y azúcares) que causan las ECNT. Por el contrario, se encontró un menor nivel de acuerdo entre los perfiles y las GAPA en los alimentos protectores que las GAPA promueven su consumo diario o su consumo moderado por su aporte de nutrientes fundamentales para mantener un buen estado de salud.

Además, el estudio muestra que el perfil de OPS y el perfil de Uruguay serían los más adecuados de la región a ser utilizados en Argentina como insumo para una política de un etiquetado frontal a fin de promover las recomendaciones alimentarias $\mathrm{y}$ nutricionales.

Este estudio constituye un insumo clave para el desarrollo de un perfil nacional que sirva como herramienta en el diseño e implementación de una política de etiquetado frontal que guíe eficazmente a los argentinos hacia la elección de productos más saludables en línea con las recomendaciones de las GAPA. También sirve para promover otras políticas públicas para la prevención de ECNT como la regulación de las publicidades de alimentos no saludables dirigidas a niños; la promoción de entornos escolares saludables, y de políticas fiscales para orientar el consumo de alimentos, entre otras. Estas políticas van en línea con las recomendaciones emitidas por la OMS y OPS 7109192 . Se espera que la política de etiquetado frontal sea integral y se acompañe con una campaña de educación que tenga en cuenta el marco de una alimentación variada, amplia, inclusiva y saludable, para lograr un impacto favorable en el consumo de la población. 


\section{4- Bibliografía}

${ }^{1}$ Azais-Braesco V, Goffi C, Labouze E. Nutrient profiling: comparison and critical analysis of existing systems. Public health nutrition. 2006;9(05):613-22.

${ }^{2}$ Tetens I, Oberdörfer R, Madsen C, de Vries J. Nutritional characterisation of foods: Science-based approach to nutrient profiling. European journal of nutrition. 2007;46(2):4-14.

${ }^{3}$ World Health Organization. Regional Office for the Eastern Mediterranean. Nutrient profile model for the marketing of food and non-alcoholic beverages to children in the WHO Eastern Mediterranean Region. 2017. Disponible en: http://applications.emro.who.int/dsaf/EMROPUB 2017 en 19632.pdf Acceso 5 de Octubre 2018.

${ }^{4}$ Health Canada. Toward Front-of-Package Nutrition Labels for Canadians. Consultation Document. 2018. Disponible en: https://www.canada.ca/content/dam/canada/health-canada/migration/health-system-systemesante/consultations/labels-nutrition-etiquetage/alt/labels-nutrition-etiquetage-eng.pdf Acceso 5 de Octubre 2018.

${ }^{5}$ Ministerio de Salud de la Nación. Guías Alimentarias para la Población Argentina, Buenos Aires 2016. Disponible en: $\quad$ http://www.msal.gob.ar/images/stories/bes/graficos/0000000817cnt-201604 Guia Alimentaria completa web.pdf Acceso 5 de Octubre 2018

${ }^{6}$ Lake A, Townshend T. Obesogenic environments: exploring the built and food environments. The Journal of the Royal society for the Promotion of Health. 2006;126(6):262-7.

${ }^{7}$ Organización Mundial de la Salud. Informe de la Comisión para acabar con la obesidad infantil. 2016. Disponible en: http://apps.who.int/iris/bitstream/10665/206450/1/9789243510064 spa.pdf?ua=1 Acceso 5 de Octubre 2018.

${ }^{8}$ World Health Organization. Guiding principles and framework manual for the development or adaptation of nutrient profile models. Geneva: World Health Organization. Draft.

${ }_{9}^{9}$ Organización de las Naciones Unidas. Declaración Política de la Reunión de Alto Nivel de la Asamblea General sobre la Prevención y el Control de las Enfermedades No Transmisibles. 2011. Disponible en: http://www.un.org/es/comun/docs/?symbol=A/66/L Acceso 5 de Octubre 2018.

${ }^{10}$ OPS/FAO. Políticas y programas alimentarios para prevenir el sobrepeso y la obesidad. Lecciones aprendidas. 2018. Disponible en: http://www.fao.org/3/i8156es/I8156ES.pdf Acceso 5 de Octubre 2018.

${ }^{11}$ Tercera Encuesta Nacional de Factores de Riesgo para Enfermedades No Transmisibles. Ministerio de Salud de la Nación, Argentina; 2013.

${ }^{12}$ Encuesta Mundial de Salud Escolar - Argentina. Ministerio de Salud de la Nación, Argentina; 2012.

${ }^{13}$ Encuesta Nacional de Nutrición y Salud. Ministerio de Salud, Argentina, 2005.

${ }^{14}$ Daza, C. 2002. La obesidad: un desorden metabólico de alto riesgo para la salud. Colombia Médica, 33, 2. Disponible en: http://colombiamedica.univalle.edu.co/index.php/comedica/article/view/224/227 Acceso 5 de Octubre 2018

${ }^{15}$ Organización Mundial de la Salud, 2014. 10 datos sobre la obesidad. Disponible en: http://www.who.int/features/ factfiles/obesity/es/\# Acceso 5 de Octubre 2018.

${ }^{16}$ Withrow, D. Alter, D. A. 2011. The economic burden of obesity worldwide: a systematic review of the direct costs of obesity. Obesity reviews, 12(2), 131-141

${ }^{17}$ Clough, P., and Destremau, K. 2015. The Wider Economic and Social Costs of Obesity: A Discussion of the NonHealth Impacts of Obesity in New Zealand. Wellington: New Zealand Institute of Economic Research.

${ }^{18}$ Organización Mundial de la Salud. Serie de Informes Técnicos. Dieta, nutrición y prevención de enfermedades crónicas. 2003. Disponible en: http://apps.who.int/iris/bitstream/10665/42755/1/WHO_TRS_916_spa.pdf Acceso 5 de Octubre 2018.

${ }^{19}$ Dirección de Promoción de la Salud y Control de Enfermedades No Transmisibles. Boletín de Vigilancia de Enfermedades No Transmisibles y Factores de Riesgo Nro. 8. Obesidad: Determinantes, epidemiología y su abordaje desde la salud pública. . 2016. Disponible en: http://www.msal.gob.ar/ent/images/stories/vigilancia/pdf/2016-2 boletin-de-vigilancia-8.pdf Acceso 5 de Octubre 2018.

${ }^{20}$ OPS/OMS. Alimentos y bebidas ultraprocesados en América Latina: tendencias, efecto sobre la obesidad e implicaciones para las políticas públicas. Washington DC, 2015. Disponible en: http://iris.paho.org/xmlui/bitstream/handle/123456789/7698/9789275318645 esp.pdf Acceso 5 de Octubre 2018.

${ }^{21}$ Anand SS, Hawkes C, de Souza RJ, et al. Food Consumption and its Impact on Cardiovascular Disease: Importance of Solutions Focused on the Globalized Food System: A Report From the Workshop Convened by the World Heart Federation. J Am Coll Cardiol 2015; 66(14): 1590-614

${ }^{22}$ Monteiro CA, Moubarac JC, Cannon G, Ng SW, Popkin B. Ultra-processed products are becoming dominant in the global food system. Obesity Reviews 2013; 14: 21-8

${ }^{23}$ World Cancer Research Fund International. Curbing global sugar consumption: Effective food policy actions to help promote healthy diets and tackle obesity. 2015.

${ }^{24}$ World Health Organization. Guideline: Sugar intake for adults and children. In: WHO Department of Nutrition for Health and Development (NHD), editor. Geneva: WHO; 2015.

${ }^{25}$ Monteiro C, Cannon G. El gran tema en nutrición y salud pública es el ultra-procesamiento de los alimentos. 2012

${ }^{26}$ World Health Organization. Nutrient Profiling. Report of a WHO/IASO Technical meeting, London, 2010.

${ }^{27}$ Organización Panamericana de la Salud. Modelo de perfil de nutrientes de la Organización Panamericana de la Salud. Washington, DC: $2016 . \quad$ OPS, Disponible en: http://iris.paho.org/xmlui/bitstream/handle/123456789/18622/9789275318737 spa.pdf?sequence=9\&isAllowed=y Acceso 5 de Octubre 2018. 
${ }^{28}$ WHO Regional Office for Europe nutrient profile model. WHO Regional Office for Europe. 2015. Disponible en: http://www.euro.who.int/en/health-topics/disease-prevention/nutrition/publications/2015/who-regional-office-foreurope-nutrient-profile-model-2015

${ }^{29}$ Hernandez, M et col. Sistemas de perfilado nutricional : bases metodológicas para su análisis. CESNI, 2018 Disponible en: http://www.cesni.org.ar/archivos/biblioteca/Sistemas-de-Perfilado-Nutricional-Hernandez-Digital.pdf Acceso 5 de Octubre 2018.

${ }^{30}$ Perfiles nutricionales: intencionalidad política versus impacto real en salud pública. Informe científico técnico FINUT. Fundación Iberoamericana de Nutrición; 2016. Disponible en: http://www.finut.org/wpcontent/uploads/2016/03/Perfiles_Nutricionales 18032016 conPortadas.pdf Acceso 5 de Octubre 2018.

${ }^{31}$ UNICEF. Análisis de regulaciones y prácticas para el etiquetado de alimentos y bebidas para niños y adolescentes en algunos países de América Latina (Argentina, Chile, Costa Rica y México) y recomendaciones para facilitar la

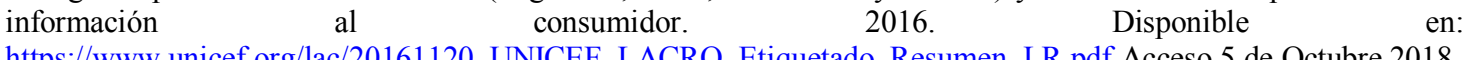
https://www.unicef.org/lac/20161120_UNICEF_LACRO_Etiquetado_Resumen_LR.pdf Acceso 5 de Octubre 2018.

${ }^{32}$ Hawley KL, Roberto CA, Bragg MĀ, Liu PJ, Schwartz MB, Brownell KD. The science on front-of-package food label. Public Health Nutrition 2013; 16: 430-439.

33 Codex Alimentarius. "Directrices sobre etiquetado nutricional. CAC/GL 2-1985. Disponible en: http://www.fao.org/fao-who-codexalimentarius/sh-

proxy/es/?lnk=1\&url=https\%253A\%252F\%252Fworkspace.fao.org\%252Fsites $\% 252 \mathrm{~F}$ codex $\% 252 \mathrm{FStandards} \% 252 \mathrm{~F}$ CAC\%2BGL\%2B2-1985\%252FCXG_002s.pdf Acceso 5 de Octubre 2018.

${ }^{34}$ Codex Alimentarius. E-Working Group. Guidelines on Front of pack nutrition labelling (FOPL) systems - CCFL. Disponible en: http://www.fao.org/fao-who-codexalimentarius/committees/ewg/detail/es/c/1062761/_Acceso 5 de Octubre 2018.

35 WCRF. Nourishing Framework. Nutrition Label standards and regulation on the use of claims on food. Disponible. 2018. en: https://www.wcrf.org/sites/default/files/Nutrition-labels.pdf Acceso 5 de Octubre 2018.

${ }^{36}$ Hersey JC, Wohlgenant KC, Arsenault JE, Kosa KM, Muth MK. Effects of front-of-package and shelf nutrition labeling systems on consumers. Nutrition reviews. 2013;71(1):1-14.

${ }^{37}$ Institute of Medicine (US) Committee on Examination of Front-of-Package Nutrition Rating Systems and Symbols. Front-of-Package Nutrition Rating Systems and Symbols: Phase I Report. Wartella EA, Lichtenstein AH, Boon CS, editors. Washington (DC): National Academies Press (US); 2010. Disponible en: http://www.ncbi.nlm.nih.gov/books/NBK209847/ Acceso 5 de Octubre 2018.

${ }_{38}$ Julia C, Péneau S, Buscail C, Gonzalez R, Touvier M, Hercberg S, et al. Perception of different formats of frontof-pack nutrition labels according to sociodemographic, lifestyle and dietary factors in a French population: crosssectional study among the NutriNet-Santé cohort participants. BMJ open. 2017;7(6):e016108.

${ }^{39}$ Corvalán C, Reyes M, Garmendia ML, Uauy R. Structural responses to the obesity and non-communicable diseases epidemic: the Chilean Law of Food Labeling and Advertising. Obesity reviews. 2013;14:79-87.

${ }^{40}$ Sacks G, Rayner M, Swinburn B. Impact of front-of-pack 'traffic-light'nutrition labelling on consumer food purchases in the UK. Health promotion international. 2009;24(4):344-52.

${ }^{41}$ Hodgkins C, Barnett J, Wasowicz-Kirylo G, Stysko-Kunkowska M, Gulcan Y, Kustepeli Y, et al. Understanding how consumers categorise nutritional labels: a consumer derived typology for front-of-pack nutrition labelling. Appetite. 2012;59(3):806-17.

42 Arrúa A, Machín L, Curutchet MR, Martínez J, Antúnez L, Alcaire F, Giménez A, Ares G. Warnings as directive front-of-pack nutrition labelling scheme: Comparison with the GDA and traffic light systems. Public Health Nutrition 2017; 19: 1-10.

43 Arrúa A, Curutchet MR, Rey N, Barreto P, Golovchenko N, Sellanes A, et al. Impact of front-of-pack nutrition information and label design on children's choice of two snack foods: Comparison of warnings and the traffic-light system. Appetite. 2017;116:139-46

44 World Health Organization. The Shake Technical Package for Salt Reduction. 2016. Disponible en: http://apps.who.int/iris/bitstream/10665/250135/1/9789241511346-eng.pdf?ua=1 Acceso 5 de Octubre 2018.

${ }^{45}$ Khandpur $\mathrm{N}$ et al. Are front-of-package warning labels more effective at communicating nutrition information than traffic-light labels? A randomized controlled experiment in a Brazilian sample. Nutrients, 10(6), 688, 2018.

46 FAO/OMS. Conferencia Internacional sobre Nutrición (CIN) 1992. Disponible en: http://www.fao.org/docrep/v7700t/v7700t04.htm Acceso 5 de Octubre 2018.

${ }^{47} \mathrm{FAO} / \mathrm{WHO}$. Preparation and use of food-based dietary guidelines. WHO Geneva; 1996. Disponible en: http://apps. who.int/iris/bitstream/handle/10665/42051/WHO TRS 880.pdf;jsessionid=044CD63B7504DE7F26C1 B5DDDF74C080?sequence=1 Acceso 5 de Octubre 2018.

${ }^{48} \mathrm{FAO} /$ WHO. Food-Based Dietary Guidelines. Disponible en: http://www.fao.org/nutrition/education/food-dietaryguidelines/background/en/ Acceso 5 de Octubre 2018.

${ }^{49}$ Monteiro CA, Cannon G, Moubarac J-C, Levy RB, Louzada MLC, Jaime PC. The UN Decade of Nutrition, the NOVA food classification and the trouble with ultra-processing. Public health nutrition. 2018;21(1):5-17.

${ }^{50}$ World Health Organization. Nutrient Profiling. Disponible : http://www.who.int/nutrition/topics/profiling/en/ Acceso 5 de Octubre 2018.

${ }^{51}$ Jones A, Rådholm K, Neal B. Defining 'Unhealthy’: A Systematic Analysis of Alignment between the Australian Dietary Guidelines and the Health Star Rating System. Nutrients. 2018;10(4):501.

52 Arambepola C, Scarborough P, Rayner M. Validating a nutrient profile model. Public health nutrition. 2008;11(4):371-8. 
${ }^{53}$ Rayner, M., Scarborough, P., and Lobstein, The UK Ofcom Nutrient Profiling Model - Defining 'healthy' and 'unhealthy' food and drinks for TV advertising to children. Disponible en: http://www.dph.ox.ac.uk/bhfhprg/publicationsandreports/acad-

publications/bhfhprgpublished/nutrientprofilemodel.Date Acceso 5 de Octubre 2018.

${ }^{54}$ Food Standards Australia New Zealand.Overview of the nutrient profiling scoring criterion. 2016. Disponible en: http://www.foodstandards.gov.au/industry/labelling/Pages/Consumerguide-to-NPSC.aspx. Acceso 5 de Octubre 2018

${ }^{55}$ Wicks M. The validation of a suitable nutrient profiling model for South Africa, North-West University. 2012. Disponible en: https://dspace.nwu.ac.za/handle/10394/9253 Acceso 5 de Octubre 2018.

56 Department of Health, Australia. Health star rating system. 2014. Disponible en: http://healthstarrating.gov.au/internet/healthstarrating/publishing.nsf/content/home.

${ }^{57}$ Julia C, Hercberg S. Nutriscore: evidence of the effectiveness of the French front-of-pack nutrition label. Ernahrungs Umschau. ; 64(12): p. 181-187

${ }^{58}$ Wentzel-Viljoen E. Nutrient profiling to develop a model for regulating health and nutrient claims: Experiences and lessons learned from South Africa Edelweiss Centre of Excellence for Nutrition North-West University South Africa. $2013 . \quad$ Disponible http://www.who.int/nutrition/topics/seminar 19Sept2013 GrandaSpain WentzelViljoen presentation.pdf?ua=1 Acceso 5 de Octubre 2018.

${ }^{59}$ Julia C, Kesse-Guyot E, Touvier M, Méjean C, Fezeu L, Hercberg S. Application of the British Food Standards Agency nutrient profiling system in a French food composition database. British Journal of Nutrition. 2014;112(10):1699-705.

${ }^{60}$ Julia C \& Hercberg S (2016) Research and lobbying conflicting on the issue of a front-of-pack nutrition labelling in France. Arch Public Health 74, 51.

${ }^{61}$ Julia C, Ducrot P, Peneau S, Deschamps V, Méjean C, Fézeu L, Touvier M, Hercberg S, Kesse-Guyot E. Discriminating nutritional quality of foods using the 5-Color nutrition label in the French food market: consistency with nutritional recommendations. Nutr J 2015;14:100.

${ }^{62}$ Food regulation. Front-of-pack labelling committee and working group meetings. 2012-2013. Disponible en: http://foodregulation.gov.au/internet/fr/publishing.nsf/Content/frontofpackcommittee Acceso 5 de Octubre 2018.

${ }^{63}$ Two year progress review report on the implementation of the Health Star Rating system. 2014-2016. Health Star

Rating Advisory Commmittee. Abril 2017. Disponible en
http://www.healthstarrating.gov.au/internet/healthstarrating/publishing.nsf/Content/145A3C2B51C5A741CA25803 D0006D3B4/\$File/Health-Star-Rating-Progress-Review-Report.pdf Acceso 5 de Octubre 2018.

${ }^{64}$ Foods Standards Agency Australia New Zealand. Short guide for industry to the Nutrient Profiling Scoring Criterion (NPSC) in standard 1.2.7 Nutrition,Health and related claims. Disponible en: http://www.foodstandards.gov.au/industry/abelling/Pages/Short-guide-for-industry-to-the-NPSC.aspx. Acceso 5 de Octubre 2018.

${ }^{65}$ Munene L-AE, Dumais L, Esslinger K, Jones-Mclean E, Mansfield E, Verreault M-F, et al. A surveillance tool to assess diets according to Eating Well with Canada's Food Guide. Health reports. 2015;26(11):12.

${ }^{66}$ Bend DVD, Dieren JV, Marques MDV, Wezenbeek NL, Kostareli N, Rodrigues PG, et al. A Simple Visual Model to Compare Existing Front-of-pack Nutrient Profiling Schemes. 2014.

${ }^{67}$ Universidad de Lujan. ARGENFOODS. Disponible en: http://www.argenfoods.unlu.edu.ar/ Acceso 5 de Octubre 2018.

${ }^{68}$ Allemandi L, Tiscornia MV, Ponce M, Castronuovo L, Dunford E, Schoj V. Sodium content in processed foods in Argentina: compliance with the national law. Cardiovascular diagnosis and therapy. 2015;5(3):197.

${ }^{69}$ Dunford E, Webster J, Metzler AB, Czernichow S, Mhurchu CN, Wolmarans P, et al. International collaborative project to compare and monitor the nutritional composition of processed foods. European journal of preventive cardiology. 2012;19(6):1326-32.

${ }^{70}$ Allemandi L, Castronuovo L, Tiscornia MV, Ponce M, Schoj V. Food advertising on Argentinean television: are ultra-processed foods in the lead? Public health nutrition. 2018;21(1):238-46.

${ }^{71}$ Fundación Interamericana del Corazón Argentina. Técnicas de marketing dirigidas a niños y niñas en envases de alimentos procesados de Argentina. 2017. Disponible en: http://www.ficargentina.org/wpcontent/uploads/2017/11/1708_informe_envases.pdf Acceso 5 de Octubre 2018.

${ }^{72}$ Rodrigues V, Rayner M, Fernandes A, de Oliveira R, Proença R, Fiates G. Nutritional quality of packaged foods targeted at children in Brazil: which ones should be eligible to bear nutrient claims? International Journal of Obesity. 2017;41(1):71.

${ }^{73}$ Rayner M, Scarborough P, Kaur A. Nutrient profiling and the regulation of marketing to children. Possibilities and pitfalls. Appetite. 2013;62:232-5.

${ }^{74}$ Scarborough P, Payne C, Agu C, Kaur A, Mizdrak A, Rayner M, et al. How important is the choice of the nutrient profile model used to regulate broadcast advertising of foods to children? A comparison using a targeted data set. European journal of clinical nutrition. 2013;67(8):815

${ }^{75}$ Wicks M, Wright H, Wentzel-Viljoen E. Restricting the marketing of foods and non-alcoholic beverages to children in South Africa: are all nutrient profiling models the same? British Journal of Nutrition. 2016;116(12):21509.

${ }^{76}$ Labonté M-È, Poon T, Mulligan C, Bernstein JT, Franco-Arellano B, L'Abbé MR. Comparison of global nutrient profiling systems for restricting the commercial marketing of foods and beverages of low nutritional quality to children in Canada. The American journal of clinical nutrition. 2017;106(6):1471-81. 
77 Cohen J. A coefficient of agreement for nominal scales. Educational and psychological measurement. 1960;20(1):37-46.

${ }^{78}$ Gwet KL. Computing inter-rater reliability and its variance in the presence of high agreement. British Journal of Mathematical and Statistical Psychology. 2008;61(1):29-48.

${ }^{79}$ Wongpakaran N, Wongpakaran T, Gwet KL. A comparison of Cohen's Kappa and Gwet's AC1 when calculating inter-rater reliability coefficients: a study conducted with personality disorder samples. BMC medical research methodology. 2013;13(1):61.

${ }^{80}$ Feinstein AR, Cicchetti DV. High agreement but low kappa: I. The problems of two paradoxes. Journal of clinical epidemiology. 1990;43(6):543-9.

${ }^{81}$ Gwet, K. (2014). Handbook of Inter-Rater Reliability, Advanced Analytics Press, Maryland, USA. ISBN: 9 780970806284.

${ }^{82}$ Landis JR, Koch GG. The measurement of observer agreement for categorical data. biometrics. 1977:159-74

${ }^{83} \mathrm{Li}$ M, Fan Y, Zhang X, Hou W, Tang Z. Fruit and vegetable intake and risk of type 2 diabetes mellitus: metaanalysis of prospective cohort studies. BMJ Open. 2014;4(11).

${ }^{84}$ Wang PY, Fang JC, Gao ZH, Zhang C, Xie SY. Higher intake of fruits, vegetables or their fiber reduces the risk of type 2 diabetes: A meta-analysis. Journal of diabetes investigation. 2016;7(1):56-69.

${ }^{85}$ McSweeney PL. Cheese problems solved: Elsevier; 2007.

${ }^{86}$ Suez J, Korem T, Zeevi D, Zilberman-Schapira G, Thaiss CA, Maza O, et al. Artificial sweeteners induce glucose intolerance by altering the gut microbiota. Nature. 2014;514(7521):181.

${ }^{87}$ Ruanpeng D, Thongprayoon C, Cheungpasitporn W, Harindhanavudhi T. Sugar and artificially sweetened beverages linked to obesity: a systematic review and meta-analysis. QJM: An International Journal of Medicine. 2017;110(8):513-20.

${ }^{88}$ Louzada MLC, Martins APB, Canella DS, Baraldi LG, Levy RB, Claro RM et al. Ultraprocessed foods and the nutritional dietary profile in Brazil. Rev Saude Publica 2015; 49: 38.

${ }^{89}$ Moubarac JC, Parra DC, Cannon G, Monteiro CA. Food classification systems based on food processing: significance and implications for policies and actions: a systematic literature review and assessment. Curr Obes Rep 2014; 3: 256-272.

${ }^{90}$ CESNI. La mesa Argentina en las últimas décadas: cambios en el patrón de consumo de alimentos y nutrientes 1996-2013. 2016. Disponible en: http://www.cesni.org.ar/archivos/biblioteca/LA-MESA-ARGENTINA-EN-LASULTIMAS-DOS-DECADAS.pdf Acceso 5 de Octubre 2018.

${ }^{91}$ Organización Panamericana de la Salud. Plan de acción para la prevención de la obesidad en la niñez y la adolescencia; 2014. Disponible en: https://www.paho.org/hq/dmdocuments/2015/Obesity-Plan-Of-Action-Child-Spa2015.pdf Acceso 5 de Octubre 2018.

92 World Health Organization. Global action plan for the prevention and control of noncommunicable diseases 20132020; 2013. Disponible en: https://www.who.int/nmh/events/ncd_action_plan/en/ Acceso 5 de Octubre 2018. 